\title{
19. BIOGENIC SEDIMENTATION AT SITE 847, EASTERN EQUATORIAL PACIFIC OCEAN, DURING THE PAST 3 M.Y. ${ }^{1}$
}

\author{
D.W. Murray, ${ }^{2}$ J.W. Farrell, ${ }^{2,3}$ and V. McKenna ${ }^{2}$
}

\begin{abstract}
Site 847 , located on the equator beneath the region of divergent-driven upwelling in the eastern tropical Pacific Ocean, provides a continuous record of biogenic sedimentation spanning the past $3 \mathrm{~m} . \mathrm{y}$. Sediments at the site are primarily composed of carbonate and opal microfossils, with secondary amounts of detrital material that was transported to the site by means of winds. The cyclic changes in the relative abundance of carbonate and opal during the past $1 \mathrm{~m} . \mathrm{y}$. exhibit a strong 100-k.y. variability, which generally corresponds to late Pleistocene climatic oscillations. A distinct, carbonate-poor interval is evident from the last major interglacial and has been attributed to a decrease in production rather than to dissolution. The long-term changes in $\mathrm{CaCO}_{3}$ mass accumulation rates (MARs) also are driven by production rather than dissolution. High rates near $2.8 \mathrm{Ma}$ are followed by a distinct minima near $1.8 \mathrm{Ma}$. $\mathrm{CaCO}_{3}$ MARs increase to a maxima near $0.4 \mathrm{Ma}$, followed by the decrease in production that occurred during the last interglacial period. Opal accumulations exhibit distinct maxima between 1.7 and $2.0 \mathrm{Ma}, 1.1$ and $1.3 \mathrm{Ma}$, and during glacial stages 6 and 2 . These increases by more than a factor of 2 in opal accumulation tentatively have been attributed to opal production.

Site 847 provides the eastern anchor of three sites studied from the central (near $140^{\circ} \mathrm{W}$ ) to eastern (near $95^{\circ} \mathrm{W}$ ) equatorial Pacific Ocean to evaluate spatial changes in carbonate and opal sedimentation in an area of strong divergence during the past 3 m.y. Modern oceanographic studies show a west to east gradient in surface productivity across this region. Analysis of the carbonate sedimentation for the past $3 \mathrm{~m} . \mathrm{y}$. and the opal sedimentation for the past $1 \mathrm{~m} . \mathrm{y}$. at the three sites reveals distinct differences in the respective gradients of accumulation. The west to east gradient in carbonate accumulation is small despite the fact that the eastern site is shallower and has better carbonate preservation. However, the gradient in opal accumulation is steep and shows a four- to fivefold increase from the central to the eastern equatorial Pacific sites. Thus, the surface gradient in primary production is reflected by bulk opal accumulation, not by carbonate accumulation.
\end{abstract}

\section{INTRODUCTION}

The eastern equatorial Pacific Ocean is an area of high biological productivity fueled by the divergence-driven upwelling of nutrientrich waters associated with the regional trade-wind systems. Chavez and Barber (1987) indicated that this region is responsible for as much as $50 \%$ of the global "new" production. An understanding of the response of this biological production to perturbations in climate will provide insights about the role of this area in the transfer of carbon from the surface waters to the deep sea over geologic time. Long continuous records of sediment deposition obtained from the eastern equatorial Pacific during Ocean Drilling Program (ODP) Leg 138 offer the potential to unravel the response of this area to major regional tectonic events (the closing of the Panamanian seaway) and global climate changes that occurred during the late Neogene (Mayer, Pisias, Janecek, et al, 1992).

Sediments recovered at equatorial sites during Leg 138 are largely a mixture of biogenic calcite and opal. Carbonate and opal shells of microplankton that accumulate beneath the equatorial upwelling zone provide an opportunity to quantify past changes in surface production. However, differential preservation of calcite and opal complicates the relationship between production of these microfossils and their accumulation in deep-sea sediments. Careful site selection was necessary to minimize these potential biases when interpreting the geological record. Calcite preservation is dependent upon the carbonate and organic carbon rain, water depth, and carbonate ion concentrations in the water column and pore waters (see Broecker and Peng, 1982; Archer,

${ }^{1}$ Pisias, N.G., Mayer, L.A., Janecek, T.R., Palmer-Julson, A., and van Andel, T.H. (Eds.), 1995. Proc. ODP, Sci. Results, 138: College Station, TX (Ocean Drilling Program). ${ }^{2}$ Department of Geological Sciences, Brown University, Providence, RI 02912. U.S.A.

${ }^{3}$ Present address: Department of Oceanography, University of British Columbia, Vancouver, B.C., Canada, V6T 1 Z4. 1991a, 1991b, and references therein). The relationships between production and accumulation are more reliable at shallow sites that exhibit little depth-dependent dissolution. Seawater is undersaturated with respect to opal at all depths of the ocean; therefore, only a small portion $(<10 \%)$ of the original rain has been preserved beneath the zone of equatorial upwelling (Broecker and Peng, 1982; Lyle et al., 1988; Archer et al., 1993). Broecker and Peng (1982) suggested that opal preservation is enhanced during periods with high rain rates. Archer et al. (1993) questioned this relationship and suggested that opal preservation in tropical regions reflects the solubility and dissolution kinetics of the opal raining to the seafloor rather than production. Despite this claim, opal concentration does exhibit a match to the global distribution of surface productivity (Leinen et al., 1986), the latitudinal changes in productivity in the eastern Pacific Ocean (Murray and Leinen, 1993), and is a reliable monitor of monsoonal upwelling in the Arabian Sea (Clemens et al., 1991). Although the relationship between opal production and preservation may be complex, sufficient evidence exists to suggest that opal accumulation in some regions at least may be a qualitative measure of surface production.

The goal of this study is to quantify the accumulation of carbonate and opal in a longitudinal transect along the equator so that spatial and temporal changes in surface production can be evaluated for this region during the late Pliocene to Pleistocene. This time interval includes the initiation of Northern Hemisphere glaciation, but it is after the closing of the Isthmus of Panama (Keigwin, 1982). Thus, the regional current patterns that are evident today (Fig. 1) are likely to have existed throughout the time interval considered. Changes in biogenic sediment accumulation should reflect regional to global perturbations in climate and oceanic properties. This study focuses on results from Site 847 $\left(0^{\circ} 11.6^{\prime} \mathrm{N}, 95^{\circ} 19.2^{\prime} \mathrm{W}\right.$, water depth of $\left.3334 \mathrm{~m}\right)$, which is the eastern anchor of a transect along the equator beginning in the central Pacific near $140^{\circ} \mathrm{W}$ (Fig. 1). We use data from the studies of Lyle et al. (1988), Farrell and Prell (1991), and Rea et al. (1991) for the area near $140^{\circ} \mathrm{W}$, and Farrell (1991) and Farrell and Prell (1991) for the middle location, Deep Sea Drilling Project (DSDP) Site 572 near $114^{\circ} \mathrm{W}$. 


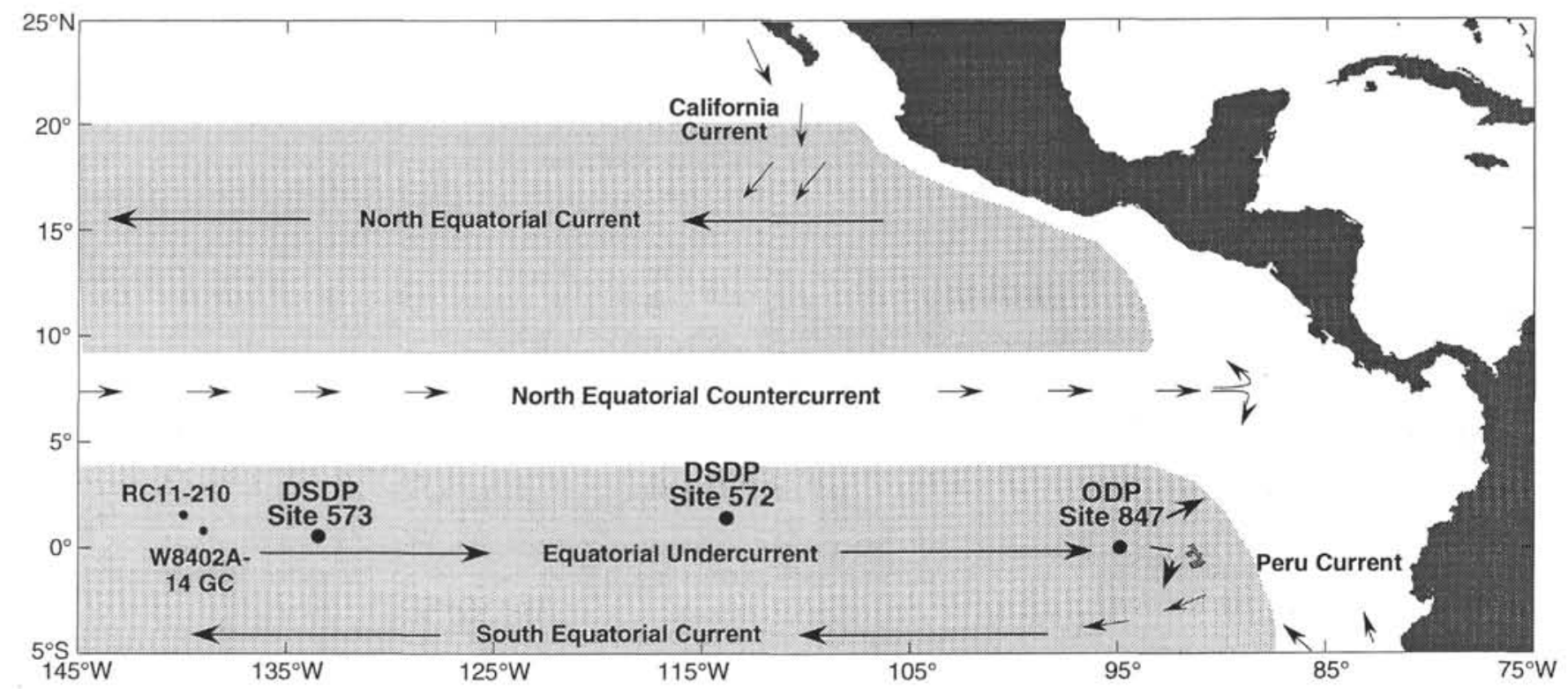

Figure 1. Location map of sites used in this study within the major regional current system. The shaded areas are the annual extent of the North and South Equatorial Current systems.

\section{METHODS}

The mean sedimentation rate for Site 847 over the past 3 m.y. is near $35 \mathrm{~m} / \mathrm{m}$.y. Samples were taken at $15-\mathrm{cm}$ intervals in Cores $138-847 \mathrm{C}-1 \mathrm{H}$ to $-9 \mathrm{H}$, which provide a temporal spacing close enough to resolve precessional-scale variability ( 23 k.y.) in biogenic sedimentation (Table 1). Additional samples were taken in discrete sections from Holes 847B and 847D to account for disturbed or missing sections that were recognized in Hole $847 \mathrm{C}$ (see below). Companion samples were taken at these same depths for foraminiferal and stable isotope studies, and the washing and counting procedures along with faunal and stable isotope data for the top $1 \mathrm{~m} . \mathrm{y}$. are presented in chapters by Farrell et al. (this volume) and McKenna et al. (this volume). Included in Table 2 are the dry weight percentage of the sample that is $>150-\mu \mathrm{m}$, as well as the percentage of whole foraminifers [100 $\times$ whole planktonic foraminifers/(whole planktonic foraminifers + fragments)] and the percentage of radiolarians [100 $\times$ radiolarians/(whole planktonic foraminifers + radiolarians)] in the $>150-\mu \mathrm{m}$ portion of selected samples.

\section{Composite Depth Section}

One of the first tasks in a study of $10^{4}$ - to $10^{5}$-yr scale of variability from deep-sea cores is to ensure that the stratigraphic section reflects a continuous record of sediment deposition spanning the period of interest. The existence of sediment recovery gaps between successive cores using the advanced hydraulic piston corer (APC) from DSDP and ODP sites is well documented (Ruddiman et al., 1989; Robinson, 1990; Murray and Prell, 1991; Farrell and Janecek, 1991). These missing sections are evident even with consistent recovery of more than $9.5 \mathrm{~m}$ for each $9.5 \mathrm{~m}$ drilled, which indicates that the cored material is somehow expanded in the recovery processes. Multiple offset holes drilled at a single site provide a means to construct a continuous stratigraphic section as material from adjacent holes can be "spliced" together to construct a continuous depth section for the site. One of the primary objectives of the Leg 138 Shipboard Scientific Party was to construct composite depth sections for each of the sites drilled (Hagelberg et al., 1992). Each composite depth section considered the stratigraphies from all holes at a site to create a continuous section. Cores from adjacent holes were moved relative to one another by a fixed amount to provide a high interhole correlation for gamma-ray attenuation porosity evaluator (GRAPE), magnetic susceptibility, and color reflectance data. These data were measured in each core soon after recovery at a sample spacing of approximately 2 to $5 \mathrm{~cm}$. The composite stratigraphic section for Site 847, based on the shipboard work, consists of cored intervals from Holes $847 \mathrm{~B}$, $847 \mathrm{C}$, and $847 \mathrm{D}$ (Hagelberg et al., this volume). Samples used in this study came primarily from Hole 847 C, with splices from Holes 847B and $847 \mathrm{D}$. Thus, only a portion of the samples were taken from the sections included in the shipboard spliced composite record. Because significant stretching and squeezing of sections is apparent among the holes at a given site (Hagelberg et al., this volume), a simple transfer of the shipboard meters composite depth (mcd) to Hole $847 \mathrm{C}$ will yield offsets in the structure of Hole $847 \mathrm{C}$ data relative to other holes and the composite. As shown in Figure 2A, GRAPE data in Holes $847 \mathrm{C}$ and $847 \mathrm{D}$ can be offset by as much as $0.5 \mathrm{~m}$; the GRAPE maxima at points $\mathrm{A}, \mathrm{B}$, and $\mathrm{C}$ for Hole $847 \mathrm{C}$ are offset in composite depth from the same events in Hole 847D labeled $\mathrm{A}^{\prime}, \mathrm{B}^{\prime}$, and $\mathrm{C}^{\prime}$.

A revision is needed to account for the offsets that arise when shipboard mcd is assigned to sections other than those used in the composite. Hagelberg et al. (this volume) derived a revised meters composite depth (rmcd) scale, which stretched and squeezed the composite depth sections within each core to align structure in GRAPE records among holes (Fig. 2B). However, this rmcd (Table 1) assumes a common GRAPE structure for a site and adjusts depths in each hole to fit that structure. For the 579 sample intervals from Hole $847 \mathrm{C}$, which measured approximately $15 \mathrm{~cm}$ in ODP depth, 98 were stretched or squeezed by at least $5 \mathrm{~cm}$ (Fig. 3). In the extreme case, a $15-\mathrm{cm}$ sample interval within one core, between Samples 138-847C-1H-1, 77 $\mathrm{cm}$, and $-1 \mathrm{H}-1,92 \mathrm{~cm}$, was stretched by $34 \mathrm{~cm}$ using this method. Sedimentation rates derived from the adjusted depth scale may not reflect the true sedimentation rate for an interval within a given hole. Because we develop an isotope stratigraphy from measurements on samples from Holes $847 \mathrm{C}$ and $847 \mathrm{D}$, including sections other than the ones used in the final composite for the site, and we use this chronostratigraphy in calculating sedimentation rates and mass accumulation rates (MARs) for each sample, the rmcd scale does not meet the needs of our project. Therefore, a composite depth stratigraphy for Cores $1 \mathrm{H}$ to $9 \mathrm{H}$ ( $\sim 0$ to $3 \mathrm{Ma}$ ) was developed for Site 847 by selecting Hole $847 \mathrm{C}$ as the primary record and using sections from Holes $847 \mathrm{~B}$ and $847 \mathrm{D}$ to fill in coring gaps or disturbed intervals. This composite depth reconstruction for Site 847 is summarized in Figure 4 and Table 3. We 

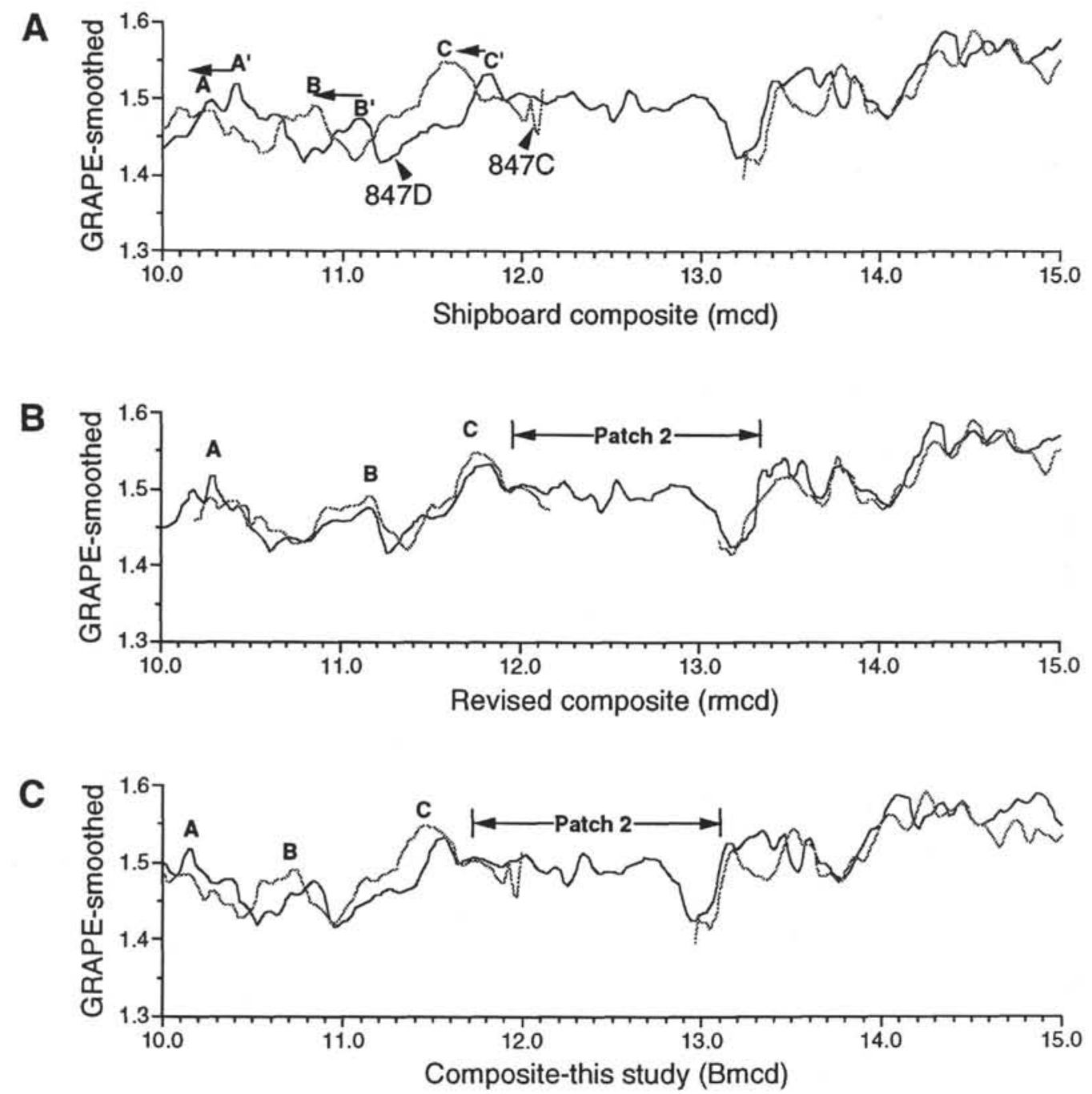

Figure 2. Composite depth reconstruction using smoothed (nine-point Gaussian filter) GRAPE data from Holes $847 \mathrm{C}$ and 847D between 10 and $15 \mathrm{~m}$. A. Shipboard composite depths (med) and applied to data from Holes 847C and 847D. Note offsets in GRAPE structure at events A-A', B-B', and C-C'. B. Revised composite depth for Site 847 after Hagelberg et al. (this volume). C. Meters composite depth (Bmcd) constructed for this study. The reconstruction is similar to that in Figure 2A, but choosing Hole $847 \mathrm{C}$ as the primary record and patches and core breaks coming mostly from Hole 847D.

use the notation "Bmcd" (Brown mcd) to distinguish these depths from other composite reconstructions. The new stratigraphy has the same limitations noted above for the shipboard mod when using an alternative hole as the primary record (Fig. 2C). Care was taken to ensure that GRAPE events adjacent to the patches were aligned to avoid replication and to ensure representation of these events in the composite section.

\section{Chronostratigraphy and Sedimentation Rates}

Shackleton et al. (1992) showed that GRAPE density records can be correlated among the Leg 138 sites and that these cycles have a high correlation to solar insolation variations. Shackleton et al. (this volume) use this correlation to assign a chronostratigraphy to the GRAPE records by matching insolation maxima of known age with GRAPE maxima. A total of 89 events between 0 and $3 \mathrm{Ma}$ were used in the age model for Site 847 (Table 4). The ages based on Shackleton et al.'s (this volume) chronology were assigned to samples in this study (Table 1) through interpolation by assuming a linear sedimentation rate of rmcd between datums. Sedimentation rates were assigned to each sample by the rmcd and age difference between a sample and the adjacent younger sample:

$$
\text { Sedimentation } \operatorname{rate}_{(i)}=\left[\operatorname{Depth}_{(i)}-\operatorname{Depth}_{(i-1)}\right] /\left[\operatorname{Age}_{(i)}-\text { Age }_{(i-1)}\right] \text {. }
$$

Farrell et al. (this volume) provide an independent chronostratigraphy for Site 847 spanning the top 1.14 m.y., based on oxygen isotope stratigraphy for the composite depth section developed in this study, using the planktonic foraminifer Globigerinoides sacculifer (Table 1). A total of 58 events were used for this age model. Farrell et al. (this volume) discuss the differences between the oxygen isotope and GRAPE-based chronologies. Because the oxygen isotope age model is independent of the carbonate changes, we have used this chronology for our time-series analysis and the discussion of the glacial to interglacial variations over the past $1 \mathrm{~m} . \mathrm{y}$. Shackleton et al. (this volume) incorporated data from the oxygen isotope studies of Leg 138 sites and presents a revision of the GRAPE-based age model over the past 1 m.y., in addition to that derived by using GRAPE records alone.

\section{Chemical Data}

Calcium carbonate and opal concentrations were measured in 5$\mathrm{cm}^{3}$ samples of bulk sediment (Table 2). All samples were freeze-dried and two-thirds of the dried sample was removed and ground with a mortar and pestle. Before being analyzed, the ground samples were 
Table 1. Site 847 samples and corresponding depths, ages, and sedimentation rates.

\begin{tabular}{|c|c|c|c|c|c|c|c|c|}
\hline \multirow[b]{2}{*}{$\begin{array}{l}\text { Core, section, } \\
\text { interval (cm) }\end{array}$} & \multirow[b]{2}{*}{$\begin{array}{l}\text { ODP } \\
\text { depth } \\
\text { (mbsf) }\end{array}$} & \multirow{2}{*}{$\begin{array}{l}\text { Shipboard } \\
\text { composite } \\
\text { depth } \\
\text { (mcd) }\end{array}$} & Revised & Composite & $\mathrm{Ag}$ & & Sediment: & ion rates \\
\hline & & & $\begin{array}{l}\text { composite } \\
\text { depth } \\
\text { (rmcd) }\end{array}$ & $\begin{array}{l}\text { depth, } \\
\text { this study } \\
\text { (Bmed) }\end{array}$ & $\begin{array}{l}\text { Shackleton } \\
\text { age model } \\
\text { (Ma) }\end{array}$ & $\begin{array}{l}\text { Isotope } \\
\text { age model } \\
\text { (Ma) }\end{array}$ & $\begin{array}{c}\text { Shackleton } \\
\text { ages } \\
(\mathrm{m} / \mathrm{m} . \mathrm{y} .)\end{array}$ & $\begin{array}{c}\text { Isotope } \\
\text { ages } \\
(\mathrm{m} / \mathrm{m} . \mathrm{y} .)\end{array}$ \\
\hline 138-847B- & & & & & & & & \\
\hline $1 \mathrm{H}-1,2$ & $0.02^{2}$ & 0.02 & 0.02 & 0.02 & 0.0010 & 0.0060 & - & - \\
\hline IH-I.19 & 0.19 & 0.19 & 0.19 & 0.19 & 0.0096 & 0.0093 & 19.82 & 50.77 \\
\hline $1 \mathrm{H}-1.30$ & 0.30 & 0.30 & 0.30 & 0.30 & 0.0151 & 0.0115 & 19.82 & 50.77 \\
\hline IH-1.47 & 0.47 & 0.47 & 0.47 & 0.47 & 0.0237 & 0.0149 & 19.82 & 50.77 \\
\hline IH-I. 59 & 0.59 & 0.59 & 0.59 & 0.59 & 0.0298 & 0.0172 & 19.82 & 50.77 \\
\hline $1 \mathrm{H}-1.77$ & 0.77 & 0.77 & 0.77 & 0.77 & 0.0388 & 0.0205 & 19.82 & 55.46 \\
\hline $\mathrm{IH}-\mathrm{I}, 92$ & 0.92 & 0.92 & 0.92 & 0.92 & 0.0464 & 0.0229 & 19.82 & 61.11 \\
\hline $1 \mathrm{H}-\mathrm{I}, 107$ & 1.07 & 1.07 & 1.07 & 1.07 & 0.0540 & 0.0254 & 19.82 & 61.11 \\
\hline $\mathrm{IH}-\mathrm{I}, 123$ & 1.23 & 1.23 & 1.24 & 1.23 & 0.0579 & 0.0280 & 40.57 & 61.11 \\
\hline $\mathrm{IH}-1,137$ & 1.37 & 1.37 & 1.40 & 1.37 & 0.0602 & 0.0317 & 62.31 & 37.60 \\
\hline $\mathrm{IH}-2,2$ & 1.52 & 1.52 & 1.55 & 1.52 & 0.0626 & 0.0357 & 62.31 & 37.60 \\
\hline $\mathrm{IH}-2,24$ & 1.74 & 1.74 & 1.75 & 1.74 & 0.0661 & 0.0416 & 62.31 & 37.60 \\
\hline IH -2.37 & 1.87 & 1.87 & 1.87 & 1.87 & 0.0682 & 0.0450 & 62.31 & 37.60 \\
\hline $\mathrm{IH}-2,52$ & 2.02 & 2.02 & 2.02 & 2.02 & 0.0706 & 0.0490 & 62.31 & 37.60 \\
\hline $\mathrm{IH}-2.67$ & 2.17 & 2.17 & 2.17 & 2.17 & 0.0730 & 0.0530 & 62.31 & 37.60 \\
\hline $\mathrm{IH}-2,80$ & 2.30 & 2.30 & 2.30 & 2.30 & 0.0751 & 0.0592 & 62.31 & 20.83 \\
\hline $\mathrm{IH}-2,92$ & 2.42 & 2.42 & 2.42 & 2.42 & 0.0770 & 0.0650 & 62.31 & 20.83 \\
\hline 1H-2, 107 & 2.57 & 2.57 & 2.57 & 2.57 & 0.0794 & 0.0700 & 62.31 & 30.00 \\
\hline $\mathrm{IH}-2,123$ & 2.73 & 2.73 & 2.73 & 2.73 & 0.0820 & 0.0753 & 62.31 & 30.00 \\
\hline $1 \mathrm{H}-2,137$ & 2.87 & 2.87 & 2.87 & 2.87 & 0.0860 & 0.0800 & 35.24 & 30.00 \\
\hline $\mathrm{IH}-3,2$ & 3.02 & 3.02 & 3.02 & 3.02 & 0.0902 & 0.0855 & 35.24 & 27.50 \\
\hline $1 \mathrm{H}-3.17$ & 3.17 & 3.17 & 3.17 & 3.17 & 0.0945 & 0.0909 & 35.24 & 27.50 \\
\hline $\mathrm{IH}-3,30$ & 3.30 & 3.30 & 3.30 & 3.30 & 0.0982 & 0.0956 & 35.24 & 27.50 \\
\hline $1 \mathrm{H}-3,47$ & 3.47 & 3.47 & 3.47 & 3.47 & 0.1030 & 0.1018 & 35.24 & 27.50 \\
\hline $138-847 \mathrm{C}-$ & & & & & & & & \\
\hline $1 \mathrm{H}-1,122$ & 3.22 & 3.62 & 3.60 & 3.50 & 0.1042 & 0.1029 & 25.65 & 27.50 \\
\hline $1 \mathrm{H}-1,137$ & 3.37 & 3.77 & 3.74 & 3.65 & 0.1100 & 0.1084 & 25.65 & 27.50 \\
\hline $1 \mathrm{H}-2,2$ & 3.52 & 3.92 & 3.87 & 3.80 & 0.1159 & 0.1138 & 25.65 & 27.50 \\
\hline IH $-2,17$ & 3.67 & 4.07 & 4.00 & 3.95 & 0.1217 & 0.1193 & 25.65 & 27.50 \\
\hline $1 \mathrm{H}-2,32$ & 3.82 & 4.22 & 4.14 & 4.10 & 0.1271 & 0.1239 & 28.04 & 32.72 \\
\hline IH $-2,47$ & 3.97 & 4.37 & 4.30 & 4.25 & 0.1310 & 0.1276 & 37.72 & 40.38 \\
\hline $1 \mathrm{H}-2,65$ & 4.15 & 4.55 & 4.51 & 4.43 & 0.1358 & 0.1320 & 37.72 & 40.38 \\
\hline $1 \mathrm{H}-2,77$ & 4.27 & 4.67 & 4.67 & 4.55 & 0.1390 & 0.1350 & 37.72 & 40.38 \\
\hline $1 \mathrm{H}-2,92$ & 4.42 & 4.82 & 4.87 & 4.70 & 0.1430 & 0.1382 & 37.72 & 46.88 \\
\hline $1 \mathrm{H}-2,107$ & 4.57 & 4.97 & 5.05 & 4.85 & 0.1469 & 0.1414 & 37.72 & 46.88 \\
\hline $1 \mathrm{H}-2,122$ & 4.72 & 5.12 & 5.20 & 5.00 & 0.1509 & 0.1446 & 37.72 & 46.88 \\
\hline $1 \mathrm{H}-2,137$ & 4.87 & 5.27 & 5.32 & 5.15 & 0.1549 & 0.1478 & 37.72 & 46.88 \\
\hline $\mathrm{IH}-3,2$ & 5.02 & 5.42 & 5.44 & 5.30 & 0.1589 & 0.1510 & 37.72 & 46.88 \\
\hline IH $-3,17$ & 5.17 & 5.57 & 5.57 & 5.45 & 0.1629 & 0.1560 & 37.72 & 30.00 \\
\hline IH $-3,32$ & 5.32 & 5.72 & 5.71 & 5.60 & 0.1668 & 0.1610 & 37.72 & 30.00 \\
\hline IH $-3,47$ & 5.47 & 5.87 & 5.87 & 5.75 & 0.1708 & 0.1660 & 37.72 & 30.00 \\
\hline IH -3.62 & 5.62 & 6.02 & 6.03 & 5.90 & 0.1748 & 0.1710 & 37.72 & 30.00 \\
\hline $1 \mathrm{H}-3.77$ & 5.77 & 6.17 & 6.19 & 6.05 & 0.1788 & 0.1750 & 37,72 & 37.50 \\
\hline $1 \mathrm{H}-3,92$ & 5.92 & 6.32 & 6.34 & 6.20 & 0.1827 & 0.1790 & 37.72 & 37.50 \\
\hline $1 \mathrm{H}-3,107$ & 6.07 & 6.47 & 6.48 & 6.35 & 0.1867 & 0.1830 & 37.72 & 37.50 \\
\hline $1 \mathrm{H}-3,122$ & 6.22 & 6.62 & 6.61 & 6.50 & 0.1907 & 0.1861 & 37.72 & 47.73 \\
\hline $1 \mathrm{H}-3,137$ & 6.37 & 6.77 & 6.76 & 6.65 & 0.1947 & 0.1893 & 37.72 & 47.73 \\
\hline $1 \mathrm{H}-4.2$ & 6.52 & 6.92 & 6.91 & 6.80 & 0.1986 & 0.1924 & 37.72 & 47.73 \\
\hline $1 \mathrm{H}-4,17$ & 6.67 & 7.07 & 7.07 & 6.95 & 0.2026 & 0.1977 & 37.72 & 28.64 \\
\hline $\mathrm{IH}-4,32$ & 6.82 & 7.22 & 7.22 & 7.10 & 0.2066 & 0.2050 & 37.72 & 20.45 \\
\hline $1 \mathrm{H}-4.47$ & 6.97 & 7.37 & 7.36 & 7.25 & 0.2106 & 0.2160 & 37.72 & 13.64 \\
\hline $1 \mathrm{H}-4.62$ & 7.12 & 7.52 & 7.51 & 7.40 & 0.2146 & 0.2220 & 37.72 & 25.00 \\
\hline $1 \mathrm{H}-4.77$ & 7.27 & 7.67 & 7.67 & 7.55 & 0.2188 & 0.2280 & 35.14 & 25.00 \\
\hline $1 \mathrm{H}-4,92$ & 7.42 & 7.82 & 7.84 & 7.70 & 0.2250 & 0.2313 & 24.32 & 45.00 \\
\hline $\mathrm{IH}-4,107$ & 7.57 & 7.97 & 8.03 & 7.85 & 0.2312 & 0.2347 & 24.32 & 45.00 \\
\hline $1 \mathrm{H}-4,122$ & 7.72 & 8.12 & 8.20 & 8.00 & 0.2373 & 0.2380 & 24.32 & 45.00 \\
\hline $1 \mathrm{H}-4,137$ & 7.87 & 8.27 & 8.36 & 8.15 & 0.2435 & 0.2417 & 24.32 & 40.91 \\
\hline IH $-5,2$ & 8.02 & 8.42 & 8.52 & 8.30 & 0.2497 & 0.2453 & 24.32 & 40.91 \\
\hline IH $-5,17$ & 8.17 & 8.57 & 8.68 & 8.45 & 0.2558 & 0.2490 & 24.32 & 40.91 \\
\hline IH-5. 32 & 8.32 & 8.72 & 8.83 & 8.60 & 0.2620 & 0.2557 & 24.32 & 22.50 \\
\hline IH $-5,47$ & 8.47 & 8.87 & 8.98 & 8.75 & 0.2666 & 0.2623 & 32.68 & 22.50 \\
\hline IH $-5,62$ & 8.62 & 9.02 & 9.11 & 8.90 & 0.2712 & 0.2690 & 32.68 & 22.50 \\
\hline IH -5.77 & 8.77 & 9.17 & 9.24 & 9.05 & 0.2758 & 0.2730 & 32.68 & 37.50 \\
\hline IH -5.92 & 8.92 & 9.32 & 9.39 & 9.20 & 0.2804 & 0.2770 & 32.68 & 37.50 \\
\hline $1 \mathrm{H}-5,107$ & 9.07 & 9.47 & 9.56 & 9.35 & 0.2850 & 0.2810 & 32.68 & 37.50 \\
\hline $1 \mathrm{H}-5,122$ & 9.22 & 9.62 & 9.77 & 9.50 & 0.2895 & 0.2850 & 32.68 & 37.50 \\
\hline $1 \mathrm{H}-5,137$ & 9.37 & 9.77 & 9.97 & 9.65 & 0.2958 & 0.2894 & 23.79 & 34.09 \\
\hline IH $-6,2$ & 9.52 & 9.92 & 10.12 & 9.80 & 0.3023 & 0.2942 & 23.10 & 31.25 \\
\hline $1 \mathrm{H}-6.17$ & 9.67 & 10.07 & 10.25 & 9.95 & 0.3088 & 0.2990 & 23.10 & 31.25 \\
\hline $\mathrm{IH}-6,32$ & 9.82 & 10.22 & 10.37 & 10.10 & 0.3141 & 0.3030 & 28.26 & 37.50 \\
\hline iH $-6,47$ & 9.97 & 10.37 & 10.51 & 10.25 & 0.3189 & 0.3085 & 31.82 & 27.08 \\
\hline IH-6.62 & 10.12 & 10.52 & 10.70 & 10.40 & 0.3236 & 0.3141 & 31.82 & 27.08 \\
\hline $1 \mathrm{H}-6,77$ & 10.27 & 10.67 & 10.93 & 10.55 & 0.3283 & 0.3196 & 31.82 & 27.08 \\
\hline IH $-6,92$ & 10.42 & 10.82 & 11.13 & 10.70 & 0.3330 & 0.3252 & 31.82 & 27.08 \\
\hline $1 \mathrm{H}-6,107$ & 10.57 & 10.97 & 11.28 & 10.85 & 0.3368 & 0.3307 & 39.05 & 27.08 \\
\hline IH $-6,122$ & 10.72 & 11,12 & 11.41 & 11.00 & 0.3407 & 0.3362 & 39.05 & 27.08 \\
\hline $1 \mathrm{H}-6,137$ & 10.87 & 11.27 & 11.52 & 11.15 & 0.3445 & 0.3407 & 39.05 & 33.62 \\
\hline IH- 7,2 & 11.02 & 11.42 & 11.63 & 11.30 & 0.3484 & 0.3441 & 39.05 & 44.31 \\
\hline $1 \mathrm{H}-7.17$ & 11.17 & 11.57 & 11.75 & 11.45 & 0.3522 & 0.3475 & 39.05 & 44.31 \\
\hline $\mathrm{IH}-7,32$ & 11.32 & 11.72 & 11.88 & 11.60 & 0.3570 & 0.3508 & 31.30 & 44.31 \\
\hline IH-7.47 & 11.47 & 11.87 & 12.02 & 11.75 & 0.3626 & 0.3542 & 26.67 & 44.31 \\
\hline
\end{tabular}


Table 1 (continued).

\begin{tabular}{|c|c|c|c|c|c|c|c|c|}
\hline \multirow[b]{2}{*}{$\begin{array}{l}\text { Core, section, } \\
\text { interval }(\mathrm{cm})\end{array}$} & \multirow[b]{2}{*}{$\begin{array}{l}\text { ODP } \\
\text { depth } \\
\text { (mbsf) }\end{array}$} & \multirow{2}{*}{$\begin{array}{c}\text { Shipboard } \\
\text { composite } \\
\text { depth } \\
\text { (mcd) }\end{array}$} & \multirow{2}{*}{$\begin{array}{c}\text { Revised } \\
\text { composite } \\
\text { depth } \\
\text { (rmcd) }\end{array}$} & Composite & & & Sediment: & tion rates \\
\hline & & & & $\begin{array}{l}\text { depth, } \\
\text { this study } \\
\text { (Bmod) }\end{array}$ & $\begin{array}{c}\text { Shackleton } \\
\text { age model } \\
\text { (Ma) }\end{array}$ & $\begin{array}{c}\text { Isotope } \\
\text { age model } \\
(\mathrm{Ma})\end{array}$ & $\begin{array}{l}\text { Shackleton } \\
\text { ages } \\
(\mathrm{m} / \mathrm{m} \cdot \mathrm{y} .)\end{array}$ & $\begin{array}{c}\text { Isotope } \\
\text { ages } \\
(\mathrm{m} / \mathrm{m} . \mathrm{y} .)\end{array}$ \\
\hline 138-847D- & & & & & & & & \\
\hline $2 \mathrm{H}-3,62$ & 10.22 & 12.02 & 12.04 & 11.76 & 0.3630 & 0.3545 & 26.67 & 44.31 \\
\hline $2 \mathrm{H}-3,77$ & 10.37 & 12.17 & 12.16 & 11.91 & 0.3686 & 0.3578 & 26.67 & 44.31 \\
\hline $2 \mathrm{H}-3.92$ & 10.52 & 12.32 & 12.28 & 12.06 & 0.3734 & 0.3612 & 31.21 & 44.31 \\
\hline $2 \mathrm{H}-3,107$ & 10.67 & 12.47 & 12.41 & 12.21 & 0.3770 & 0.3646 & 41.94 & 44.31 \\
\hline $2 \mathrm{H}-3,122$ & 10.82 & 12.62 & 12.55 & 12.36 & 0.3806 & 0.3680 & 41.94 & 44.31 \\
\hline $2 \mathrm{H}-3.137$ & 10.97 & 12.77 & 12.68 & 12.51 & 0.3842 & 0.3750 & 41.94 & 21.43 \\
\hline $2 \mathrm{H}-4,2$ & 11.12 & 12.92 & 12.82 & 12.66 & 0.3877 & 0.3802 & 41.94 & 29.00 \\
\hline $2 \mathrm{H}-4.17$ & 11.27 & 13.07 & 13.00 & 12.81 & 0.3913 & 0.3853 & 41.94 & 29.00 \\
\hline $2 \mathrm{H}-4,32$ & 11.42 & 13.22 & 13.20 & 12.96 & 0.3949 & 0.3905 & 41.94 & 29.00 \\
\hline $138-847 \mathrm{C}-$ & & & & & & & & \\
\hline $2 \mathrm{H}-\mathrm{I}, 17$ & 11.67 & 13.35 & 13.23 & 13.08 & 0.3977 & 0.3947 & 41.94 & 29.00 \\
\hline $2 \mathrm{H}-1,30$ & 11.82 & 13.48 & 13.57 & 13.21 & 0.4008 & 0.3991 & 41.94 & 29.00 \\
\hline $2 \mathrm{H}-1,47$ & 11.97 & 13.65 & 13.70 & 13.38 & 0.4049 & 0.4050 & 41.94 & 29.00 \\
\hline $2 \mathrm{H}-\mathrm{I}, 62$ & 12.12 & 13.80 & 13.78 & 13.53 & 0.4088 & 0.4114 & 38.81 & 23.28 \\
\hline $2 \mathrm{H}-1.77$ & 12.27 & 13.95 & 13.92 & 13.68 & 0.4145 & 0.4179 & 26.11 & 23.28 \\
\hline $2 \mathrm{H}-1.92$ & 12.42 & 14.10 & 14.11 & 13.83 & 0.4203 & 0.4243 & 26.11 & 23.28 \\
\hline $2 \mathrm{H}-1,107$ & 12.57 & 14.25 & 14.25 & 13.98 & 0.4260 & 0.4308 & 26.11 & 23.28 \\
\hline $2 \mathrm{H}-\mathrm{I} .122$ & 12.72 & 14.40 & 14.39 & 14.13 & 0.4317 & 0.4374 & 26.11 & 22.78 \\
\hline $2 \mathrm{H}-1,137$ & 12.87 & 14.55 & 14.56 & 14.28 & 0.4375 & 0.4441 & 26.11 & 22.30 \\
\hline $2 \mathrm{H}-2,2$ & 13.02 & 14.70 & 14.70 & 14.43 & 0.4432 & 0.4508 & 26.11 & 22.30 \\
\hline $2 \mathrm{H}-2.17$ & 13.17 & 14.85 & 14.85 & 14.58 & 0.4490 & 0.4575 & 26.11 & 22.30 \\
\hline $2 \mathrm{H}-2,30$ & 13.30 & 14.98 & 14.98 & 14.71 & 0.4540 & 0.4634 & 26.11 & 22.30 \\
\hline $2 \mathrm{H}-2,47$ & 13.47 & 15.15 & 15.15 & 14.88 & 0.4605 & 0.4710 & 26.11 & 22.30 \\
\hline $2 \mathrm{H}-2.64$ & 13.64 & 15.32 & 15.32 & 15.05 & 0.4653 & 0,4750 & 35.00 & 42.86 \\
\hline $2 \mathrm{H}-2.77$ & 13.77 & 15.45 & 15.45 & 15.18 & 0.4687 & 0.4780 & 39.09 & 42.86 \\
\hline $2 \mathrm{H}-2.92$ & 13.92 & 15.60 & 15.60 & 15.33 & 0.4725 & 0.4823 & 39.09 & 34.62 \\
\hline $2 \mathrm{H}-2.107$ & 14.07 & 15.75 & 15.75 & 15.48 & 0.4763 & 0.4867 & 39.09 & 34.62 \\
\hline $2 \mathrm{H}-2,122$ & 14.22 & 15.90 & 15.90 & 15.63 & 0.4802 & 0.4910 & 39.09 & 34.62 \\
\hline $2 \mathrm{H}-2.137$ & 14.37 & 16.05 & 16.05 & 15.78 & 0.4840 & 0.4984 & 39.09 & 20.15 \\
\hline $2 \mathrm{H}-3,2$ & 14.52 & 16.20 & 16.20 & 15.93 & 0.4887 & 0.5059 & 31.70 & 20.15 \\
\hline $2 \mathrm{H}-3,17$ & 14.67 & 16.35 & 16.35 & 16.08 & 0.4935 & 0.5133 & 31.70 & 20.15 \\
\hline $2 \mathrm{H}-3,30$ & 14.82 & 16.48 & 16.48 & 16.21 & 0.4976 & 0.5198 & 31.70 & 20.15 \\
\hline $2 \mathrm{H}-3.47$ & 14.97 & 16.65 & 16.65 & 16.38 & 0.5029 & 0.5267 & 31.70 & 24.53 \\
\hline $2 \mathrm{H}-3,62$ & 15.12 & 16.80 & 16.80 & 16.53 & 0.5077 & 0.5315 & 31.70 & 31.34 \\
\hline $2 \mathrm{H}-3,77$ & 15.27 & 16.95 & 16.96 & 16.68 & 0.5124 & 0.5363 & 31.70 & 31.34 \\
\hline $2 \mathrm{H}-3.92$ & 15.42 & 17.10 & 17.10 & 16.83 & 0.5171 & 0.5411 & 31.70 & 31.34 \\
\hline $2 \mathrm{H}-3,107$ & 15.57 & 17.25 & 17.24 & 16.98 & 0.5218 & 0.5459 & 31.70 & 31.34 \\
\hline $2 \mathrm{H}-3,122$ & 15.72 & 17.40 & 17.40 & 17.13 & 0.5266 & 0.5506 & 31.70 & 31.34 \\
\hline $2 \mathrm{H}-3,137$ & 15.87 & 17.55 & 17.57 & 17.28 & 0.5346 & 0.5554 & 18.81 & 31.34 \\
\hline $2 \mathrm{H}-4.2$ & 16.02 & 17.70 & 17.71 & 17.43 & 0.5439 & 0.5602 & 16.02 & 31.34 \\
\hline $2 \mathrm{H}-4,17$ & 16.17 & 17.85 & 17.85 & 17.58 & 0.5533 & 0.5650 & 16.02 & 31.34 \\
\hline $2 \mathrm{H}-4,32$ & 16.32 & 18.00 & 17.99 & 17.73 & 0.5626 & 0.5686 & 16.02 & 41.67 \\
\hline $2 \mathrm{H}-4.47$ & 16.47 & 18.15 & 18.14 & 17.88 & 0.5720 & 0.5722 & 16.02 & 41.67 \\
\hline $2 \mathrm{H}-4,62$ & 16.62 & 18.30 & 18.31 & 18.03 & 0.5794 & 0.5768 & 20.38 & 32.97 \\
\hline $2 \mathrm{H}-4.77$ & 16.77 & 18.45 & 18.46 & 18.18 & 0.5844 & 0.5823 & 29.58 & 27.27 \\
\hline $2 \mathrm{H}-4,92$ & 16.92 & 18.60 & 18.60 & 18.33 & 0.5895 & 0.5878 & 29.58 & 27.27 \\
\hline $2 \mathrm{H}-4.107$ & 17.07 & 18.75 & 18.76 & 18.48 & 0.5946 & 0.5933 & 29.58 & 27.27 \\
\hline $2 \mathrm{H}-4,122$ & 17.22 & 18.90 & 18.91 & 18.63 & 0.5997 & 0.5988 & 29.58 & 27.27 \\
\hline $2 \mathrm{H}-4,137$ & 17.37 & 19.05 & 19.05 & 18.78 & 0.6047 & 0.6043 & 29.58 & 27.27 \\
\hline $2 \mathrm{H}-5,2$ & 17.52 & 19.20 & 19.20 & 18.93 & 0.6098 & 0.6103 & 29.58 & 24.66 \\
\hline $2 \mathrm{H}-5,17$ & 17.67 & 19.35 & 19.36 & 19.08 & 0.6149 & 0.6170 & 29.58 & 22.50 \\
\hline $2 \mathrm{H}-5,30$ & 17.82 & 19.48 & 19.49 & 19.21 & 0.6193 & 0.6210 & 29.58 & 32.14 \\
\hline $2 \mathrm{H}-5,47$ & 17.97 & 19.65 & 19.65 & 19.38 & 0.6250 & 0.6263 & 29.58 & 32.14 \\
\hline $2 \mathrm{H}-5,62$ & 18.12 & 19.80 & 19.79 & 19.53 & 0.6301 & 0.6310 & 29.58 & 32.14 \\
\hline $2 \mathrm{H}-5,77$ & 18.27 & 19.95 & 19.95 & 19.68 & 0.6352 & 0.6366 & 29.58 & 26.61 \\
\hline $2 \mathrm{H}-5,92$ & 18.42 & 20.10 & 20.10 & 19.83 & 0.6402 & 0.6423 & 29.58 & 26.61 \\
\hline $2 \mathrm{H}-5,107$ & 18.57 & 20.25 & 20.25 & 19.98 & 0.6453 & 0.6479 & 29.58 & 26.61 \\
\hline $2 \mathrm{H}-5,122$ & 18.72 & 20.40 & 20.40 & 20.13 & 0.6509 & 0.6535 & 26.87 & 26.61 \\
\hline $2 \mathrm{H}-5,137$ & 18.87 & 20.55 & 20.55 & 20.28 & 0.6570 & 0.6592 & 24.32 & 26.61 \\
\hline $2 \mathrm{H}-6.2$ & 19.02 & 20.70 & 20.70 & 20.43 & 0.6632 & 0.6648 & 24.32 & 26.61 \\
\hline $2 \mathrm{H}-6,17$ & 19.17 & 20.85 & 20.87 & 20.58 & 0.6694 & 0.6705 & 24.32 & 26.61 \\
\hline $2 \mathrm{H}-6,30$ & 19.32 & 20.98 & 21.00 & 20.71 & 0.6747 & 0.6753 & 24.32 & 26.61 \\
\hline $2 \mathrm{H}-6.47$ & 19.47 & 21.15 & 21.14 & 20.88 & 0.6817 & 0.6817 & 24.32 & 26.61 \\
\hline $2 \mathrm{H}-6,64$ & 19.62 & 21.32 & 21.35 & 21.05 & 0.6887 & 0.6881 & 24.32 & 26.61 \\
\hline $2 \mathrm{H}-6.77$ & 19.77 & 21.45 & 21.55 & 21.18 & 0.6970 & 0.6930 & 15.68 & 26.61 \\
\hline $2 \mathrm{H}-6,92$ & 19.92 & 21.60 & 21.71 & 21.33 & 0.7120 & 0.6984 & 10.00 & 27.78 \\
\hline $2 \mathrm{H}-6,107$ & 20.07 & 21.75 & 21.84 & 21.48 & 0.7174 & 0.7038 & 27.57 & 27.78 \\
\hline $2 \mathrm{H}-6,122$ & 20.22 & 21.90 & 22.02 & 21.63 & 0.7229 & 0.7092 & 27.57 & 27.78 \\
\hline $2 \mathrm{H}-6.137$ & 20.37 & 22.05 & 22.19 & 21.78 & 0.7283 & 0.7146 & 27.57 & 27.78 \\
\hline $2 \mathrm{H}-7,2$ & 20.52 & 22.20 & 22.34 & 21.93 & 0.7338 & 0.7200 & 27.57 & 27.78 \\
\hline $2 \mathrm{H}-7,17$ & 20.67 & 22.35 & 22.46 & 22.08 & 0.7392 & 0.7264 & 27.57 & 23.33 \\
\hline $2 \mathrm{H}-7,30$ & 20.82 & 22.48 & 22.54 & 22.21 & 0.7439 & 0.7320 & 27.57 & 23.33 \\
\hline 138-847D- & & & & & & & & \\
\hline $3 \mathrm{H}-3,62$ & 19.72 & 22.72 & 22.72 & 22.38 & 0.7497 & 0.7385 & 29.62 & 26.11 \\
\hline $3 \mathrm{H}-3,77$ & 19.87 & 22.87 & 22.86 & 22.53 & 0.7530 & 0.7443 & 45.39 & 26.11 \\
\hline $3 \mathrm{H}-3,92$ & 20.02 & 23.02 & 23.01 & 22.68 & 0.7563 & 0.7500 & 45.39 & 26.11 \\
\hline $3 \mathrm{H}-3,107$ & 20.17 & 23.17 & 23.17 & 22.83 & 0.7596 & 0.7553 & 45.39 & 28.18 \\
\hline $3 \mathrm{H}-3,122$ & 20.32 & 23.32 & 23.32 & 22.98 & 0.7629 & 0.7606 & 45.39 & 28.18 \\
\hline $3 \mathrm{H}-3,137$ & 20.47 & 23.47 & 23.47 & 23.13 & 0.7662 & 0.7660 & 45.39 & 28.18 \\
\hline
\end{tabular}


Table 1 (continued).

\begin{tabular}{|c|c|c|c|c|c|c|c|c|}
\hline \multirow[b]{2}{*}{$\begin{array}{l}\text { Core, section, } \\
\text { interval }(\mathrm{cm})\end{array}$} & \multirow[b]{2}{*}{$\begin{array}{l}\text { ODP } \\
\text { depth } \\
\text { (mbsf) }\end{array}$} & \multirow{2}{*}{$\begin{array}{l}\text { Shipboard } \\
\text { composite } \\
\text { depth } \\
\text { (mcd) }\end{array}$} & & e & $\mathrm{Ag}$ & & Sediment: & ion rates \\
\hline & & & $\begin{array}{l}\text { composite } \\
\text { depth } \\
\text { (rmcd) }\end{array}$ & $\begin{array}{l}\text { depth. } \\
\text { this study } \\
\text { (Bmed) }\end{array}$ & $\begin{array}{l}\text { Shackleton } \\
\text { age model } \\
\text { (Ma) }\end{array}$ & $\begin{array}{c}\text { Isotope } \\
\text { age model } \\
(\mathrm{Ma})\end{array}$ & $\begin{array}{c}\text { Shackleton } \\
\text { ages } \\
(\mathrm{m} / \mathrm{m} . \mathrm{y} \text {.) }\end{array}$ & $\begin{array}{c}\text { Isotope } \\
\text { ages } \\
\text { (m/m.y.) }\end{array}$ \\
\hline $138-847 \mathrm{C}-$ & & & & & & & & \\
\hline $3 \mathrm{H}-\mathrm{I}, 17$ & 21.17 & 23.57 & 23.48 & 23.31 & $0.770 \mathrm{I}$ & 0.7724 & 45.39 & 28.18 \\
\hline $3 \mathrm{H}-\mathrm{I}, 32$ & 21.32 & 23.72 & 23.62 & 23.46 & 0.7735 & 0.7777 & 45.39 & 28.18 \\
\hline $3 \mathrm{H}-1.47$ & 21.47 & 23.87 & 23.72 & 23.61 & 0.7768 & 0.7830 & 45.39 & 28.18 \\
\hline $3 \mathrm{H}-1.62$ & 21.62 & 24.02 & 23.84 & 23.76 & 0.7801 & 0.7880 & 45.39 & 30.00 \\
\hline $3 \mathrm{H}-1.77$ & 21.77 & 24.17 & 24.01 & 23.91 & 0.7834 & 0.7930 & 45.39 & 30.00 \\
\hline $3 \mathrm{H}-\mathrm{I} .92$ & 21.92 & 24.32 & 24.27 & 24.06 & 0.7867 & 0.7980 & 45.39 & 30.00 \\
\hline $3 \mathrm{H}-1.107$ & 22.07 & 24.47 & 24.47 & 24.21 & 0.7931 & 0.8028 & 23.22 & 31.54 \\
\hline $3 \mathrm{H}-\mathrm{I}, 122$ & 22.22 & 24.62 & 24.62 & 24.36 & 0.7999 & 0.8075 & 22.03 & 31.54 \\
\hline $3 \mathrm{H}-1.137$ & 22.37 & 24.77 & 24.77 & 24.51 & 0.8067 & 0.8123 & 22.03 & 31.54 \\
\hline $3 \mathrm{H}-2,2$ & 22.52 & 24,92 & 24.92 & 24.66 & 0.8136 & 0.8170 & 22.03 & 31.54 \\
\hline $3 \mathrm{H}-2.17$ & 22.67 & 25.07 & 25.07 & 24.81 & 0.8204 & 0.8218 & 22.03 & 31.54 \\
\hline $3 \mathrm{H}-2.30$ & 22.82 & 25.20 & 25.20 & 24.94 & 0.8254 & 0.8259 & 25.84 & 31.54 \\
\hline $3 \mathrm{H}-2,47$ & 22.97 & 25.37 & 25.37 & 25.11 & 0.8302 & 0.8313 & 35.71 & 31.54 \\
\hline $3 \mathrm{H}-2.65$ & 23.12 & 25.55 & 25.55 & 25.29 & 0.8352 & 0.8370 & 35.71 & 31.54 \\
\hline $3 \mathrm{H}-2,77$ & 23.27 & 25.67 & 25.67 & 25.41 & 0.8386 & 0.8411 & 35.71 & 29.33 \\
\hline $3 \mathrm{H}-2,92$ & 23.42 & 25.82 & 25.82 & 25.56 & 0.8428 & 0.8462 & 35.71 & 29.33 \\
\hline $3 \mathrm{H}-2,107$ & 23.57 & 25.97 & 25.97 & 25.71 & 0.8470 & 0.8513 & 35.71 & 29.33 \\
\hline $3 \mathrm{H}-2,122$ & 23.72 & 26.12 & 26.12 & 25.86 & 0.8512 & 0.8564 & 35.71 & 29.33 \\
\hline $3 \mathrm{H}-2,137$ & 23.87 & 26.27 & 26.27 & 26.01 & 0.8554 & 0.8615 & 35.71 & 29.33 \\
\hline $3 \mathrm{H}-3.2$ & 24.02 & 26.42 & 26.42 & 26.16 & 0.8596 & 0.8667 & 35.71 & 29.33 \\
\hline $3 \mathrm{H}-3.17$ & 24.17 & 26.57 & 26.57 & 26.31 & 0.8638 & 0.8718 & 35.71 & 29.33 \\
\hline $3 \mathrm{H}-3,32$ & 24.32 & 26.72 & 26.72 & 26.46 & 0.8680 & 0.8769 & 35.71 & 29.33 \\
\hline $3 \mathrm{H}-3,47$ & 24.47 & 26.87 & 26.87 & 26.61 & 0.8722 & 0.8820 & 35.71 & 29.33 \\
\hline $3 \mathrm{H}-3,62$ & 24.62 & 27.02 & 27.02 & 26.76 & 0.8764 & 0.8868 & 35.71 & 31.25 \\
\hline $3 \mathrm{H}-3.76$ & 24.76 & 27.16 & 27.16 & 26.90 & 0.8803 & 0.8913 & 35.71 & 31.25 \\
\hline $3 \mathrm{H}-3,92$ & 24.92 & 27.32 & 27.32 & 27.06 & 0.8848 & 0.8964 & 35.71 & 31.25 \\
\hline $3 \mathrm{H}-3, \mathrm{I} 07$ & 25.07 & 27.47 & 27.47 & 27.21 & 0.8890 & 0.9012 & 35.71 & 31.25 \\
\hline $3 \mathrm{H}-3,122$ & 25.22 & 27.62 & 27.61 & 27.36 & 0.8932 & 0.9060 & 35.71 & 31.25 \\
\hline $3 \mathrm{H}-3,137$ & 25.37 & 27.77 & 27.75 & 27.51 & 0.8974 & 0.9100 & 35.71 & 37.50 \\
\hline $3 \mathrm{H}-4,2$ & 25.52 & 27.92 & 27.91 & 27.66 & 0.9016 & 0.9140 & 35.71 & 37.50 \\
\hline $3 \mathrm{H}-4,17$ & 25.67 & 28.07 & 28.06 & 27.81 & 0.9058 & 0.9180 & 35.71 & 37.50 \\
\hline $3 \mathrm{H}-4,32$ & 25.82 & 28.22 & 28.22 & 27.96 & 0.9114 & 0.9233 & 26.80 & 28.13 \\
\hline $3 \mathrm{H}-4,47$ & 25.97 & 28.37 & 28.37 & 28.11 & 0.9185 & 0.9287 & 20.86 & 28.13 \\
\hline $3 \mathrm{H}-4,62$ & 26.12 & 28.52 & 28.52 & 28.26 & 0.9257 & 0.9340 & 20.86 & 28.13 \\
\hline $3 \mathrm{H}-4,76$ & 26.26 & 28.66 & 28.66 & 28.40 & 0.9325 & 0.9390 & 20.86 & 28.13 \\
\hline $3 \mathrm{H}-4.92$ & 26.42 & 28.82 & 28.82 & 28.56 & 0.9401 & 0.9447 & 20.86 & 28.13 \\
\hline $3 \mathrm{H}-4,107$ & 26.57 & 28.97 & 28.97 & 28.71 & 0.9473 & 0.9500 & 20.86 & 28.13 \\
\hline $3 \mathrm{H}-4,122$ & 26.72 & 29.12 & 29.12 & 28.86 & 0.9545 & 0.9543 & 20.86 & 35.00 \\
\hline $3 \mathrm{H}-4,137$ & 26.87 & 29.27 & 29.27 & 29.01 & 0.9617 & 0.9586 & 20.86 & 35.00 \\
\hline $3 \mathrm{H}-5,2$ & 27.02 & 29.42 & 29.42 & 29.16 & 0.9689 & 0.9629 & 20.86 & 35.00 \\
\hline $3 \mathrm{H}-5,17$ & 27.17 & 29.57 & 29.57 & 29.31 & 0.9761 & 0.9694 & 20.86 & 22.93 \\
\hline $3 \mathrm{H}-5,32$ & 27.32 & 29.72 & 29.72 & 29.46 & 0.9810 & 0.9782 & 30.81 & 17.05 \\
\hline $3 \mathrm{H}-5,47$ & 27.47 & 29.87 & 29.87 & 29.61 & 0.9850 & 0.9870 & 37.27 & 17.05 \\
\hline $3 \mathrm{H}-5,62$ & 27.62 & 30.02 & 30.02 & 29.76 & 0.9890 & 0.9900 & 37.27 & 50.00 \\
\hline $3 \mathrm{H}-5,76$ & 27.76 & 30.16 & 30.16 & 29.90 & 0.9928 & 0.9928 & 37.27 & 50.00 \\
\hline $3 \mathrm{H}-5.92$ & 27.92 & 30.32 & 30.32 & 30.06 & 0.9970 & 0.9960 & 37.27 & 50.00 \\
\hline $3 \mathrm{H}-5,107$ & 28.07 & 30.47 & 30.47 & 30.21 & 1.0018 & 0.9990 & 31.45 & 50.00 \\
\hline $3 \mathrm{H}-5,122$ & 28.22 & 30.62 & 30.62 & 30.36 & 1.0086 & 1.0020 & 22.00 & 50.00 \\
\hline $3 \mathrm{H}-5,137$ & 28.37 & 30.77 & 30.77 & 30.51 & 1.0155 & 1.0050 & 22.00 & 50.00 \\
\hline $3 \mathrm{H}-6,2$ & 28.52 & 30.92 & 30.92 & 30.66 & 1.0223 & 1.0100 & 22.00 & 30.29 \\
\hline $3 \mathrm{H}-6.17$ & 28.67 & 31.07 & 31.07 & 30.81 & 1.0291 & 1.0149 & 22.00 & 30.29 \\
\hline $3 \mathrm{H}-6,30$ & 28.82 & 31.20 & 31.20 & 30.94 & 1.0350 & 1.0192 & 22.00 & 30.29 \\
\hline $3 \mathrm{H}-6,47$ & 28.97 & 31.37 & 31.37 & 31.11 & 1.0427 & 1.0255 & 22.00 & 26.98 \\
\hline $3 \mathrm{H}-6.65$ & 29.12 & 31.55 & 31.55 & 31.29 & 1.0504 & 1.0329 & 23.43 & 24.32 \\
\hline $3 \mathrm{H}-6,77$ & 29.27 & 31.67 & 31.66 & 31.41 & 1.0529 & 1.0378 & 48.64 & 24.32 \\
\hline $3 \mathrm{H}-6,92$ & 29.42 & 31.82 & 31.81 & 31.56 & 1.0560 & 1.0440 & 48.64 & 24.32 \\
\hline $3 \mathrm{H}-6.107$ & 29.57 & 31.97 & 31.99 & 31.71 & 1.0590 & 1.0474 & 48.64 & 43.75 \\
\hline $3 \mathrm{H}-6,122$ & 29.72 & 32.12 & 32.17 & 31.86 & 1.0621 & 1.0509 & 48.64 & 43.75 \\
\hline $3 \mathrm{H}-6,137$ & 29.87 & 32.27 & 32.34 & 32.01 & 1.0652 & 1.0543 & 48.64 & 43.75 \\
\hline $3 \mathrm{H}-7.2$ & 30.02 & 32.42 & 32.48 & 32.16 & 1.0683 & 1.0577 & 48.64 & 43.75 \\
\hline $3 \mathrm{H}-7,17$ & 30.17 & 32.57 & 32.59 & 32.31 & 1.0714 & 1.0611 & 48.64 & 43.75 \\
\hline $3 \mathrm{H}-7.30$ & 30.30 & 32.70 & 32.68 & 32.44 & 1.0747 & 1.0641 & 39.19 & 43.75 \\
\hline $3 \mathrm{H}-7.47$ & 30.47 & 32.87 & 32.84 & 32.61 & 1.0793 & 1.0680 & 37.03 & 43.75 \\
\hline $3 \mathrm{H}-7.62$ & 30.62 & 33.02 & 33.05 & 32.76 & 1.0833 & 1.0728 & 37.03 & 31.48 \\
\hline 138-847D- & & & & & & & & \\
\hline $4 \mathrm{H}-3,47$ & 29.07 & 33.07 & 33.07 & 32.86 & 1.0860 & 1.0759 & 37.03 & 31.48 \\
\hline $4 \mathrm{H}-3,62$ & 29.22 & 33.22 & 33.22 & 33.01 & 1.0901 & 1.0807 & 37.03 & 31.48 \\
\hline $4 \mathrm{H}-3,77$ & 29.37 & 33.37 & 33.37 & 33.16 & 1.0941 & 1.0855 & 37.03 & 31.48 \\
\hline $4 \mathrm{H}-3.92$ & 29.52 & 33.52 & 33.51 & 33.31 & 1.0982 & 1.0902 & 37.03 & 31.48 \\
\hline $4 \mathrm{H}-3,107$ & 29.67 & 33.67 & 33.66 & 33.46 & 1.1022 & 1.0950 & 37.03 & 31.48 \\
\hline $4 \mathrm{H}-3,122$ & 29.82 & 33.82 & 33.82 & 33.61 & 1.1063 & 1.0993 & 37.03 & 35.00 \\
\hline $4 \mathrm{H}-3,137$ & 29.97 & 33.97 & 33.97 & 33.76 & 1.1103 & 1.1036 & 37.03 & 35.00 \\
\hline $138-847 \mathrm{C}$ - & & & & & & & & \\
\hline $4 \mathrm{H}-1,32$ & 30.82 & 34.12 & 34.22 & 33.94 & 1.1152 & 1.1087 & 37.03 & 35.00 \\
\hline $4 \mathrm{H}-1.47$ & 30.97 & 34.27 & 34.58 & 34.09 & 1.1193 & 1.1130 & 37.03 & 35.00 \\
\hline $4 \mathrm{H}-1,61$ & 31.11 & 34.41 & 34.68 & 34.23 & 1.1230 & 1.1174 & 37.03 & 32.14 \\
\hline $4 \mathrm{H}-\mathrm{I}, 77$ & 31.27 & 34.57 & 34.76 & 34.39 & 1.1274 & 1.1223 & 37.03 & 32.14 \\
\hline $4 \mathrm{H}-1,92$ & 31.42 & 34.72 & 34.85 & 34.54 & 1.1314 & 1.1270 & 37.03 & 32.14 \\
\hline $4 \mathrm{H}-\mathrm{I}, 107$ & 31.57 & 34.87 & 34.95 & 34.69 & 1.1355 & 1.1323 & 37.03 & 28.13 \\
\hline $4 \mathrm{H}-1,122$ & 31.72 & 35.02 & 35.08 & 34.84 & 1.1395 & 1.1377 & 37.03 & 28.12 \\
\hline $4 \mathrm{H}-1,137$ & 31.87 & 35.17 & 35.22 & 34.99 & 1.1436 & 1.1430 & 37.03 & 28.13 \\
\hline
\end{tabular}


Table 1 (continued).

\begin{tabular}{|c|c|c|c|c|c|c|c|c|}
\hline \multirow[b]{2}{*}{$\begin{array}{l}\text { Core, section, } \\
\text { interval }(\mathrm{cm})\end{array}$} & \multirow[b]{2}{*}{$\begin{array}{c}\text { ODP } \\
\text { depth } \\
\text { (mbsf) }\end{array}$} & \multirow{2}{*}{$\begin{array}{c}\text { Shipboard } \\
\text { composite } \\
\text { depth } \\
\text { (mcd) }\end{array}$} & Revised & Composite & $\mathrm{Ag}$ & & Sedimenta & ion rates \\
\hline & & & $\begin{array}{l}\text { composite } \\
\text { depth } \\
\text { (rmcd) }\end{array}$ & $\begin{array}{l}\text { depth, } \\
\text { this study } \\
\text { (Bmcd) }\end{array}$ & $\begin{array}{l}\text { Shackleton } \\
\text { age model } \\
\text { (Ma) }\end{array}$ & $\begin{array}{c}\text { Isotope } \\
\text { age model } \\
\text { (Ma) }\end{array}$ & $\begin{array}{c}\text { Shackleton } \\
\text { ages } \\
(\mathrm{m} / \mathrm{m} . \mathrm{y} .)\end{array}$ & $\begin{array}{l}\text { Isotope } \\
\text { ages } \\
\text { (m/m.y.) }\end{array}$ \\
\hline $4 \mathrm{H}-2,2$ & 32.02 & 35.32 & 35.34 & 35.14 & 1.1476 & - & 37.03 & - \\
\hline $4 \mathrm{H}-2,17$ & 32.17 & 35.47 & 35.48 & 35.29 & 1.1517 & - & 37.03 & - \\
\hline $4 \mathrm{H}-2,32$ & 32.32 & 35.62 & 35.63 & 35.44 & 1.1557 & - & 37.03 & - \\
\hline $4 \mathrm{H}-2,47$ & 32.47 & 35.77 & 35.78 & 35.59 & 1.1598 & - & 37.03 & - \\
\hline $4 \mathrm{H}-2,63.5$ & 32.635 & 35.94 & 35.93 & 35.76 & 1.1642 & - & 37.03 & - \\
\hline $4 \mathrm{H}-2.77$ & 32.77 & 36.07 & 36.02 & 35.89 & 1.1679 & - & 37.03 & - \\
\hline $4 \mathrm{H}-2,88$ & 32.88 & 36.18 & 36.10 & 36.00 & 1.1708 & - & 37.03 & - \\
\hline $4 \mathrm{H}-2,107$ & 33.07 & 36.37 & 36.26 & 36.19 & 1.1760 & - & 37.03 & - \\
\hline $4 \mathrm{H}-2,122$ & 33.22 & 36.52 & 36.39 & 36.34 & 1.1800 & - & 37.03 & - \\
\hline $4 \mathrm{H}-2,137$ & 33.37 & 36.67 & 36.50 & 36.49 & 1.1841 & - & 37.03 & - \\
\hline $4 \mathrm{H}-3,2$ & 33.52 & 36.82 & 36.59 & 36.64 & 1.1881 & - & 37.03 & - \\
\hline $4 \mathrm{H}-3,17$ & 33.67 & 36.97 & 36.68 & 36.79 & 1.1922 & - & 37.03 & - \\
\hline $4 \mathrm{H}-3,32$ & 33.82 & 37.12 & 36.78 & 36.94 & 1.1962 & - & 37.03 & - \\
\hline $4 \mathrm{H}-3,47$ & 33.97 & 37.27 & 36.99 & 37.09 & 1.2003 & - & 37.03 & - \\
\hline $4 \mathrm{H}-3,61$ & 34.11 & 37.41 & 37.42 & 37.23 & 1.2041 & - & 37.03 & - \\
\hline $4 \mathrm{H}-3,77$ & 34.27 & 37.57 & 37.84 & 37.39 & 1.2074 & - & 48.10 & - \\
\hline $4 \mathrm{H}-3,92$ & 34.42 & 37.72 & 37.97 & 37.54 & 1.2102 & - & 52.50 & 一 \\
\hline $4 \mathrm{H}-3,107$ & 34.57 & 37.87 & 38.07 & 37.69 & 1.2131 & - & 52.50 & - \\
\hline $4 \mathrm{H}-3,122$ & 34.72 & 38.02 & 38.19 & 37.84 & 1.2162 & - & 48.04 & - \\
\hline $4 \mathrm{H}-3,137$ & 34.87 & 38.17 & 38.33 & 37.99 & 1.2199 & - & 41.07 & - \\
\hline $4 \mathrm{H}-4.2$ & 35.02 & 38.32 & 38.48 & 38.14 & 1.2235 & - & 41.07 & - \\
\hline $4 \mathrm{H}-4,17$ & 35.17 & 38.47 & 38.61 & 38.29 & 1.2272 & - & 41.07 & - \\
\hline $4 \mathrm{H}-4,30$ & 35.30 & 38.60 & 38.72 & 38.42 & 1.2303 & - & 41.07 & - \\
\hline $4 \mathrm{H}-4,47$ & 35.47 & 38.77 & 38.86 & 38.59 & 1.2345 & - & 41.07 & - \\
\hline $4 \mathrm{H}-4.61$ & 35.61 & 38.91 & 38.96 & 38.73 & 1.2379 & - & 41.07 & - \\
\hline $4 \mathrm{H}-4,77$ & 35.77 & 39.07 & 39.06 & 38.89 & 1.2418 & - & 41.07 & - \\
\hline $4 \mathrm{H}-4,92$ & 35.92 & 39.22 & 39.15 & 39.04 & 1.2470 & - & 28.90 & - \\
\hline $4 \mathrm{H}-4,107$ & 36.07 & 39.37 & 39.25 & 39.19 & 1.2529 & - & 25.17 & - \\
\hline $4 \mathrm{H}-4,122$ & 36.22 & 39.52 & 39.37 & 39.34 & 1.2589 & - & 25.17 & - \\
\hline $4 \mathrm{H}-4,137$ & 36.37 & 39.67 & 39.52 & 39.49 & 1.2648 & - & 25.17 & - \\
\hline $4 \mathrm{H}-5,2$ & 36.52 & 39.82 & 39.73 & 39.64 & 1.2708 & - & 25.17 & - \\
\hline $4 \mathrm{H}-5,17$ & 36.67 & 39.97 & 39.95 & 39.79 & 1.2742 & - & 44.22 & - \\
\hline $4 \mathrm{H}-5,30$ & 36.80 & 40.10 & 40.17 & 39.92 & 1.2766 & - & 54.55 & - \\
\hline $4 \mathrm{H}-5,47$ & 36.97 & 40.27 & 40.38 & 40.09 & 1.2797 & - & 54.55 & - \\
\hline $4 \mathrm{H}-5,61$ & 37.11 & 40.41 & 40.48 & 40.23 & 1.2823 & - & 54.55 & - \\
\hline $4 \mathrm{H}-5.77$ & 37.27 & 40.57 & 40.59 & 40.39 & 1.2875 & - & 30.78 & - \\
\hline $4 \mathrm{H}-5,92$ & 37.42 & 40.72 & 40.68 & 40.54 & 1.2930 & - & 26.87 & - \\
\hline $4 \mathrm{H}-5,107$ & 37.57 & 40.87 & 40.79 & 40.69 & 1.2986 & - & 26.88 & - \\
\hline $4 \mathrm{H}-5,122$ & 37.72 & 41.02 & 40.95 & 40.84 & 1.3042 & - & 26.87 & - \\
\hline $4 \mathrm{H}-5,137$ & 37.87 & 41.17 & 41.19 & 40.99 & 1.3097 & - & 27.55 & - \\
\hline $4 \mathrm{H}-6,2$ & 38.02 & 41.32 & 41.41 & 41.14 & 1.3150 & - & 28.25 & - \\
\hline $4 \mathrm{H}-6,17$ & 38.17 & 41.47 & 41.56 & 41.29 & 1.3203 & - & 28.25 & - \\
\hline $4 \mathrm{H}-6,29$ & 38.29 & 41.59 & 41.66 & 41.41 & 1.3245 & - & 28.25 & - \\
\hline $4 \mathrm{H}-6,47$ & 38.47 & 41.77 & 41.85 & 41.59 & 1.3285 & - & 45.63 & - \\
\hline $4 \mathrm{H}-6,64$ & 38.64 & 41.94 & 42.05 & 41.76 & 1.3307 & - & 75.00 & - \\
\hline $4 \mathrm{H}-6,77$ & 38.77 & 42.07 & 42.19 & 41.89 & 1.3325 & - & 75.00 & - \\
\hline $4 \mathrm{H}-6,92$ & 38.92 & 42.22 & 42.30 & 42.04 & 1.3345 & - & 75.00 & - \\
\hline 138-847D- & & & & & & & & \\
\hline $4 \mathrm{H}-6,107$ & 39.07 & 42.37 & 42.39 & 42.19 & 1.3365 & - & 75.00 & - \\
\hline $4 \mathrm{H}-6,122$ & 39.22 & 42.52 & 42.46 & 42.34 & 1.3403 & - & 38.65 & - \\
\hline $4 \mathrm{H}-6,137$ & 39.37 & 42.67 & 42.55 & 42.49 & 1.3449 & - & 32.86 & - \\
\hline $4 \mathrm{H}-7,2$ & 39.52 & 42.82 & 42.69 & 42.64 & 1.3495 & - & 32.86 & - \\
\hline $4 \mathrm{H}-7,17$ & 39.67 & 42.97 & 42.99 & 42.79 & 1.3540 & - & 32.86 & - \\
\hline $4 \mathrm{H}-7,32$ & 39.82 & 43.12 & 43.36 & 42.94 & 1.3586 & - & 32.86 & - \\
\hline $4 \mathrm{H}-7,47$ & 39.97 & 43.27 & 43.46 & 43.09 & 1.3632 & - & 32.86 & - \\
\hline $5 \mathrm{H}-3,107$ & 39.17 & 43.37 & 43.38 & 43.17 & 1.3656 & - & 32.86 & - \\
\hline $5 \mathrm{H}-3,122$ & 39.32 & 43.52 & 43.53 & 43.32 & 1.3702 & - & 32.86 & - \\
\hline $5 \mathrm{H}-3,137$ & 39.47 & 43.67 & 43.67 & 43.47 & 1.3747 & - & 32.86 & - \\
\hline $5 \mathrm{H}-4,2$ & 39.62 & 43.82 & 43.82 & 43.62 & 1.3792 & - & 33.44 & - \\
\hline $5 \mathrm{H}-4,17$ & 39.77 & 43.97 & 43.99 & 43.77 & 1.3826 & - & 44.52 & - \\
\hline $5 \mathrm{H}-4,32$ & 39.92 & 44.12 & 44.14 & 43.92 & 1.3860 & - & 44.52 & - \\
\hline $5 \mathrm{H}-4,47$ & 40.07 & 44.27 & 44.27 & 44.07 & 1.3893 & - & 44.52 & - \\
\hline $5 \mathrm{H}-4.62$ & 40.22 & 44.42 & 44.41 & 44.22 & 1.3927 & - & 44.52 & - \\
\hline $5 \mathrm{H}-4,77$ & 40.37 & 44.57 & 44.55 & 44.37 & 1.3961 & - & 44.52 & - \\
\hline $5 \mathrm{H}-4,92$ & 40.52 & 44.72 & 44.72 & 44.52 & 1.3994 & - & 44.52 & - \\
\hline 138-847C & & & & & & & & \\
\hline $5 \mathrm{H}-1,47$ & 40.47 & 44.97 & 44.84 & 44.62 & 1.4030 & - & 28.23 & - \\
\hline $5 \mathrm{H}-1,62$ & 40.62 & 45.12 & 45.01 & 44.77 & 1.4089 & - & 25.16 & - \\
\hline $5 \mathrm{H}-1,77$ & 40.77 & 45.27 & 45.15 & 44.92 & 1.4149 & - & 25.16 & - \\
\hline $5 \mathrm{H}-1,92$ & 40.92 & 45.42 & 45.30 & 45.07 & 1.4209 & - & 25.16 & - \\
\hline $5 \mathrm{H}-1,107$ & 41.07 & 45.57 & 45.48 & 45.22 & 1.4268 & - & 25.16 & - \\
\hline $5 \mathrm{H}-1,122$ & 41.22 & 45.72 & 45.61 & 45.37 & 1.4325 & - & 26.46 & - \\
\hline $5 \mathrm{H}-1,137$ & 41.37 & 45.87 & 45.75 & 45.52 & 1.4375 & - & 30.08 & - \\
\hline $5 \mathrm{H}-2,2$ & 41.52 & 46.02 & 45.97 & 45.67 & 1.4425 & - & 30.08 & - \\
\hline $5 \mathrm{H}-2,17$ & 41.67 & 46.17 & 46.15 & 45.82 & 1.4475 & - & 30.08 & - \\
\hline $5 \mathrm{H}-2,28$ & 41.78 & 46.28 & 46.29 & 45.93 & 1.4511 & - & 30.08 & - \\
\hline $5 \mathrm{H}-2,47$ & 41.97 & 46.47 & 46.51 & 46.12 & 1.4574 & - & 30.08 & - \\
\hline $5 \mathrm{H}-2,64$ & 42.14 & 46.64 & 46.67 & 46.29 & 1.4631 & - & 30.08 & - \\
\hline $5 \mathrm{H}-2,77$ & 42.27 & 46.77 & 46.76 & 46.42 & 1.4674 & - & 30.08 & - \\
\hline $5 \mathrm{H}-2,92$ & 42.42 & 46.92 & 46.90 & 46.57 & 1.4724 & - & 30.08 & - \\
\hline $5 \mathrm{H}-2,107$ & 42.57 & 47.07 & 47.08 & 46.72 & 1.4774 & - & 30.08 & - \\
\hline $5 \mathrm{H}-2,122$ & 42.72 & 47.22 & 47.22 & 46.87 & 1.4824 & - & 30.08 & - \\
\hline
\end{tabular}


Table 1 (continued).

\begin{tabular}{|c|c|c|c|c|c|c|c|c|}
\hline \multirow[b]{2}{*}{$\begin{array}{l}\text { Core, section, } \\
\text { interval }(\mathrm{cm})\end{array}$} & \multirow[b]{2}{*}{$\begin{array}{c}\text { ODP } \\
\text { depth } \\
\text { (mbsf) }\end{array}$} & \multirow{2}{*}{$\begin{array}{l}\text { Shipboard } \\
\text { composite } \\
\text { depth } \\
\text { (mcd) }\end{array}$} & Revised & Composite & $\mathrm{Ag}$ & & Sediment: & ion rates \\
\hline & & & $\begin{array}{l}\text { composite } \\
\text { depth } \\
\text { (rmcd) }\end{array}$ & $\begin{array}{l}\text { depth, } \\
\text { this study } \\
\text { (Bmcd) }\end{array}$ & $\begin{array}{l}\text { Shackleton } \\
\text { age model } \\
\text { (Ma) }\end{array}$ & $\begin{array}{c}\text { Isotope } \\
\text { age model } \\
\text { (Ma) }\end{array}$ & $\begin{array}{l}\text { Shackleton } \\
\text { ages } \\
(\mathrm{m} / \mathrm{m} . \mathrm{y} .)\end{array}$ & $\begin{array}{c}\text { Isotope } \\
\text { ages } \\
\left(\mathrm{m} / \mathrm{m} . y_{*}\right)\end{array}$ \\
\hline $5 \mathrm{H}-2.137$ & 42.87 & 47.37 & 47.36 & 47.02 & 1.4873 & - & 30.08 & - \\
\hline $5 \mathrm{H}-3,2$ & 43.02 & 47.52 & 47.53 & 47.17 & 1.4923 & - & 30.08 & - \\
\hline $5 \mathrm{H}-3.17$ & 43.17 & 47.67 & 47.67 & 47.32 & 1.4973 & - & 30.01 & - \\
\hline $5 \mathrm{H}-3.28$ & 43.28 & 47.78 & 47.78 & 47.43 & 1.5010 & - & 30.00 & - \\
\hline $5 \mathrm{H}-3,47$ & 43,47 & 47.97 & 47.99 & 47.62 & 1.5073 & - & 30.00 & - \\
\hline $5 \mathrm{H}-3,62$ & 43.62 & 48.12 & 48.11 & 47.77 & 1.5123 & - & 30.00 & - \\
\hline $5 \mathrm{H}-3.77$ & 43.77 & 48.27 & 48.26 & 47.92 & 1.5173 & - & 30.00 & - \\
\hline $5 \mathrm{H}-3.92$ & 43,92 & 48.42 & 48.43 & 48.07 & 1.5223 & - & 30.00 & - \\
\hline $5 \mathrm{H}-3,107$ & 44.07 & 48.57 & 48.57 & 48.22 & 1.5273 & - & 30.00 & - \\
\hline $5 \mathrm{H}-3,122$ & 44.22 & 48.72 & 48.72 & 48.37 & 1.5321 & - & 31.54 & - \\
\hline $5 \mathrm{H}-3,137$ & 44.37 & 48.87 & 48.89 & 48.52 & 1.5368 & - & 31.79 & - \\
\hline $5 \mathrm{H}-4.2$ & 44.52 & 49.02 & 49.04 & 48.67 & 1.5415 & - & 31.79 & - \\
\hline $5 \mathrm{H}-4,17$ & 44.67 & 49.17 & 49.17 & 48.82 & 1.5462 & - & 31.79 & - \\
\hline $5 \mathrm{H}-4,28$ & 44.78 & 49.28 & 49.27 & 48.93 & 1.5497 & - & 31.79 & - \\
\hline $5 \mathrm{H}-4,47$ & 44.97 & 49.47 & 49.46 & 49.12 & 1.5557 & - & 31.79 & - \\
\hline $5 \mathrm{H}-4.62$ & 45.12 & 49.62 & 49.63 & 49.27 & 1.5604 & - & 31.79 & - \\
\hline $5 \mathrm{H}-4.77$ & 45.27 & 49.77 & 49,77 & 49.42 & 1.5651 & - & 31.79 & - \\
\hline $5 \mathrm{H}-4.92$ & 45.42 & 49.92 & 49.92 & 49.57 & 1.5703 & - & 28.85 & - \\
\hline $5 \mathrm{H}-4.107$ & 45.57 & 50.07 & 50.07 & 49.72 & 1.5758 & - & 27.18 & - \\
\hline $5 \mathrm{H}-4,122$ & 45.72 & 50.22 & 50.23 & 49.87 & 1.5813 & - & 27.18 & - \\
\hline $5 \mathrm{H}-4.137$ & 45.87 & 50.37 & 50.40 & 50.02 & 1.5869 & - & 27.18 & - \\
\hline $5 \mathrm{H}-5,2$ & 46.02 & 50.52 & 50.54 & 50.17 & 1.5924 & - & 27.18 & - \\
\hline $5 \mathrm{H}-5,17$ & 46.17 & 50.67 & 50.67 & 50.32 & 1.5979 & - & 27.18 & - \\
\hline $5 \mathrm{H}-5,28$ & 46.28 & 50.78 & 50.78 & 50.43 & 1.6020 & - & 27.18 & - \\
\hline $5 \mathrm{H}-5.47$ & 46.47 & 50.97 & 50.97 & 50.62 & 1.6092 & - & 26.39 & - \\
\hline $5 \mathrm{H}-5.62$ & 6.62 & 51.12 & 51.10 & 50.77 & 1.6151 & - & 25.38 & - \\
\hline $5 \mathrm{H}-5,77$ & 46.77 & 51.27 & 51.27 & 50.92 & 1.6210 & - & 25.38 & - \\
\hline $5 \mathrm{H}-5,92$ & 46.92 & 51.42 & 51.42 & 51.07 & 1.6269 & - & 25.38 & - \\
\hline $5 \mathrm{H}-5,107$ & 47.07 & 51.57 & 51.57 & 51.22 & 1.6328 & - & 25.38 & - \\
\hline $5 \mathrm{H}-5,122$ & 47.22 & 51.72 & 51.71 & 51.37 & 1.6387 & - & 25.38 & - \\
\hline $5 \mathrm{H}-5,137$ & 47.37 & 51.87 & 51.83 & 51.52 & 1.6446 & - & 25.38 & - \\
\hline $5 \mathrm{H}-6,2$ & 47.52 & 52.02 & 51.93 & 51.67 & 1.6505 & - & 25.38 & - \\
\hline $5 \mathrm{H}-6,17$ & 47.67 & 52.17 & 52.02 & 51.82 & 1.6564 & - & 25.38 & - \\
\hline $5 \mathrm{H}-6,28$ & 47.78 & 52.28 & 52.09 & 51.93 & 1.6608 & - & 25.38 & - \\
\hline $5 \mathrm{H}-6,47$ & 47.97 & 52.47 & 52.24 & 52.12 & 1.6682 & - & 25.38 & - \\
\hline $5 \mathrm{H}-6,64$ & 48.14 & 52.64 & 52.41 & 52.29 & 1.6749 & - & 25.38 & - \\
\hline $5 \mathrm{H}-6,77$ & 48.27 & 52.77 & 52.73 & 52.42 & 1.6801 & - & 25.38 & - \\
\hline $5 \mathrm{H}-6.92$ & 48.42 & 52.92 & 52.95 & 52.57 & 1.6860 & - & 25.38 & - \\
\hline $5 \mathrm{H}-6,107$ & 48.57 & 53.07 & 53.03 & 52.72 & 1.6919 & - & 25.38 & - \\
\hline $5 \mathrm{H}-6,122$ & 48.72 & 53.22 & 53.10 & 52.87 & 1.6972 & - & 28.16 & - \\
\hline $5 \mathrm{H}-6,137$ & 48.87 & 53.37 & 53.21 & 53.02 & 1.6987 & - & 97.62 & - \\
\hline $5 \mathrm{H}-7,2$ & 49.02 & 53.52 & 53.37 & 53.17 & 1.7003 & - & 97.62 & - \\
\hline $5 \mathrm{H}-7.17$ & 49.17 & 53.67 & 53.55 & 53.32 & 1.7018 & - & 97.62 & - \\
\hline $5 \mathrm{H}-7,28$ & 49.28 & 53.78 & 53.68 & 53.43 & 1.7029 & - & 97.62 & - \\
\hline $5 \mathrm{H}-7.47$ & 49.47 & 53.97 & 54.00 & 53.62 & 1.7049 & - & 97.62 & - \\
\hline $5 \mathrm{H}-7,64$ & 49.64 & 54.14 & 54.20 & 53.79 & 1.7066 & - & 97.62 & - \\
\hline 138-847D- & & & & & & & & \\
\hline $6 \mathrm{H}-3,32$ & 47.92 & 54.12 & 54.14 & 53.89 & 1.7077 & - & 97.62 & - \\
\hline $6 \mathrm{H}-3,47$ & 48.07 & 54.27 & 54.28 & 54.04 & 1.7092 & - & 97.62 & - \\
\hline $6 \mathrm{H}-3,62$ & 48.22 & 54.42 & 54.42 & 54.19 & 1.7107 & - & 97.62 & - \\
\hline $6 \mathrm{H}-3.77$ & 48.37 & 54.57 & 54.56 & 54.34 & 1.7123 & - & 97.62 & - \\
\hline $6 \mathrm{H}-3.92$ & 48.52 & 54.72 & 54.70 & 54.49 & 1.7138 & - & 97.62 & - \\
\hline $6 \mathrm{H}-3,107$ & 48.67 & 54.87 & 54.86 & 54.64 & 1.7153 & - & 97.62 & - \\
\hline $6 \mathrm{H}-3,122$ & 48.82 & 55.02 & 55.02 & 54.79 & 1.7169 & - & 97.62 & - \\
\hline $6 \mathrm{H}-3,137$ & 48.97 & 55.17 & 55.20 & 54.94 & 1.7203 & - & 43.43 & - \\
\hline $138-847 \mathrm{C}-$ & & & & & & & & \\
\hline $6 \mathrm{H}-1,17$ & 49.67 & 55.37 & 55.42 & 54.95 & 1.7209 & - & 17.19 & - \\
\hline $6 \mathrm{H}-1.30$ & 49.80 & 55.50 & 55.50 & 55.08 & 1.7285 & - & 17.19 & - \\
\hline $6 \mathrm{H}-1,47$ & 49.97 & 55.67 & 55.67 & 55.25 & 1.7384 & - & 17.19 & - \\
\hline $6 \mathrm{H}-1,62$ & 50.12 & 55.82 & 55.82 & 55.40 & 1.7471 & - & 17.19 & - \\
\hline $6 \mathrm{H}-1.77$ & 50.27 & 55.97 & 55.97 & 55.55 & 1.7534 & - & 23.75 & - \\
\hline $6 \mathrm{H}-1.92$ & 50.42 & 56.12 & 56.12 & 55.70 & 1.7585 & - & 29.34 & - \\
\hline $6 \mathrm{H}-1.107$ & 50.57 & 56.27 & 56.27 & 55.85 & 1.7636 & - & 29.34 & - \\
\hline $6 \mathrm{H}-1.122$ & 50.72 & 56.42 & 56.42 & 56.00 & 1.7687 & - & 29.34 & - \\
\hline $6 \mathrm{H}-1,137$ & 50.87 & 56.57 & 56.57 & 56.15 & 1.7739 & - & 29.34 & - \\
\hline $6 \mathrm{H}-2,2$ & 51.02 & 56.72 & 56.70 & 56.30 & 1.7790 & - & 29.34 & - \\
\hline $6 \mathrm{H}-2,17$ & 51.17 & 56.87 & 56.86 & 56.45 & 1.7841 & - & 29.34 & - \\
\hline $6 \mathrm{H}-2,30$ & 51.30 & 57.00 & 57.00 & 56.58 & 1.7885 & - & 29.34 & - \\
\hline $6 \mathrm{H}-2,47$ & 51.47 & 57.17 & 57.17 & 56.75 & 1.7943 & - & 29.34 & - \\
\hline $6 \mathrm{H}-2,58$ & 51.58 & 57.28 & 57.28 & 56.86 & 1.7981 & - & 29.34 & - \\
\hline $6 \mathrm{H}-2,77$ & 51.77 & 57.47 & 57.47 & 57.05 & 1.8045 & - & 29.34 & - \\
\hline $6 \mathrm{H}-2,92$ & 51.92 & 57.62 & 57.61 & 57.20 & 1.8096 & - & 29.34 & - \\
\hline $6 \mathrm{H}-2,107$ & 52.07 & 57.77 & 57.77 & 57.35 & 1.8130 & - & 44.02 & - \\
\hline $6 \mathrm{H}-2,122$ & 52.22 & 57.92 & 57.92 & 57.50 & 1.8158 & - & 53.81 & - \\
\hline $6 \mathrm{H}-2,137$ & 52.37 & 58.07 & 58.07 & 57.65 & 1.8186 & - & 53.81 & - \\
\hline $6 \mathrm{H}-3,2$ & 52.52 & 58.22 & 58.22 & 57.80 & 1.8214 & - & 53.81 & - \\
\hline $6 \mathrm{H}-3,17$ & 52.67 & 58.37 & 58.37 & 57.95 & 1.8242 & - & 53.81 & - \\
\hline $6 \mathrm{H}-3,29$ & 52.79 & 58.49 & 58.49 & 58.07 & 1.8264 & - & 53.81 & - \\
\hline $6 \mathrm{H}-3,47$ & 52.97 & 58.67 & 58.67 & 58.25 & 1.8298 & - & 53,81 & - \\
\hline $6 \mathrm{H}-3,62$ & 53.12 & 58.82 & 58.82 & 58.40 & 1.8325 & - & 54.46 & - \\
\hline $6 \mathrm{H}-3.77$ & 53.27 & 58.97 & 58.98 & 58.55 & 1.8351 & - & 57.21 & - \\
\hline $6 \mathrm{H}-3.92$ & 53.42 & 59.12 & 59.12 & 58.70 & 1.8378 & - & 57.21 & - \\
\hline
\end{tabular}


Table 1 (continued).

\begin{tabular}{|c|c|c|c|c|c|c|c|c|}
\hline \multirow[b]{2}{*}{$\begin{array}{l}\text { Core, section. } \\
\text { interval }(\mathrm{cm})\end{array}$} & \multirow[b]{2}{*}{$\begin{array}{l}\text { ODP } \\
\text { depth } \\
\text { (mbsf) }\end{array}$} & \multirow{2}{*}{$\begin{array}{l}\text { Shipboard } \\
\text { composite } \\
\text { depth } \\
\text { (mcd) }\end{array}$} & Revised & Composite & $\mathrm{Ag}$ & & Sediment: & ion rates \\
\hline & & & $\begin{array}{l}\text { composite } \\
\text { depth } \\
\text { (rmcd) }\end{array}$ & $\begin{array}{l}\text { depth, } \\
\text { this study } \\
\text { (Bmcd) }\end{array}$ & $\begin{array}{c}\text { Shackleton } \\
\text { age model } \\
(\mathrm{Ma})\end{array}$ & $\begin{array}{c}\text { Isotope } \\
\text { age model } \\
(\mathrm{Ma})\end{array}$ & $\begin{array}{c}\text { Shackleton } \\
\text { ages } \\
(\mathrm{m} / \mathrm{m} . \mathrm{y} .)\end{array}$ & $\begin{array}{c}\text { Isotope } \\
\text { ages } \\
\text { (m/m.y.) }\end{array}$ \\
\hline $6 \mathrm{H}-3,107$ & 53.57 & 59.27 & 59.27 & 58.85 & 1.8404 & - & 57.21 & - \\
\hline $6 \mathrm{H}-3,122$ & 53.72 & 59.42 & 59.44 & 59.00 & 1.8430 & - & 57.21 & - \\
\hline $6 \mathrm{H}-3,137$ & 53.87 & 59.57 & 59.57 & 59.15 & 1.8456 & - & 57.21 & - \\
\hline $6 \mathrm{H}-4.2$ & 54.02 & 59.72 & 59.70 & 59.30 & 1.8483 & - & 57.21 & - \\
\hline $6 \mathrm{H}-4,17$ & 54.17 & 59.87 & 59.86 & 59.45 & 1.8509 & - & 57.21 & - \\
\hline $6 \mathrm{H}-4.28$ & 54.28 & 59.98 & 59.99 & 59.56 & 1.8528 & - & 57.21 & - \\
\hline $6 \mathrm{H}-4.47$ & 54.47 & 60.17 & 60.16 & 59.75 & 1.8561 & - & 57.21 & - \\
\hline $6 \mathrm{H}-4,62$ & 54.62 & 60.32 & 60.32 & 59.90 & 1.8587 & - & 57.21 & - \\
\hline $6 \mathrm{H}-4,77$ & 54.77 & 60.47 & 60.47 & 60.05 & 1.8614 & - & 57.21 & - \\
\hline $6 \mathrm{H}-4,92$ & 54.92 & 60.62 & 60.62 & 60.20 & 1.8640 & - & 57.21 & - \\
\hline $6 \mathrm{H}-4,107$ & 55.07 & 60.77 & 60.77 & 60.35 & 1.8666 & - & 57.21 & - \\
\hline $6 \mathrm{H}-4,122$ & 55.22 & 60.92 & 60.92 & 60.50 & 1.8692 & - & 57.21 & - \\
\hline $6 \mathrm{H}-4,1.37$ & 55.37 & 61.07 & 61.07 & 60.65 & 1.8719 & - & 57.21 & - \\
\hline $6 \mathrm{H}-5,2$ & 55.52 & 61.22 & 61.22 & 60.80 & 1.8745 & - & 57.21 & - \\
\hline $6 \mathrm{H}-5,17$ & 55.67 & 61.37 & 61.37 & 60.95 & 1.8801 & - & 26.59 & - \\
\hline $6 \mathrm{H}-5,28$ & 55.78 & 61.48 & 61.48 & 61.06 & 1.8848 & - & 23.45 & - \\
\hline $6 \mathrm{H}-5,47$ & 55.97 & 61.67 & 61.67 & 61.25 & 1.8929 & - & 23.45 & - \\
\hline $6 \mathrm{H}-5,62$ & 56.12 & 61.82 & 61.82 & 61.40 & 1.8993 & - & 23.45 & - \\
\hline $6 \mathrm{H}-5,77$ & 56.27 & 61.97 & 61.97 & 61.55 & 1.9050 & - & 26.51 & - \\
\hline $6 \mathrm{H}-5,92$ & 56.42 & 62.12 & 62.12 & 61.70 & 1.9086 & - & 41.40 & - \\
\hline $6 \mathrm{H}-5,107$ & 56.57 & 62.27 & 62.27 & 61.85 & 1.9122 & - & 41.40 & - \\
\hline $6 \mathrm{H}-5,122$ & 56.72 & 62.42 & 62.42 & 62.00 & 1.9158 & - & 41.40 & - \\
\hline $6 \mathrm{H}-5,137$ & 56.87 & 62.57 & 62.57 & 62.15 & 1.9195 & - & 41.40 & - \\
\hline $6 \mathrm{H}-6,2$ & 57.02 & 62.72 & 62.72 & 62.30 & 1.9231 & - & 41.40 & - \\
\hline $6 \mathrm{H}-6.17$ & 57.17 & 62.87 & 62.87 & 62.45 & 1.9267 & - & 41.40 & - \\
\hline $6 \mathrm{H}-6,28$ & 57.28 & 62.98 & 62.98 & 62.56 & 1.9294 & - & 41.40 & - \\
\hline $6 \mathrm{H}-6,47$ & 57.47 & 63.17 & 63.17 & 62.75 & 1.9340 & - & 41.40 & - \\
\hline $6 \mathrm{H}-6,58$ & 57.58 & 63.28 & 63.28 & 62.86 & 1.9366 & - & 41.40 & - \\
\hline $6 \mathrm{H}-6.77$ & 57.77 & 63.47 & 63.47 & 63.05 & 1.9412 & - & 41.40 & - \\
\hline $6 \mathrm{H}-6,92$ & 57.92 & 63.62 & 63.63 & 63.20 & 1.9448 & - & 41.40 & - \\
\hline $6 \mathrm{H}-6,107$ & 58.07 & 63.77 & 63.76 & 63.35 & 1.9477 & - & 52.01 & - \\
\hline $6 \mathrm{H}-6,122$ & 58.22 & 63.92 & 63.92 & 63.50 & 1.9495 & - & 84.55 & - \\
\hline $6 \mathrm{H}-6,137$ & 58.37 & 64.07 & 64.08 & 63.65 & 1.9513 & - & 84.55 & - \\
\hline $6 \mathrm{H}-7.2$ & 58.52 & 64.22 & 64.21 & 63.80 & 1.9530 & - & 84.55 & - \\
\hline $6 \mathrm{H}-7,17$ & 58.67 & 64.37 & 64.37 & 63.95 & 1.9548 & - & 84.55 & - \\
\hline 138-847D- & & & & & & & & \\
\hline $7 \mathrm{H}-3,17$ & 57.27 & 64.47 & 64.45 & 64.05 & 1.9560 & - & 84.55 & - \\
\hline $7 \mathrm{H}-3,32$ & 57.42 & 64.62 & 64.64 & 64.20 & 1.9578 & - & 84.55 & - \\
\hline $7 \mathrm{H}-3,47$ & 57.57 & 64.77 & 64.83 & 64.35 & 1.9619 & - & 36.49 & - \\
\hline $7 \mathrm{H}-3,62$ & 57.72 & 64.92 & 64.95 & 64.50 & 1.9663 & - & 33.56 & - \\
\hline $7 \mathrm{H}-3,77$ & 57.87 & 65.07 & 65.07 & 64.65 & 1.9708 & - & 33.56 & - \\
\hline $7 \mathrm{H}-3,92$ & 58.02 & 65.22 & 65.22 & 64.80 & 1.9753 & - & 33.56 & - \\
\hline $7 \mathrm{H}-3,107$ & 58.17 & 65.37 & 65.37 & 64.95 & 1.9798 & - & 33.56 & - \\
\hline $7 \mathrm{H}-3,122$ & 58.32 & 65.52 & 65.50 & 65.10 & 1.9842 & - & 33.56 & - \\
\hline $7 \mathrm{H}-3,137$ & 58.47 & 65.67 & 65.66 & 65.25 & 1.9887 & - & 33.56 & - \\
\hline $7 \mathrm{H}-4,2$ & 58.62 & 65.82 & 65.83 & 65.40 & 1.9932 & - & 33.56 & - \\
\hline $7 \mathrm{H}-4,17$ & 58.77 & 65.97 & 65.98 & 65.55 & 1.9976 & - & 33.56 & - \\
\hline $7 \mathrm{H}-4,32$ & 58.92 & 66.12 & 66.12 & 65.70 & 2.0021 & - & 33.56 & - \\
\hline $7 \mathrm{H}-4,47$ & 59.07 & 66.27 & 66.27 & 65.85 & 2.0065 & - & 34.31 & - \\
\hline $7 \mathrm{H}-4,62$ & 59.22 & 66.42 & 66.42 & 66.00 & 2.0108 & - & 34.50 & - \\
\hline $7 \mathrm{H}-4,77$ & 59.37 & 66.57 & 66.57 & 66.15 & 2.0152 & - & 34.50 & - \\
\hline $7 \mathrm{H}-4,92$ & 59.52 & 66.72 & 66.71 & 66.30 & 2.0195 & - & 34.50 & - \\
\hline $138-847 \mathrm{C}-$ & & & & & & & & \\
\hline $7 \mathrm{H}-1,17$ & 59.17 & 66.97 & 66.73 & 66.32 & 2.0201 & - & 34.50 & - \\
\hline $7 \mathrm{H}-1,30$ & 59.30 & 67.10 & 66.86 & 66.45 & 2.0235 & - & 38.62 & - \\
\hline $7 \mathrm{H}-1,47$ & 59.47 & 67.27 & 67.02 & 66.62 & 2.0261 & - & 64.21 & - \\
\hline $7 \mathrm{H}-1,62$ & 59.62 & 67.42 & 67.16 & 66.77 & 2.0285 & - & 64.21 & - \\
\hline $7 \mathrm{H}-1,77$ & 59.77 & 67.57 & 67.31 & 66.92 & 2.0308 & - & 64.21 & - \\
\hline $7 \mathrm{H}-1,92$ & 59.92 & 67.72 & 67.48 & 67.07 & 2.0331 & - & 64.21 & - \\
\hline $7 \mathrm{H}-1,107$ & 60.07 & 67.87 & 67.68 & 67.22 & 2.0355 & - & 64.21 & - \\
\hline $7 \mathrm{H}-1,122$ & 60.22 & 68.02 & 67.87 & 67.37 & 2.0378 & - & 64.21 & - \\
\hline $7 \mathrm{H}-1,137$ & 60.37 & 68.17 & 68.03 & 67.52 & 2.0401 & - & 64.21 & - \\
\hline $7 \mathrm{H}-2,2$ & 60.52 & 68.32 & 68.18 & 67.67 & 2.0428 & - & 55.98 & - \\
\hline $7 \mathrm{H}-2,17$ & 60.67 & 68.47 & 68.35 & 67.82 & 2.0469 & - & 37.00 & - \\
\hline $7 \mathrm{H}-2,30$ & 60.80 & 68.60 & 68.51 & 67.95 & 2.0504 & - & 37.00 & - \\
\hline $7 \mathrm{H}-2,47$ & 60.97 & 68.77 & 68.74 & 68.12 & 2.0555 & - & 33.12 & - \\
\hline $7 \mathrm{H}-2,64$ & 61.14 & 68.94 & 68.95 & 68.29 & 2.0609 & - & 31.33 & - \\
\hline $7 \mathrm{H}-2.77$ & 61.27 & 69.07 & 69.08 & 68.42 & 2.0651 & - & 31.33 & - \\
\hline $7 \mathrm{H}-2,92$ & 61.42 & 69.22 & 69.22 & 68.57 & 2.0699 & - & 31.33 & - \\
\hline $7 \mathrm{H}-2,107$ & 61.57 & 69.37 & 69.37 & 68.72 & 2.0747 & - & 31.33 & - \\
\hline $7 \mathrm{H}-2,122$ & 61.72 & 69.52 & 69.52 & 68.87 & 2.0794 & - & 31.33 & - \\
\hline $7 \mathrm{H}-2,137$ & 61.87 & 69.67 & 69.67 & 69.02 & 2.0842 & - & 31.33 & - \\
\hline $7 \mathrm{H}-3,2$ & 62.02 & 69.82 & 69.83 & 69.17 & 2.0890 & - & 31.33 & - \\
\hline $7 \mathrm{H}-3,17$ & 62.17 & 69.97 & 69.98 & 69.32 & 2.0938 & - & 31.33 & - \\
\hline $7 \mathrm{H}-3.30$ & 62.30 & 70.10 & 70.10 & 69.45 & 2.0983 & - & 28.86 & - \\
\hline $7 \mathrm{H}-3,47$ & 62.47 & 70.27 & 70.27 & 69.62 & 2.1058 & - & 22.86 & - \\
\hline $7 \mathrm{H}-3,62$ & 62.62 & 70.42 & 70.42 & 69.77 & 2.1123 & - & 22.86 & - \\
\hline $7 \mathrm{H}-3,77$ & 62.77 & 70.57 & 70.57 & 69.92 & 2.1188 & - & 23.17 & - \\
\hline $7 \mathrm{H}-3,92$ & 62.92 & 70.72 & 70.72 & 70.07 & 2.1247 & - & 25.45 & - \\
\hline $7 \mathrm{H}-3,107$ & 63.07 & 70.87 & 70.87 & 70.22 & 2.1303 & - & 26.71 & - \\
\hline $7 \mathrm{H}-3,122$ & 63.22 & 71.02 & 71.02 & 70.37 & 2.1351 & - & 30.91 & - \\
\hline $7 \mathrm{H}-3.137$ & 63.37 & 71.17 & 71.17 & 70.52 & 2.1400 & - & 30.91 & - \\
\hline
\end{tabular}


Table 1 (continued).

\begin{tabular}{|c|c|c|c|c|c|c|c|c|}
\hline \multirow[b]{2}{*}{$\begin{array}{l}\text { Core, section, } \\
\text { interval }(\mathrm{cm})\end{array}$} & \multirow[b]{2}{*}{$\begin{array}{c}\text { ODP } \\
\text { depth } \\
\text { (mbsf) }\end{array}$} & \multirow[b]{2}{*}{$\begin{array}{l}\text { Shipboard } \\
\text { composite } \\
\text { depth } \\
\text { (mcd) }\end{array}$} & & & As & & Sediment & ion rates \\
\hline & & & $\begin{array}{l}\text { composite } \\
\text { depth } \\
\text { (rmcd) }\end{array}$ & $\begin{array}{l}\text { depth. } \\
\text { this study } \\
\text { (Bmcd) }\end{array}$ & $\begin{array}{l}\text { Shackleton } \\
\text { age model } \\
\text { (Ma) }\end{array}$ & $\begin{array}{c}\text { Isotope } \\
\text { age model } \\
(\mathrm{Ma})\end{array}$ & $\begin{array}{c}\text { Shackleton } \\
\text { ages } \\
(\mathrm{m} / \mathrm{m} . \mathrm{y} .)\end{array}$ & $\begin{array}{c}\text { Isotope } \\
\text { ages } \\
(\mathrm{m} / \mathrm{m} . \mathrm{y} .)\end{array}$ \\
\hline $7 \mathrm{H}-4,2$ & 63.52 & 71.32 & 71.32 & 70.67 & 2.1450 & - & 30.28 & - \\
\hline $7 \mathrm{H}-4,17$ & 63.67 & 71.47 & 71.47 & 70.82 & 2.1499 & - & 30.28 & - \\
\hline $7 \mathrm{H}-4,30$ & 63.80 & 71.60 & 71.60 & 70.95 & 2.1542 & - & 30.28 & - \\
\hline $7 \mathrm{H}-4.47$ & 63.97 & 71.77 & 71.77 & 71.12 & 2.1598 & - & 30.28 & - \\
\hline $7 \mathrm{H}-4.62$ & 64.12 & 71.92 & 71.92 & 71.27 & 2.1648 & - & 30.28 & - \\
\hline $7 \mathrm{H}-4.77$ & 64.27 & 72.07 & 72.07 & 71.42 & 2.1697 & - & 30.28 & - \\
\hline $7 \mathrm{H}-4,92$ & 64.42 & 72.22 & 72.22 & 71.57 & 2.1747 & - & 30.28 & - \\
\hline $7 \mathrm{H}-4,107$ & 64.57 & 72.37 & 72.37 & 71.72 & 2.1796 & - & 30.28 & - \\
\hline $7 \mathrm{H}-4,122$ & 64.72 & 72.52 & 72.52 & 71.87 & 2.1846 & - & 30.28 & - \\
\hline $7 \mathrm{H}-4,137$ & 64.87 & 72.67 & 72.67 & 72.02 & 2.1895 & - & 30.28 & - \\
\hline $7 \mathrm{H}-5.2$ & 65.02 & 72.82 & 72.82 & 72.17 & 2.1945 & - & 30.28 & - \\
\hline $7 \mathrm{H}-5,17$ & 65.17 & 72.97 & 72.97 & 72.32 & 2.1994 & - & 30.28 & - \\
\hline $7 \mathrm{H}-5,30$ & 65.30 & 73.10 & 73.10 & 72.45 & 2.2037 & - & 30.28 & - \\
\hline $7 \mathrm{H}-5,47$ & 65.47 & 73.27 & 73.27 & 72.62 & 2.2093 & - & 30.28 & - \\
\hline $7 \mathrm{H}-5,62$ & 65.62 & 73.42 & 73.42 & 72.77 & 2.2141 & - & 31.58 & - \\
\hline $7 \mathrm{H}-5,77$ & 65.77 & 73.57 & 73.57 & 72.92 & 2.2187 & - & 32.27 & - \\
\hline $7 \mathrm{H}-5.92$ & 65.92 & 73.72 & 73.72 & 73.07 & 2.2234 & - & 32.27 & - \\
\hline $7 \mathrm{H}-5,107$ & 66.07 & 73.87 & 73.87 & 73.22 & 2.2280 & - & 32.27 & - \\
\hline $7 \mathrm{H}-5,122$ & 66.22 & 74.02 & 74.02 & 73.37 & 2.2327 & - & 32.27 & - \\
\hline $7 \mathrm{H}-5,137$ & 66.37 & 74.17 & 74.17 & 73.52 & 2.2362 & - & 42.64 & - \\
\hline $7 \mathrm{H}-6,2$ & 66.52 & 74.32 & 74.32 & 73.67 & 2.2396 & - & 43.64 & - \\
\hline $7 \mathrm{H}-6,17$ & 66.67 & 74.47 & 74.47 & 73.82 & 2.2431 & - & 43.64 & - \\
\hline $7 \mathrm{H}-6,30$ & 66.80 & 74.60 & 74.60 & 73.95 & 2.2461 & - & 43.64 & - \\
\hline $7 \mathrm{H}-6.47$ & 66.97 & 74.77 & 74.78 & 74.12 & 2.2500 & - & 43.64 & - \\
\hline $7 \mathrm{H}-6,64$ & 67.14 & 74.94 & 74.94 & 74.29 & 2.2539 & - & 43.64 & - \\
\hline $7 \mathrm{H}-6,77$ & 67.27 & 75.07 & 75.07 & 74.42 & 2.2585 & - & 27.75 & - \\
\hline $7 \mathrm{H}-6,92$ & 67.42 & 75.22 & 75.21 & 74.57 & 2.2652 & - & 22.61 & - \\
\hline $7 \mathrm{H}-6,107$ & 67.57 & 75.37 & 75.36 & 74.72 & 2.2718 & - & 22.61 & - \\
\hline $7 \mathrm{H}-6,122$ & 67.72 & 75.52 & 75.52 & 74.87 & 2.2784 & - & 22.78 & - \\
\hline $7 \mathrm{H}-6,137$ & 67.87 & 75.67 & 75.68 & 75.02 & 2.2843 & - & 25.56 & - \\
\hline $7 \mathrm{H}-7.2$ & 68.02 & 75.82 & 75.83 & 75.17 & 2.2901 & - & 25.56 & - \\
\hline $7 \mathrm{H}-7,17$ & 68.17 & 75.97 & 75.97 & 75.32 & 2.2960 & - & 25.56 & - \\
\hline $7 \mathrm{H}-7,30$ & 68.30 & 76.10 & 76.08 & 75.45 & 2.3011 & - & 25.56 & - \\
\hline 138-847D- & & & & & & & & \\
\hline $8 \mathrm{H}-3,122$ & 67.82 & 76.12 & 76.13 & 75.55 & 2.3050 & - & 25.56 & - \\
\hline $8 \mathrm{H}-3,137$ & 67.97 & 76.27 & 76.26 & 75.70 & 2.3100 & - & 30.23 & - \\
\hline $8 \mathrm{H}-4,2$ & 68.12 & 76.42 & 76.40 & 75.85 & 2.3149 & - & 30.23 & - \\
\hline $8 \mathrm{H}-4,17$ & 68.27 & 76.57 & 76.56 & 76.00 & 2.3199 & - & 30.23 & - \\
\hline $8 \mathrm{H}-4,32$ & 68.42 & 76.72 & 76.73 & 76.15 & 2.3248 & - & 30.23 & - \\
\hline $8 \mathrm{H}-4,47$ & 68.57 & 76.87 & 76.88 & 76.30 & 2.3298 & - & 30.23 & - \\
\hline $8 \mathrm{H}-4.62$ & 68.72 & 77.02 & 77.02 & 76.45 & 2.3348 & - & 30.23 & - \\
\hline $8 \mathrm{H}-4.77$ & 68.87 & 77.17 & 77.17 & 76.60 & 2.3397 & - & 30.23 & - \\
\hline $8 \mathrm{H}-4.92$ & 69.02 & 77.32 & 77.32 & 76.75 & 2.3447 & - & 30.23 & - \\
\hline $8 \mathrm{H}-4,107$ & 69.17 & 77.47 & 77.48 & 76.90 & 2.3493 & - & 32.58 & - \\
\hline $8 \mathrm{H}-4,122$ & 69.32 & 77.62 & 77.62 & 77.05 & 2.3532 & - & 38.57 & - \\
\hline $8 \mathrm{H}-4.137$ & 69.47 & 77.77 & 77.77 & 77.20 & 2.3571 & - & 38.57 & - \\
\hline $8 \mathrm{H}-5.2$ & 69.62 & 77.92 & 77.91 & 77.35 & 2.3610 & - & 38.57 & - \\
\hline $8 \mathrm{H}-5,17$ & 69.77 & 78.07 & 78.06 & 77.50 & 2.3649 & - & 38.57 & - \\
\hline $8 \mathrm{H}-5,32$ & 69.92 & 78.22 & 78.22 & 77.65 & 2.3687 & - & 38.57 & - \\
\hline $138-847 \mathrm{C}$ - & & & & & & & & \\
\hline $8 \mathrm{H}-2.2$ & 70.02 & 78.22 & 78.26 & 77.75 & 2.3713 & - & 38.57 & - \\
\hline $8 \mathrm{H}-2,17$ & 70.17 & 78.37 & 78.48 & 77.90 & 2.3752 & - & 38.57 & - \\
\hline $8 \mathrm{H}-2,32$ & 70.32 & 78.52 & 78.61 & 78.05 & 2.3791 & - & 38.57 & - \\
\hline $8 \mathrm{H}-2.47$ & 70.47 & 78.67 & 78.72 & 78.20 & 2.3830 & - & 38.57 & - \\
\hline $8 \mathrm{H}-2,62$ & 70.62 & 78.82 & 78.81 & 78.35 & 2.3869 & - & 38.57 & - \\
\hline $8 \mathrm{H}-2.78$ & 70.78 & 78.98 & 78.94 & 78.51 & 2.3916 & - & 34.18 & - \\
\hline $8 \mathrm{H}-2.92$ & 70.92 & 79.12 & 79.07 & 78.65 & 2.3971 & - & 25.47 & - \\
\hline $8 \mathrm{H}-2,107$ & 71.07 & 79.27 & 79.22 & 78.80 & 2.4030 & - & 25.47 & - \\
\hline $8 \mathrm{H}-2,122$ & 71.22 & 79.42 & 79.37 & 78.95 & 2.4088 & - & 25.47 & - \\
\hline $8 \mathrm{H}-2,137$ & 71.37 & 79.57 & 79.51 & 79.10 & 2.4147 & - & 25.47 & - \\
\hline $8 \mathrm{H}-3,2$ & 71.52 & 79.72 & 79.66 & 79.25 & 2.4206 & - & 25.47 & - \\
\hline $8 \mathrm{H}-3,17$ & 71.67 & 79.87 & 79.79 & 79.40 & 2.4244 & - & 39.36 & - \\
\hline $8 \mathrm{H}-3,32$ & 71.82 & 80.02 & 79.89 & 79.55 & 2.4276 & - & 47.19 & - \\
\hline $8 \mathrm{H}-3,47$ & 71.97 & 80.17 & 79.99 & 79.70 & 2.4308 & - & 47.19 & - \\
\hline $8 \mathrm{H}-3,62$ & 72.12 & 80.32 & 80.13 & 79.85 & 2.4340 & - & 47.19 & - \\
\hline $8 \mathrm{H}-3,78$ & 72.28 & 80.48 & 80.35 & 80.01 & 2.4374 & - & 47.19 & - \\
\hline $8 \mathrm{H}-3,92$ & 72.42 & 80.62 & 80.56 & 80.15 & 2.4403 & - & 47.98 & - \\
\hline $8 \mathrm{H}-3,107$ & 72.57 & 80.77 & 80.71 & 80.30 & 2.4434 & - & 48.21 & - \\
\hline $8 \mathrm{H}-3,122$ & 72.72 & 80.92 & 80.83 & 80.45 & 2.4465 & - & 48.21 & - \\
\hline $8 \mathrm{H}-3,137$ & 72.87 & 81.07 & 80.98 & 80.60 & 2.4496 & - & 48.21 & - \\
\hline $8 \mathrm{H}-4.2$ & 73.02 & 81.22 & 81.19 & 80.75 & 2.4527 & - & 48.21 & - \\
\hline $8 \mathrm{H}-4,17$ & 73.17 & 81.37 & 81.38 & 80.90 & 2.4558 & - & 48.21 & - \\
\hline $8 \mathrm{H}-4,32$ & 73.32 & 81.52 & 81.52 & 81.05 & 2.4590 & - & 48.21 & - \\
\hline $8 \mathrm{H}-4,47$ & 73.47 & 81.67 & 81.65 & 81.20 & 2.4621 & - & 48.21 & - \\
\hline $8 \mathrm{H}-4,62$ & 73.62 & 81.82 & 81.81 & 81.35 & 2.4652 & - & 48.21 & - \\
\hline $8 \mathrm{H}-4.78$ & 73.78 & 81.98 & 81.99 & 81.51 & 2.4685 & - & 48.21 & - \\
\hline $8 \mathrm{H}-4.92$ & 73.92 & 82.12 & 82.13 & 81.65 & 2.4714 & - & 48.21 & - \\
\hline $8 \mathrm{H}-4,107$ & 74.07 & 82.27 & 82.27 & 81.80 & 2.4745 & - & 48.21 & - \\
\hline $8 \mathrm{H}-4,122$ & 74.22 & 82,42 & 82.42 & 81.95 & 2.4781 & - & 41.57 & - \\
\hline $8 \mathrm{H}-4.137$ & 74.37 & 82.57 & 82.58 & 82.10 & 2.4837 & - & 26.82 & - \\
\hline $8 \mathrm{H}-5.2$ & 74.52 & 82.72 & 82.73 & 82.25 & 2.4893 & - & 26.82 & - \\
\hline $8 \mathrm{H}-5,17$ & 74.67 & 82.87 & 82.87 & 82.40 & 2.4949 & - & 26.82 & - \\
\hline
\end{tabular}


Table 1 (continued).

\begin{tabular}{|c|c|c|c|c|c|c|c|c|}
\hline \multirow[b]{2}{*}{$\begin{array}{l}\text { Core, section, } \\
\text { interval }(\mathrm{cm})\end{array}$} & \multirow[b]{2}{*}{$\begin{array}{c}\text { ODP } \\
\text { depth } \\
\text { (mbsf) }\end{array}$} & \multirow{2}{*}{$\begin{array}{c}\text { Shipboard } \\
\text { composite } \\
\text { depth } \\
\text { (mcd) }\end{array}$} & Revised & Composite & $\mathrm{As}_{\mathrm{s}}$ & & Sediment: & ion rates \\
\hline & & & $\begin{array}{l}\text { composite } \\
\text { depth } \\
\text { (rmcd) }\end{array}$ & $\begin{array}{l}\text { depth, } \\
\text { this study } \\
\text { (Bmcd) }\end{array}$ & $\begin{array}{c}\text { Shackleton } \\
\text { age model } \\
\text { (Ma) }\end{array}$ & $\begin{array}{c}\text { Isotope } \\
\text { age model } \\
(\mathrm{Ma})\end{array}$ & $\begin{array}{l}\text { Shackleton } \\
\text { ages } \\
(\mathrm{m} / \mathrm{m} . \mathrm{y} .)\end{array}$ & $\begin{array}{c}\text { Isotope } \\
\text { ages } \\
\text { (m/m.y.) }\end{array}$ \\
\hline $8 \mathrm{H}-5,32$ & 74.82 & 83.02 & 83.01 & 82.55 & 2.5005 & - & 26.82 & - \\
\hline $8 \mathrm{H}-5,47$ & 74.97 & 83.17 & 83.17 & 82.70 & 2.5061 & - & 26.82 & - \\
\hline $8 H-5,62$ & 75.12 & 83.32 & 83.33 & 82.85 & 2.5117 & - & 26.82 & - \\
\hline $8 \mathrm{H}-5,78$ & 75.28 & 83.48 & 83.49 & 83.01 & 2.5176 & - & 26.82 & - \\
\hline $8 \mathrm{H}-5.92$ & 75.42 & 83.62 & 83.61 & 83.15 & 2.5223 & - & 29.81 & - \\
\hline $8 \mathrm{H}-5,107$ & 75.57 & 83.77 & 83.75 & 83.30 & 2.5264 & - & 37.31 & - \\
\hline $8 \mathrm{H}-5,122$ & 75.72 & 83.92 & 83.91 & 83.45 & 2.5304 & - & 37.31 & - \\
\hline $8 \mathrm{H}-5,137$ & 75.87 & 84.07 & 84.08 & 83.60 & 2.5344 & - & 37.31 & - \\
\hline $8 \mathrm{H}-6,2$ & 76.02 & 84.22 & 84.22 & 83.75 & 2.5384 & - & 37.31 & - \\
\hline $8 \mathrm{H}-6,17$ & 76.17 & 84.37 & 84.35 & 83.90 & 2.5424 & - & 37.31 & - \\
\hline $8 \mathrm{H}-6,32$ & 76.32 & 84.52 & 84.47 & 84.05 & 2.5465 & - & 37.31 & - \\
\hline $8 \mathrm{H}-6,47$ & 76.47 & 84.67 & 84.60 & 84.20 & 2.5531 & - & 22.65 & - \\
\hline $8 \mathrm{H}-6,62$ & 76.62 & 84.82 & 84.72 & 84.35 & 2.5601 & - & 21.36 & - \\
\hline $8 \mathrm{H}-6,78$ & 76.78 & 84.98 & 84.83 & 84.51 & 2.5676 & - & 21.36 & - \\
\hline $8 \mathrm{H}-6,92$ & 76.92 & 85.12 & 84.92 & 84.65 & 2.5713 & - & 37.79 & - \\
\hline 138-847D- & & & & & & & & \\
\hline $9 \mathrm{H}-3,32$ & 76.42 & 85.12 & 85.10 & 84.85 & 2.5755 & - & 47.83 & - \\
\hline $9 \mathrm{H}-3,47$ & 76.57 & 85.27 & 85.26 & 85.00 & 2.5786 & - & 47.83 & - \\
\hline $9 \mathrm{H}-3,62$ & 76.72 & 85.42 & 85.42 & 85.15 & 2.5818 & - & 47.83 & - \\
\hline $9 \mathrm{H}-3,77$ & 76.87 & 85.57 & 85.57 & 85.30 & 2.5849 & - & 47.83 & - \\
\hline $9 \mathrm{H}-3,92$ & 77.02 & 85.72 & 85.73 & 85.45 & 2.5880 & - & 47.83 & - \\
\hline $9 \mathrm{H}-3,107$ & 77.17 & 85.87 & 85.87 & 85.60 & 2.5912 & - & 47.83 & - \\
\hline $9 \mathrm{H}-3,122$ & 77.32 & 86.02 & 86.02 & 85.75 & 2.5948 & - & 40.72 & - \\
\hline $9 \mathrm{H}-3,137$ & 77.47 & 86.17 & 86.17 & 85.90 & 2.5987 & - & 38.64 & - \\
\hline $9 \mathrm{H}-4,2$ & 77.62 & 86.32 & 86.32 & 86.05 & 2.6026 & - & 38.64 & - \\
\hline $9 \mathrm{H}-4,17$ & 77.77 & 86.47 & 86.47 & 86.20 & 2.6065 & - & 38.64 & - \\
\hline $9 \mathrm{H}-4,32$ & 77.92 & 86.62 & 86.63 & 86.35 & 2.6104 & - & 38.64 & - \\
\hline $9 \mathrm{H}-4,47$ & 78.07 & 86.77 & 86.78 & 86.50 & 2.6145 & - & 36.73 & - \\
\hline $9 \mathrm{H}-4,62$ & 78.22 & 86.92 & 86.92 & 86.65 & 2.6214 & - & 21.74 & - \\
\hline $9 \mathrm{H}-4,77$ & 78.37 & 87.07 & 87.07 & 86.80 & 2.6283 & - & 21.74 & - \\
\hline $9 \mathrm{H}-4,92$ & 78.52 & 87.22 & 87.21 & 86.95 & 2.6352 & - & 21.74 & - \\
\hline $9 \mathrm{H}-4,107$ & 78.67 & 87.37 & 87.36 & 87.10 & 2.6404 & - & 28.40 & - \\
\hline $9 \mathrm{H}-4,122$ & 78.82 & 87.52 & 87.51 & 87.25 & 2.6451 & - & 31.96 & - \\
\hline $9 \mathrm{H}-4,137$ & 78.97 & 87.67 & 87.66 & 87.40 & 2.6498 & - & 31.96 & - \\
\hline $9 \mathrm{H}-5,2$ & 79.12 & 87.82 & 87.82 & 87.55 & 2.6545 & - & 31.96 & - \\
\hline $9 \mathrm{H}-5,17$ & 79.27 & 87.97 & 87.97 & 87.70 & 2.6592 & - & 31.96 & - \\
\hline 138-847C- & & & & & & & & \\
\hline $9 \mathrm{H}-1,32$ & 78.32 & 88.22 & 88.12 & 87.87 & 2.6645 & - & 31.96 & - \\
\hline $9 \mathrm{H}-\mathrm{I}, 47$ & 78.47 & 88.37 & 88.21 & 88.02 & 2.6692 & - & 31.96 & - \\
\hline $9 \mathrm{H}-1,62$ & 78.62 & 88.52 & 88.37 & 88.17 & 2.6739 & - & 31.96 & - \\
\hline $9 \mathrm{H}-1,77$ & 78.77 & 88.67 & 88.61 & 88.32 & 2.6786 & - & 31.96 & - \\
\hline $9 \mathrm{H}-1,92$ & 78.92 & 88.82 & 88.85 & 88.47 & 2.6834 & - & 31.58 & - \\
\hline $9 \mathrm{H}-1,107$ & 79.07 & 88.97 & 89.02 & 88.62 & 2.6889 & - & 27.08 & - \\
\hline $9 \mathrm{H}-1,122$ & 79.22 & 89.12 & 89.14 & 88.77 & 2.6944 & - & 27.08 & - \\
\hline $9 \mathrm{H}-1,137$ & 79.37 & 89.27 & 89.27 & 88.92 & 2.7000 & - & 27.08 & - \\
\hline $9 \mathrm{H}-2,2$ & 79.52 & 89.42 & 89.42 & 89.07 & 2.7055 & - & 27.08 & - \\
\hline $9 \mathrm{H}-2,17$ & 79.67 & 89.57 & 89.61 & 89.22 & 2.7133 & - & 19.32 & - \\
\hline $9 \mathrm{H}-2,32$ & 79.82 & 89.72 & 89.79 & 89.37 & 2.7219 & - & 17.50 & - \\
\hline $9 \mathrm{H}-2,47$ & 79.97 & 89.87 & 89.91 & 89.52 & 2.7295 & - & 19.64 & - \\
\hline $9 \mathrm{H}-2,62$ & 80.12 & 90.02 & 90.00 & 89.67 & 2.7325 & - & 50.60 & - \\
\hline $9 \mathrm{H}-2,77$ & 80.27 & 90.17 & 90.10 & 89.82 & 2.7354 & - & 50.60 & - \\
\hline $9 \mathrm{H}-2,92$ & 80.42 & 90.32 & 90.27 & 89.97 & 2.7384 & - & 50.60 & - \\
\hline $9 \mathrm{H}-2,107$ & 80.57 & 90.47 & 90.47 & 90.12 & 2.7414 & - & 50.60 & - \\
\hline $9 \mathrm{H}-2,122$ & 80.72 & 90.62 & 90.62 & 90.27 & 2.7443 & - & 50.60 & - \\
\hline $9 \mathrm{H}-2,137$ & 80.87 & 90.77 & 90.77 & 90.42 & 2.7473 & - & 50.60 & - \\
\hline $9 \mathrm{H}-3,2$ & 81.02 & 90.92 & 90.92 & 90.57 & 2.7502 & - & 50.60 & - \\
\hline $9 \mathrm{H}-3,17$ & 81.17 & 91.07 & 91.07 & 90.72 & 2.7532 & - & 50.60 & - \\
\hline $9 \mathrm{H}-3,32$ & 81.32 & 91.22 & 91.22 & 90.87 & 2.7607 & - & 19.97 & - \\
\hline $9 \mathrm{H}-3,47$ & 81.47 & 91.37 & 91.37 & 91.02 & 2.7699 & - & 16.36 & - \\
\hline $9 \mathrm{H}-3,62$ & 81.62 & 91.52 & 91.52 & 91.17 & 2.7776 & - & 19.55 & - \\
\hline $9 \mathrm{H}-3,77$ & 81.77 & 91.67 & 91.67 & 91.32 & 2.7823 & - & 32.00 & - \\
\hline $9 \mathrm{H}-3,92$ & 81.92 & 91.82 & 91.82 & 91.47 & 2.7865 & - & 34.92 & - \\
\hline $9 \mathrm{H}-3,107$ & 82.07 & 91.97 & 91.97 & 91.62 & 2.7893 & - & 55.00 & - \\
\hline $9 \mathrm{H}-3,122$ & 82.22 & 92.12 & 92.12 & 91.77 & 2.7920 & - & 55.00 & - \\
\hline $9 \mathrm{H}-3,137$ & 82.37 & 92.27 & 92.27 & 91.92 & 2.7947 & - & 55.00 & - \\
\hline $9 \mathrm{H}-4,2$ & 82.52 & 92.42 & 92.42 & 92.07 & 2.7975 & - & 55.00 & - \\
\hline $9 \mathrm{H}-4,17$ & 82.67 & 92.57 & 92.57 & 92.22 & 2.8010 & - & 42.31 & - \\
\hline $9 \mathrm{H}-4,32$ & 82.82 & 92.72 & 92.72 & 92.37 & 2.8048 & - & 40.00 & - \\
\hline $9 \mathrm{H}-4,47$ & 82.97 & 92.87 & 92.87 & 92.52 & 2.8085 & - & 40.00 & - \\
\hline $9 \mathrm{H}-4,62$ & 83.12 & 93.02 & 93.02 & 92.67 & 2.8123 & - & 40.00 & - \\
\hline $9 \mathrm{H}-4,77$ & 83.27 & 93.17 & 93.17 & 92.82 & 2.8160 & - & 40.00 & - \\
\hline $9 \mathrm{H}-4,92$ & 83.42 & 93.32 & 93.32 & 92.97 & 2.8198 & - & 40.00 & - \\
\hline $9 \mathrm{H}-4,107$ & 83.57 & 93.47 & 93.47 & 93.12 & 2.8235 & - & 40.00 & - \\
\hline $9 \mathrm{H}-4,122$ & 83.72 & 93.62 & 93.61 & 93.27 & 2.8273 & - & 40.00 & - \\
\hline $9 \mathrm{H}-4,137$ & 83.87 & 93.77 & 93.77 & 93.42 & 2.8310 & - & 40.00 & - \\
\hline $9 \mathrm{H}-5.2$ & 84.02 & 93.92 & 93.92 & 93.57 & 2.8348 & - & 40.00 & - \\
\hline $9 \mathrm{H}-5,17$ & 84.17 & 94.07 & 94.07 & 93.72 & 2.8414 & - & 22.63 & - \\
\hline $9 \mathrm{H}-5,32$ & 84.32 & 94.22 & 94.22 & 93.87 & 2.8482 & - & 21.95 & - \\
\hline $9 \mathrm{H}-5,47$ & 84.47 & 94.37 & 94.37 & 94.02 & 2.8550 & - & 21.95 & - \\
\hline $9 \mathrm{H}-5,62$ & 84.62 & 94.52 & 94.52 & 94.17 & 2.8619 & - & 21.95 & - \\
\hline $9 \mathrm{H}-5,77$ & 84.77 & 94.67 & 94.67 & 94.32 & 2.8687 & - & 21.95 & - \\
\hline $9 \mathrm{H}-5,92$ & 84.92 & 94.82 & 94.82 & 94,47 & 2.8755 & - & 21.95 & - \\
\hline
\end{tabular}


Table 1 (continued).

\begin{tabular}{|c|c|c|c|c|c|c|c|c|}
\hline \multirow[b]{2}{*}{$\begin{array}{l}\text { Core, section, } \\
\text { interval }(\mathrm{cm})\end{array}$} & \multirow[b]{2}{*}{$\begin{array}{c}\text { ODP } \\
\text { depth } \\
\text { (mbsf) }\end{array}$} & \multirow{2}{*}{$\begin{array}{c}\text { Shipboard } \\
\text { composite } \\
\text { depth } \\
\text { (mcd) }\end{array}$} & \multirow{2}{*}{$\begin{array}{l}\text { Revised } \\
\text { composite } \\
\text { depth } \\
\text { (rmcd) }\end{array}$} & \multirow{2}{*}{$\begin{array}{l}\text { Composite } \\
\text { depth, } \\
\text { this study } \\
\text { (Bmcd) }\end{array}$} & \multicolumn{2}{|c|}{ Ages } & \multicolumn{2}{|c|}{ Sedimentation rates } \\
\hline & & & & & $\begin{array}{l}\text { Shackleton } \\
\text { age model } \\
\text { (Ma) }\end{array}$ & $\begin{array}{c}\text { Isotope } \\
\text { age model } \\
\text { (Ma) }\end{array}$ & $\begin{array}{c}\text { Shackleton } \\
\text { ages } \\
(\mathrm{m} / \mathrm{m} . \mathrm{y} .)\end{array}$ & $\begin{array}{c}\text { Isotope } \\
\text { ages } \\
\text { (m/m.y.) }\end{array}$ \\
\hline $9 \mathrm{H}-5.107$ & 85.07 & 94,97 & 94.97 & 94.62 & 2.8828 & - & 20.79 & - \\
\hline $9 \mathrm{H}-5.122$ & 85.22 & 95.12 & 95.12 & 94.77 & 2.8900 & - & 20.71 & - \\
\hline $9 \mathrm{H}-5,137$ & 85.37 & 95.27 & 95.27 & 94.92 & 2.8972 & - & 20.71 & - \\
\hline $9 \mathrm{H}-6.2$ & 85.52 & 95.42 & 95.42 & 95.07 & 2.9042 & - & 21.44 & - \\
\hline $9 \mathrm{H}-6.17$ & 85.67 & 95.57 & 95.57 & 95.22 & 2.9078 & - & 41.82 & - \\
\hline $9 \mathrm{H}-6,32$ & 85.82 & 95.72 & 95.72 & 95.37 & 2.9114 & - & 41.82 & - \\
\hline $9 \mathrm{H}-6,47$ & 85.97 & 95.87 & 95.87 & 95.52 & 2.9150 & - & 41.82 & - \\
\hline $9 \mathrm{H}-6,62$ & 86.12 & 96.02 & 96.02 & 95.67 & 2.9186 & - & 41.82 & - \\
\hline $9 \mathrm{H}-6.77$ & 86.27 & 96.17 & 96.17 & 95.82 & 2.9222 & - & 41.82 & - \\
\hline $9 \mathrm{H}-6,92$ & 86.42 & 96.32 & 96.32 & 95.97 & 2.9258 & - & 41.82 & - \\
\hline $9 \mathrm{H}-6,107$ & 86.57 & 96.47 & 96.48 & 96.12 & 2.9283 & - & 59.91 & - \\
\hline $9 \mathrm{H}-6,122$ & 86.72 & 96.62 & 96.63 & 96.27 & 2.9307 & - & 61.82 & - \\
\hline $9 \mathrm{H}-6,137$ & 86.87 & 96.77 & 96.74 & 96.42 & 2.9331 & - & 61.82 & - \\
\hline $9 \mathrm{H}-7.2$ & 87.02 & 96.92 & 96.85 & 96.57 & 2.9355 & - & 61.82 & - \\
\hline $9 \mathrm{H}-7,17$ & 87.17 & 97.07 & 97.05 & 96.72 & 2.9407 & - & 29.14 & - \\
\hline $9 \mathrm{H}-7,32$ & 87.32 & 97.22 & 97.25 & 96.87 & 2.9496 & - & 16.87 & - \\
\hline $9 \mathrm{H}-7,48$ & 87.48 & 97.38 & 97.35 & 97.03 & 2.9558 & - & 25.75 & - \\
\hline $9 \mathrm{H}-7.62$ & 87.62 & 97.52 & 97.45 & 97.17 & 2.9612 & - & 25.75 & - \\
\hline $9 \mathrm{H}-7.77$ & 87.77 & 97.67 & 97.67 & 97.32 & 2.9671 & - & 25.75 & - \\
\hline
\end{tabular}

The section depth is the midpoint of a 4-cm sample.

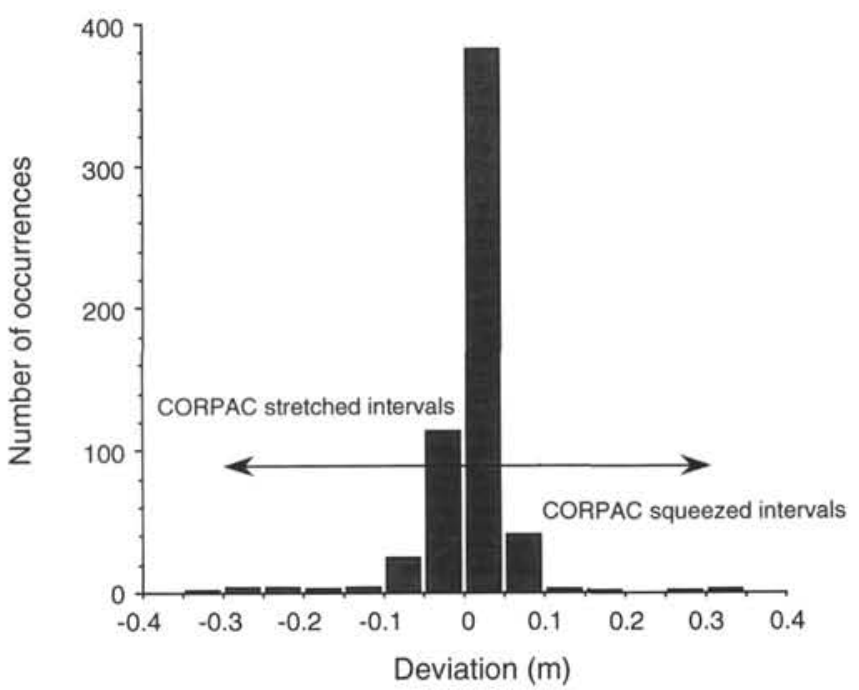

Figure 3. Histogram of the extent to which sample intervals have been stretched (negative values) or squeezed (positive values) when constructing revised composite depths (rmcd) for Hole 847C using the method of Hagelberg et al. (this volume). Of the 579 samples having a spacing near $15 \mathrm{~cm}$, 98 were stretched or squeezed by more than $5 \mathrm{~cm}$.

dried overnight at $100^{\circ} \mathrm{C}$ and then placed in a desiccator to cool for at least $1 \mathrm{hr}$.

Calcium carbonate content was measured on a gasometric apparatus similar to that described by Jones and Kaiteris (1983). The Brown University system uses a differential pressure gauge in place of a vacuum gauge, and carbonate reactions are measured at atmospheric pressure. The calcium carbonate was digested in $3 \mathrm{~mL}$ of $43 \%$ phosphoric acid (a 1:1 mixture of $85 \%$ phosphoric acid and deionized water). Replicate analyses of both standards and samples routinely give an analytical precision of better than $0.5 \%$ by weight. To ensure that our analyses are consistent with the shipboard data, we measured the calcite content on 22 samples from Site 847 that had been analyzed aboard the JOIDES Resolution using the coulometric procedure (Shipboard Scientific Party, 1992). No systematic offsets are apparent (Fig. 5), and the small differences are within the analytical errors of the two techniques.

Opal content was measured in the samples following the technique of Mortlock and Froelich (1989), except that buffered $0.5 \mathrm{M}$ acetic acid, rather than hydrochloric acid, was used to remove the carbonate material. A hot $\left(85^{\circ} \mathrm{C}\right) 2 \mathrm{M} \mathrm{Na}_{2} \mathrm{CO}_{3}$ leach was used to extract the biogenic opal. Samples were placed in a water bath for $6 \mathrm{hr}$ and stirred at 1- to 2-hr intervals. Measurements of dissolved silica were performed using a Spectronics 601 spectrophotometer; the precision based on standards and replicates was better than $1 \%$ by weight. Silicon concentrations were converted to opal using a factor of 2.5 , which applies to samples having a mixture of diatom and radiolarian skeletal material (Mortlock and Froelich, 1989). Residuals after the opal leach of selected samples were examined microscopically to ensure that complete or near-complete digestion of biogenic opal was obtained using a $2 \mathrm{M} \mathrm{Na}_{2} \mathrm{CO}_{3}$ extract solution. Some samples had relatively well-preserved radiolarian parts in the residuals. Additional experiments were performed to ensure that these undigested tests represented only a small portion of the total opal in the sample. Results of these experiments are reported in the Appendix. We conclude from these experiments that our procedure extracted most $(>95 \%)$ of the biogenic opal contained in these Site 847 samples.

\section{Dry Bulk Density}

Dry bulk density (DBD) data needed to calculate MARs were derived from the shipboard GRAPE wet-bulk-density (WBD) measurements. A total of 105 discrete DBD values were obtained by shipboard scientists within Cores $1 \mathrm{H}$ through $9 \mathrm{H}$ of Hole $847 \mathrm{~B}$ (Shipboard Scientific Party, 1992). These data are compared to the associated GRAPE values for each sample (Fig. 6). To obtain values for each sample depth, the GRAPE data, having a sample spacing of $\sim 2 \mathrm{~cm}$, were first smoothed with a nine-point Gaussian filter ( $T$. Hagelberg, pers. comm., 1993) and then sampled at the mod corresponding to the shipboard DBD measurements. This smoothing was necessary to reduce the high-frequency variability before interpolation to discrete sample depths. The linear regression between the GRAPE data and the discrete samples [DBD $=-1.695+1.538 *$ GRAPE; $r=0.94]$ was used to estimate the DBD for each sample in this study. The smoothed GRAPE records from Holes 847B, 847C, and $847 \mathrm{D}$ were sampled at the depths (mcd) corresponding to samples used in this study. These data are listed in Table 2.

\section{RESULTS AND DISCUSSION}

Site 847 is located west of the Galapagos Islands beneath highly productive surface waters associated with the equatorial divergence (Fig. 1). The sediments are largely a mixture of biogenic carbonate and opal, with lesser amounts of detrital material transported to the site by 


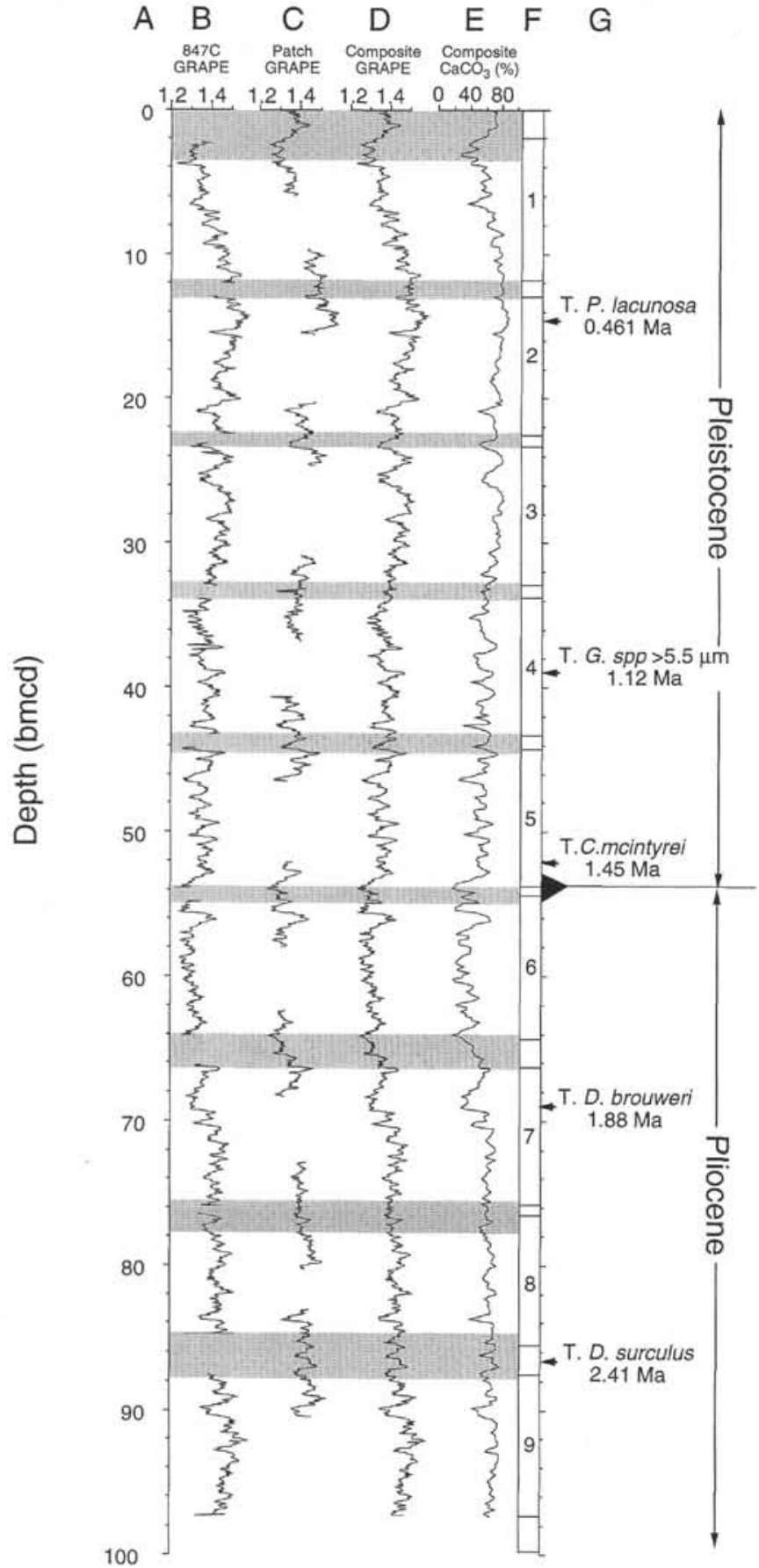

Figure 4. Composite depth reconstruction for the top nine cores in Site 847 using Hole $847 \mathrm{C}$ as the primary data and splicing in data from Holes $847 \mathrm{~B}$ and 847D to account for sediment missing or disturbed at core breaks (shaded areas). A. Depth below the seafloor in composite depth of this study (Bmcd). B. GRAPE data sampled at $\sim 2-\mathrm{cm}$ intervals from Hole $847 \mathrm{C}$ and smoothed using a nine-point Gaussian filter. C. GRAPE data from Holes 847B and 847D for the intervals spanning and adjacent to the core breaks in Hole $847 \mathrm{C}$. The first patch is from Hole 847B and all other patch data are from Hole 847D. D. Composite GRAPE data for Site 847 following the scheme listed in Table 3. E. $\% \mathrm{CaCO}_{3}$ data for the composite at Site 847 . Sample spacing is $\sim 15 \mathrm{~cm}$. Note that much of the structure in the GRAPE data is resolved in the more coarsely sampled carbonate data. F. Core numbers corresponding to Hole $847 \mathrm{C}$. G. Selected nannofossil datums used as control points for the shipboard sedimentation rate model for Site 847 (Shipboard Scientific Party, 1992). The location of the Pleistocene/Pliocene boundary was based on the nannofossil data reported for Site 847 (Shipboard Scientific Party, 1992).

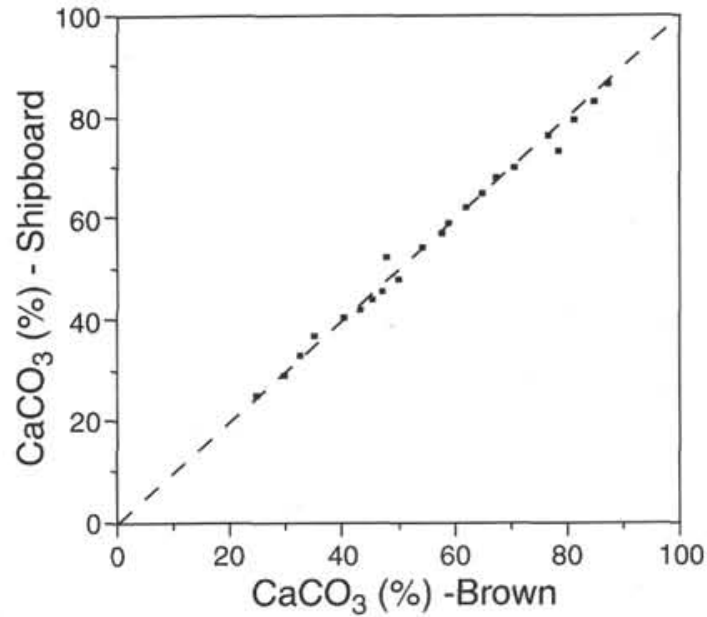

Figure 5. Scatter plot of calcium carbonate concentrations for 22 samples using the shipboard coulometric procedure (Shipboard Scientific Party, 1992) and gasometric technique. The 1:1 correspondence line is shown (dashed).

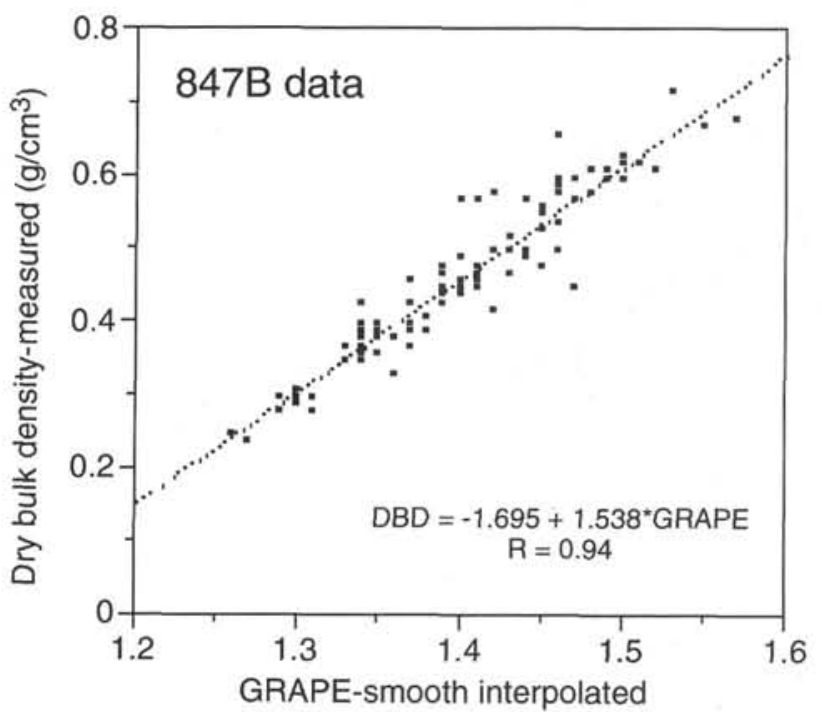

Figure 6. Scatter plot of discrete dry density values measured in Hole 847B samples in Cores $1 \mathrm{H}$ to $9 \mathrm{H}$ vs. the corresponding GRAPE density samples. The GRAPE data were smoothed using a nine-point Gaussian filter and were sampled at depths corresponding to the discrete dry-bulk-density (DBD) samples. The linear regression model based on these data and shown in the figure was used to calculate dry-bulk-density values for samples in this study.

means of winds (Fig. 7). At a water depth of $3334 \mathrm{~m}$, the site is near the top of the regional sediment lysocline (Berger et al., 1976), and the carbonate sediment component is relatively well preserved. Opal and carbonate concentrations have a high negative correlation (Fig. $8 \mathrm{~A}, r=-0.94)$; these two components mirror one another downcore (Fig. 7B). Because of the large contrast in the density and differential packing of carbonate and opal sediment components, the GRAPE WBD records exhibit a pattern that matches the relative changes in the proportions of carbonate and opal (Fig. 7A). A comparison of the opal and carbonate concentrations in the Site 847 samples to corresponding values of GRAPE density reveals a curvilinear relationship, with greater scatter in the high-opal, low-carbonate range (Figs. 8B$8 \mathrm{C})$. We tentatively attribute this larger scatter to the relative increase in a third component, detrital material, within some of the lowcarbonate intervals.

Mayer (1991) used the high correlation between GRAPE and calcium carbonate concentrations to derive an empirical relationship for 
Table 2. Data for Site 847 samples.

\begin{tabular}{|c|c|c|c|c|c|c|c|c|c|c|c|}
\hline $\begin{array}{l}\text { Core, section, } \\
\text { interval }(\mathrm{cm})\end{array}$ & $\begin{array}{c}\text { ODP } \\
\text { depth } \\
\text { (mbsf) }\end{array}$ & $\begin{array}{l}\text { Opal } \\
(\%)\end{array}$ & $\begin{array}{l}\text { Analyses } \\
\text { (\#) }\end{array}$ & $\mathrm{CaCO}_{3}$ & $\begin{array}{c}\text { Analyses } \\
\text { (\#) }\end{array}$ & $\begin{array}{c}\text { GRAPE } \\
\text { (smoothed and } \\
\text { interpolated) }\end{array}$ & $\begin{array}{c}\text { Estimated } \\
\mathrm{CaCO}_{3}\end{array}$ & $\begin{array}{l}\text { Dry bulk } \\
\text { density } \\
(\mathrm{g} / \mathrm{cm} 3)\end{array}$ & $\begin{array}{c}\text { Weight } \\
>150 \mu \mathrm{m} \\
(\%)\end{array}$ & $\begin{array}{c}\text { Whole } \\
\text { foraminifers } \\
(\%)\end{array}$ & $\begin{array}{c}\text { Radiolarians } \\
>150 \mu \mathrm{m} \\
(\%)\end{array}$ \\
\hline \multicolumn{12}{|l|}{ 138-847B- } \\
\hline IH-1, 2 & 0.02 & 19.37 & 1 & 66.05 & 2 & 1.340 & 46.44 & 0.366 & 5.46 & 54.75 & 37.35 \\
\hline $1 \mathrm{H}-\mathrm{I}, 19$ & 0.19 & 16.62 & 1 & 72.83 & 2 & 1.355 & 50.18 & 0.389 & 5,87 & 57.66 & 19.39 \\
\hline $1 \mathrm{H}-1,30$ & 0.30 & 18.76 & 1 & 70.12 & 2 & 1.392 & 58.78 & 0.446 & 10.52 & 76.22 & 18.59 \\
\hline IH-I, 47 & 0.47 & 15.46 & 1 & 71.41 & 2 & 1.393 & 58.99 & 0.447 & 12.53 & 74.52 & 16.56 \\
\hline IH-1, 59 & 0.59 & 15.95 & 1 & 69.60 & 2 & 1.367 & 53.05 & 0.407 & 9.31 & 57.65 & 25.00 \\
\hline $1 \mathrm{H}-1,77$ & 0.77 & 15.36 & 1 & 70.56 & 2 & 1.386 & 57.43 & 0.437 & 9.30 & 55.10 & 26.32 \\
\hline $1 \mathrm{H}-\mathrm{I}, 92$ & 0.92 & 17.57 & 1 & 70.79 & 2 & 1.379 & 55.84 & 0.426 & 9.99 & 48.08 & 29.91 \\
\hline $\mathrm{IH}-1,107$ & 1.07 & 18.05 & 1 & 73.19 & 2 & 1.437 & 67.99 & 0.515 & 10.32 & 68.31 & 20.33 \\
\hline IH-I, 123 & 1.23 & 18.40 & 1 & 69.98 & 2 & 1.404 & 61.34 & 0.464 & 7.27 & 58.84 & 27.77 \\
\hline $1 \mathrm{H}-1,137$ & 1.37 & 20.73 & 1 & 60.75 & 2 & 1.387 & 57.63 & 0.438 & 6.74 & 71.51 & 25.16 \\
\hline $1 \mathrm{H}-2.2$ & 1.52 & 26.30 & 1 & 61.06 & 1 & 1.365 & 52.55 & 0.404 & 5.88 & 66.89 & 26.92 \\
\hline $\mathrm{IH}-2,24$ & 1.74 & 28.14 & 1 & 61.58 & 1 & 1.353 & 49.63 & 0.386 & 9.79 & 80.58 & 10.34 \\
\hline $1 \mathrm{H}-2,37$ & 1.87 & 32.92 & 1 & 58.14 & 1 & 1.353 & 49.63 & 0.386 & 5.78 & 78.76 & 16.16 \\
\hline $1 \mathrm{H}-2,52$ & 2.02 & 33.22 & 1 & 50.30 & 1 & 1.321 & 41.41 & 0.337 & 8.40 & 79.57 & 20.59 \\
\hline IH-2, 67 & 2.17 & 31.45 & 1 & 44.33 & 1 & 1.317 & 40.32 & 0.331 & 2.66 & 70.29 & 36.79 \\
\hline $\mathrm{IH}-2,80$ & 2.30 & 32.70 & 1 & 42.07 & 1 & 1.273 & 27.79 & 0.263 & 2.73 & 72.63 & 47.34 \\
\hline $\mathrm{IH}-2,92$ & 2.42 & 33.66 & 1 & 37.62 & 1 & 1.246 & 19.45 & 0.221 & 2.41 & 52.15 & 69.06 \\
\hline IH-2, 107 & 2.57 & 32.97 & 1 & 43.99 & 1 & 1.277 & 28.97 & 0.269 & 4.56 & 60.23 & 51.14 \\
\hline $1 \mathrm{H}-2,123$ & 2.73 & 30.46 & 1 & 49.97 & 1 & 1.319 & 40.84 & 0.334 & 5.34 & 54.61 & 51.08 \\
\hline $1 \mathrm{H}-2,137$ & 2.87 & 31.61 & 1 & 41.79 & 1 & 1.303 & 36.45 & 0.309 & 5.10 & 71.82 & 46.99 \\
\hline $1 \mathrm{H}-3,2$ & 3.02 & 36.09 & 1 & 29.01 & 2 & 1.295 & 34.19 & 0.297 & 2.34 & 66.84 & 50.64 \\
\hline $1 \mathrm{H}-3,17$ & 3.17 & 37.42 & 1 & 28.14 & 2 & 1.278 & 29.24 & 0.271 & 1.97 & 51.78 & 73.12 \\
\hline $1 \mathrm{H}-3,30$ & 3.30 & 30.14 & 1 & 45.50 & 1 & 1.290 & 32.74 & 0.289 & 3.33 & 49.92 & 62.73 \\
\hline IH-3, 47 & 3.47 & 28.61 & 1 & 49.12 & 1 & 1.323 & 41.89 & 0.340 & 4.35 & 62.13 & 50.28 \\
\hline \multicolumn{12}{|l|}{$138-847 \mathrm{C}$} \\
\hline $1 \mathrm{H}-1,122$ & 3.22 & 31.16 & 1 & 48.57 & 1 & 1.319 & 40.81 & 0.334 & 6.66 & 69.13 & 57.00 \\
\hline $1 \mathrm{H}-1,137$ & 3.37 & 36.45 & 1 & 40.94 & 2 & 1.296 & 34.44 & 0.298 & 4.34 & 66.49 & 60.06 \\
\hline $\mathrm{IH}-2,2$ & 3.52 & 37.58 & 1 & 40.16 & 2 & 1.244 & 18.75 & 0.218 & 3.34 & 46.88 & 55.39 \\
\hline $1 \mathrm{H}-2,17$ & 3.67 & 26.32 & 1 & 59.77 & 1 & 1.351 & 49.06 & 0.383 & 4.85 & 55.52 & 60.45 \\
\hline IH-2, 32 & 3.82 & 27.79 & 1 & 56.83 & 1 & 1.356 & 50.29 & 0.391 & 7.25 & 52.21 & 67.82 \\
\hline IH-2, 47 & 3.97 & 27.68 & 1 & 53.44 & 1 & 1.348 & 48.31 & 0.378 & 6.59 & 67.84 & 47.51 \\
\hline IH-2, 65 & 4.15 & 23.61 & 1 & 59.86 & 1 & 1.354 & 49.79 & 0.387 & 5.89 & - & - \\
\hline $1 \mathrm{H}-2,77$ & 4.27 & 24.75 & 1 & 61.74 & 1 & 1.380 & 55.95 & 0.427 & 5.19 & 74.88 & 54.51 \\
\hline $1 \mathrm{H}-2,92$ & 4.42 & 22.45 & 1 & 65.52 & 1 & 1.376 & 55.02 & 0.421 & 5.17 & - & - \\
\hline IH-2, 107 & 4.57 & 24.71 & I & 59.02 & 1 & 1.359 & 50.99 & 0.395 & 4.75 & - & - \\
\hline $\mathrm{IH}-2,122$ & 4.72 & 31.80 & 1 & 52.23 & 1 & 1.333 & 44.46 & 0.355 & 5.43 & - & - \\
\hline IH-2, 137 & 4.87 & 29.93 & I & 54.48 & 1 & 1.348 & 48.27 & 0.378 & 3.64 & - & - \\
\hline $1 \mathrm{H}-3,2$ & 5.02 & 25.62 & 1 & 59.28 & 1 & 1.350 & 48.77 & 0.381 & 5.47 & - & - \\
\hline $1 \mathrm{H}-3,17$ & 5.17 & 27.13 & 1 & 56.87 & 1 & 1.340 & 46.24 & 0.366 & 2.99 & - & - \\
\hline $1 \mathrm{H}-3,32$ & 5.32 & 29.91 & I & 53.90 & 1 & 1.365 & 52.41 & 0.404 & 2.88 & - & - \\
\hline $1 \mathrm{H}-3,47$ & 5.47 & 20.00 & 1 & 65.86 & 1 & 1.377 & 55.22 & 0.423 & 2.99 & - & - \\
\hline IH-3, 62 & 5.62 & 21.48 & 1 & 64.74 & 1 & 1.374 & 54.52 & 0.418 & 3.50 & - & - \\
\hline IH-3, 77 & 5.77 & 25.18 & 1 & 55.30 & 1 & 1.347 & 47.99 & 0.377 & 3.34 & - & - \\
\hline $1 \mathrm{H}-3,92$ & 5.92 & 29.57 & 1 & 49.85 & 1 & 1.352 & 49.23 & 0.384 & 2.41 & - & - \\
\hline $1 \mathrm{H}-3,107$ & 6.07 & 29.52 & 1 & 48.56 & 1 & 1.318 & 40.44 & 0.332 & 1.87 & - & - \\
\hline $1 \mathrm{H}-3,122$ & 6.22 & 37.62 & 1 & 36.46 & 2 & 1.297 & 34.62 & 0.300 & 2.45 & - & - \\
\hline IH-3, 137 & 6.37 & 28.65 & 1 & 50.61 & 1 & 1.319 & 40.69 & 0.334 & 2.48 & - & - \\
\hline IH-4, 2 & 6.52 & 23.07 & 1 & 60.36 & 1 & 1.352 & 49.21 & 0.384 & 4.29 & - & - \\
\hline $1 \mathrm{H}-4,17$ & 6.67 & 25.30 & 1 & 59.75 & 1 & 1.338 & 45.68 & 0.363 & 2.80 & - & - \\
\hline $1 \mathrm{H}-4,32$ & 6.82 & 20.58 & 1 & 69.75 & 1 & 1.417 & 63.88 & 0.484 & 2.63 & - & - \\
\hline $\mathrm{IH}-4,47$ & 6.97 & 20.11 & 1 & 67.98 & 1 & 1.397 & 59.66 & 0.454 & 3.95 & - & - \\
\hline $1 \mathrm{H}-4,62$ & 7.12 & 20.03 & 1 & 66.51 & 1 & 1.379 & 55.63 & 0.426 & 3.34 & - & - \\
\hline $1 \mathrm{H}-4,77$ & 7.27 & 15.68 & 1 & 71.32 & 2 & 1.435 & 67.43 & 0.512 & 1.65 & - & - \\
\hline $\mathrm{IH}-4,92$ & 7.42 & 20.38 & I & 64.74 & 1 & 1.390 & 58.10 & 0.443 & 2.02 & - & - \\
\hline $\mathrm{IH}-4,107$ & 7.57 & 26.20 & 1 & 60.81 & 1 & 1.364 & 52.10 & 0.403 & 1.85 & - & - \\
\hline $1 \mathrm{H}-4,122$ & 7.72 & 27.70 & 1 & 60.02 & 2 & 1.357 & 50.40 & 0.392 & 2.77 & - & - \\
\hline $1 \mathrm{H}-4,137$ & 7.87 & 28.35 & 1 & 63.02 & 1 & 1.399 & 60.06 & 0.457 & 6.80 & - & - \\
\hline $1 \mathrm{H}-5,2$ & 8.02 & 22.01 & 1 & 71.66 & 1 & 1.422 & 64.86 & 0.492 & 3.64 & - & - \\
\hline IH-5, 17 & 8.17 & 10.46 & 1 & 75.93 & 1 & 1.434 & 67.21 & 0.511 & 4.29 & - & - \\
\hline IH-5. 32 & 8.32 & 9.51 & 1 & 81.62 & 2 & 1.477 & 74.89 & 0.577 & 5.07 & - & - \\
\hline IH-5, 47 & 8.47 & 13.30 & 1 & 74.77 & 1 & 1.428 & 66.04 & 0.501 & 5.61 & - & - \\
\hline IH-5. 62 & 8.62 & 16.56 & 1 & 68.24 & 1 & 1.402 & 60.69 & 0.461 & 4.34 & - & - \\
\hline $1 \mathrm{H}-5.77$ & 8.77 & 16.34 & 1 & 66.50 & I & 1.417 & 63.82 & 0.484 & 4.05 & - & - \\
\hline IH- 5,92 & 8.92 & 18.21 & 1 & 65.17 & 1 & 1.372 & 53.95 & 0.415 & 2.47 & - & - \\
\hline IH-5, 107 & 9.07 & 20.84 & 1 & 64.50 & 2 & 1.374 & $54.41^{\circ}$ & 0.418 & 2.12 & - & - \\
\hline IH-5, 122 & 9.22 & 14.35 & 1 & 76.75 & 1 & 1.467 & 73.20 & 0.561 & 2.47 & - & - \\
\hline IH-5, 137 & 9.37 & 12.64 & 1 & 75.80 & 1 & 1.433 & 66.99 & 0.509 & 3.10 & - & - \\
\hline $\mathrm{IH}-6,2$ & 9.52 & 11.89 & 1 & 75.89 & 1 & 1.477 & 74.87 & 0.577 & 2.53 & - & - \\
\hline $1 \mathrm{H}-6,17$ & 9.67 & 9.88 & 1 & 80.24 & 1 & 1.484 & 76.00 & 0.587 & 1.72 & - & - \\
\hline IH- 6,32 & 9.82 & 11.18 & 1 & 78.68 & 1 & 1.483 & 75.83 & 0.586 & 2.10 & - & - \\
\hline IH-6, 47 & 9.97 & 13.20 & 1 & 76.27 & 1 & 1.456 & 71.25 & 0.544 & 2.74 & - & - \\
\hline IH-6. 62 & 10.12 & 17.86 & 1 & 69.41 & 2 & 1.440 & 68.31 & 0.520 & 3.67 & - & - \\
\hline IH-6. 77 & 10.27 & 13.56 & 1 & 74.95 & 1 & 1.477 & 74.85 & 0.577 & 2.79 & - & - \\
\hline IH- 6,92 & 10.42 & 12.73 & 1 & 78.43 & 2 & 1.486 & 76.30 & 0.591 & 4.48 & - & - \\
\hline 1H-6. 107 & 10.57 & 13.78 & 1 & 74.44 & 1 & 1.445 & 69.24 & 0.527 & 9.93 & - & - \\
\hline IH- 6,122 & 10.72 & 16.54 & 1 & 73.00 & 1 & 1.436 & 67.53 & 0.514 & 9.35 & - & - \\
\hline $1 \mathrm{H}-6,137$ & 10.87 & 13.22 & 1 & 74.43 & 1 & 1.478 & 75.00 & 0.578 & 4.27 & - & - \\
\hline $1 \mathrm{H}-7,2$ & 11.02 & 8.68 & 1 & 81.50 & 1 & 1.493 & 77.38 & 0.601 & 2.09 & - & - \\
\hline $1 \mathrm{H}-7,17$ & 11.17 & 8.34 & 1 & 82.26 & 1 & 1.549 & 84.96 & 0.687 & 3.37 & - & - \\
\hline IH-7, 32 & 11.32 & 9.32 & 1 & 81.12 & 1 & 1.527 & 82.23 & 0.654 & 2.45 & - & - \\
\hline
\end{tabular}


Table 2 (continued).

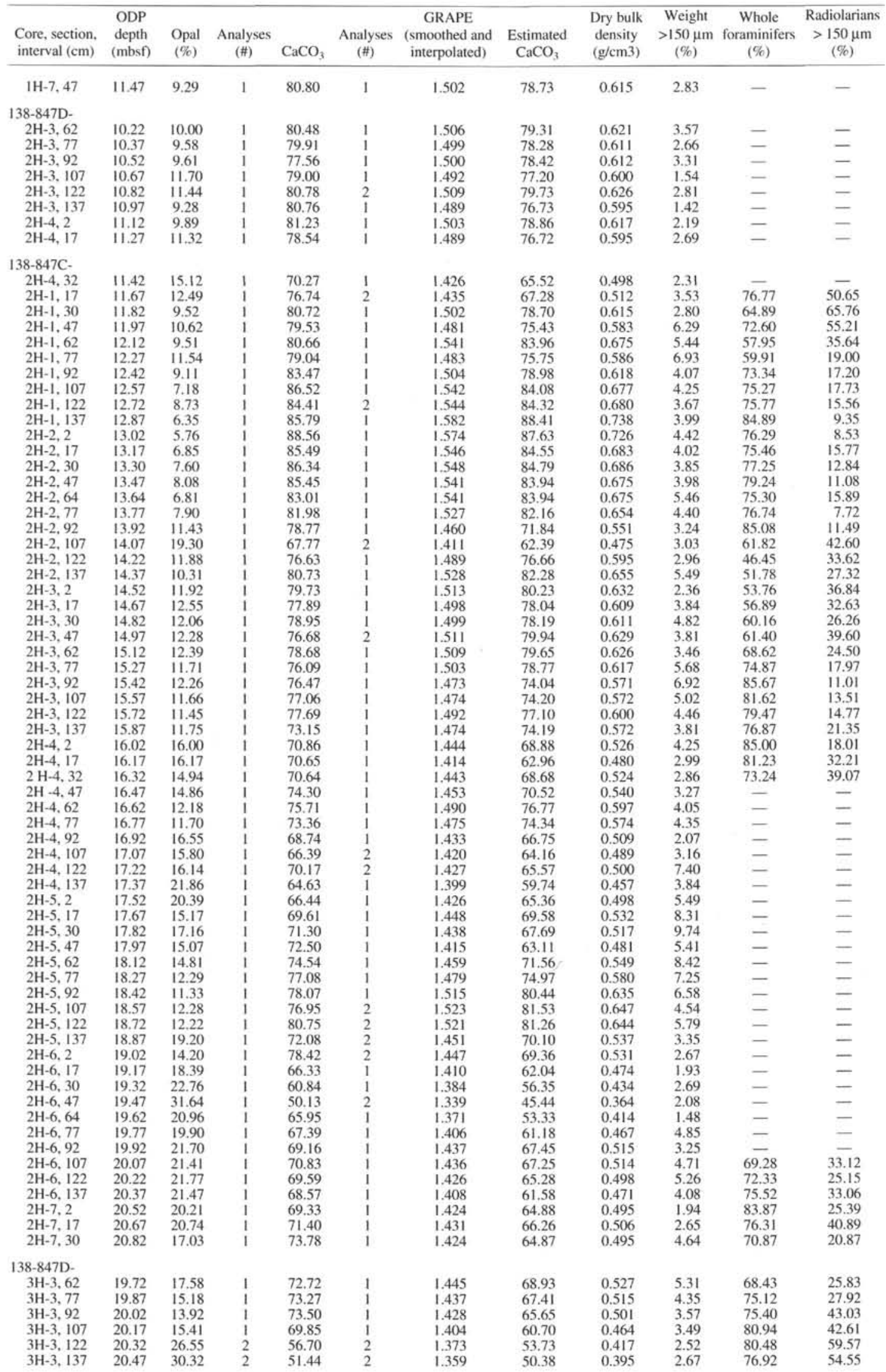


Table 2 (continued).

\begin{tabular}{|c|c|c|c|c|c|c|c|c|c|c|c|}
\hline $\begin{array}{l}\text { Core, section. } \\
\text { interval }(\mathrm{cm})\end{array}$ & $\begin{array}{l}\text { ODP } \\
\text { depth } \\
\text { (mbsf) }\end{array}$ & $\begin{array}{l}\text { Opal } \\
(\%)\end{array}$ & $\begin{array}{c}\text { Analyses } \\
\text { (\#) }\end{array}$ & $\mathrm{CaCO}_{3}$ & $\begin{array}{c}\text { Analyses } \\
\text { (\#) }\end{array}$ & $\begin{array}{c}\text { GRAPE } \\
\text { (smoothed and } \\
\text { interpolated) }\end{array}$ & $\begin{array}{c}\text { Estimated } \\
\mathrm{CaCO}_{3}\end{array}$ & $\begin{array}{c}\text { Dry bulk } \\
\text { density } \\
(\mathrm{g} / \mathrm{cm} 3)\end{array}$ & $\begin{array}{l}\text { Weight } \\
>150 \mu \mathrm{m} \mathrm{f} \\
(\%)\end{array}$ & $\begin{array}{c}\text { Whole } \\
\text { foraminifers } \\
(\%)\end{array}$ & $\begin{array}{c}\text { Radiolarians } \\
>150 \mu \mathrm{m} \\
(\%)\end{array}$ \\
\hline \multicolumn{12}{|l|}{ 138-847C- } \\
\hline $3 \mathrm{H}-1,17$ & 21.17 & 26.11 & 1 & 56.42 & 2 & 1.385 & 56.50 & 0.435 & 2.87 & 78.38 & 64.16 \\
\hline $3 \mathrm{H}-1,32$ & 21.32 & 22.53 & 1 & 61.02 & 1 & 1.395 & 58.73 & 0.451 & 3.69 & 72.67 & 68.07 \\
\hline $3 \mathrm{H}-1.47$ & 21.47 & 15.24 & 1 & 73.38 & 1 & 1.412 & 62.37 & 0.477 & 2.36 & 42.66 & 73.69 \\
\hline $3 \mathrm{H}-1,62$ & 21.62 & 15.52 & 1 & 74.47 & 1 & 1.435 & 67.00 & 0.512 & 2.94 & 54.50 & 37.21 \\
\hline $3 \mathrm{H}-1,77$ & 21.77 & 13.60 & 2 & 78.90 & i & 1.457 & 71.09 & 0.546 & 2.68 & 68.72 & 23.77 \\
\hline $3 \mathrm{H}-1,92$ & 21.92 & 11.26 & 2 & 79.44 & i & 1.498 & 77.87 & 0.609 & 4.28 & 64.22 & 29.58 \\
\hline $3 \mathrm{H}-1,107$ & 22.07 & 12.74 & 2 & 79.78 & 1 & 1.469 & 73.18 & 0.564 & 4.71 & 67.07 & 30.04 \\
\hline $3 \mathrm{H}-1,122$ & 22.22 & 9.42 & 1 & 81.13 & 1 & 1.491 & 76.78 & 0.598 & 6.47 & 68.49 & 19.54 \\
\hline $3 \mathrm{H}-1.137$ & 22.37 & 12.63 & 1 & 79.92 & 1 & 1.458 & 71.26 & 0.547 & 6.96 & 65.22 & 14.86 \\
\hline $3 \mathrm{H}-2,2$ & 22.52 & 19.04 & i & 72.93 & i & 1.450 & 69.80 & 0.535 & 5.37 & 63.81 & 38.26 \\
\hline $3 \mathrm{H}-2,17$ & 22.67 & 21.70 & i & 71.37 & i & 1.422 & 64.39 & 0.492 & 5.16 & 66.79 & 29.98 \\
\hline $3 \mathrm{H}-2,30$ & 22.82 & 20.31 & I & 68.73 & I & 1.416 & 63.16 & 0.483 & 5.18 & 58.18 & 40.41 \\
\hline $3 \mathrm{H}-2,47$ & 22.97 & 25.74 & i & 61.46 & i & 1.380 & 55.30 & 0.427 & 5.20 & - & - \\
\hline $3 \mathrm{H}-2,65$ & 23.12 & 27.69 & 1 & 57.83 & 1 & 1.353 & 48.83 & 0.386 & 6.93 & 49.73 & 65.41 \\
\hline $3 \mathrm{H}-2,77$ & 23.27 & 28.54 & 2 & 59.34 & i & 1.386 & 56.66 & 0.437 & 5.39 & - & - \\
\hline $3 \mathrm{H}-2,92$ & 23.42 & 26.62 & 2 & 54.33 & 2 & 1.345 & 46.81 & 0.374 & 8.34 & - & - \\
\hline $3 \mathrm{H}-2,107$ & 23.57 & 28.60 & 2 & 55.96 & 1 & 1.367 & 52.23 & 0.407 & 5.16 & 44.22 & 75.22 \\
\hline $3 \mathrm{H}-2,122$ & 23.72 & 26.12 & 1 & 62.79 & 1 & 1.387 & 56.87 & 0.438 & 5.69 & - & - \\
\hline $3 \mathrm{H}-2.137$ & 23.87 & 25.69 & 1 & 63.55 & 1 & 1.399 & 59.53 & 0.457 & 7.62 & - & - \\
\hline $3 \mathrm{H}-3,2$ & 24.02 & 14.42 & i & 74.87 & i & 1.451 & 69.95 & 0.537 & 11.82 & - & - \\
\hline $3 \mathrm{H}-3,17$ & 24.17 & 15.95 & 1 & 74.25 & 1 & 1.432 & 66.34 & 0.507 & 6.68 & - & - \\
\hline $3 \mathrm{H}-3,32$ & 24.32 & 12.31 & 1 & 78.45 & 1 & 1.447 & 69.20 & 0.531 & 5.23 & - & - \\
\hline $3 \mathrm{H}-3,47$ & 24.47 & 12.88 & 1 & 78.23 & 1 & 1.498 & 77.82 & 0.609 & 6.26 & - & - \\
\hline $3 \mathrm{H}-3,62$ & 24.62 & 10.46 & I & 81.37 & 1 & 1.486 & 75.94 & 0.591 & 5.61 & - & - \\
\hline $3 \mathrm{H}-3,76$ & 24.76 & 10.75 & I & 79.81 & 1 & 1.495 & 77.35 & 0.604 & 5.23 & - & - \\
\hline $3 \mathrm{H}-3,92$ & 24.92 & 10.90 & 1 & 81.74 & 1 & 1.492 & 76.88 & 0.600 & 5.14 & - & - \\
\hline $3 \mathrm{H}-3,107$ & 25.07 & 16.20 & 1 & 78.03 & 1 & 1.484 & 75.61 & 0.587 & 7.80 & - & - \\
\hline $3 \mathrm{H}-3,122$ & 25.22 & 15.82 & i & 76.33 & i & 1.472 & 73.62 & 0.569 & 6.19 & - & - \\
\hline $3 \mathrm{H}-3,137$ & 25.37 & 20.20 & 3 & 74.32 & i & 1.490 & 76.56 & 0.597 & 5.08 & - & - \\
\hline $3 \mathrm{H}-4,2$ & 25.52 & 18.41 & 3 & 74.16 & 1 & 1.482 & 75.27 & 0.584 & 4.13 & - & - \\
\hline $3 \mathrm{H}-4.17$ & 25.67 & 11.39 & 1 & 79.08 & i & 1.490 & 76.55 & 0.597 & 4.83 & - & - \\
\hline $3 \mathrm{H}-4,32$ & 25.82 & 12.11 & 1 & 78.98 & 1 & 1.492 & 76.86 & 0.600 & 4.86 & - & - \\
\hline $3 \mathrm{H}-4,47$ & 25.97 & 19.35 & i & 68.58 & i & 1.431 & 66.10 & 0.506 & 3.87 & - & - \\
\hline $3 \mathrm{H}-4,62$ & 26.12 & 19.91 & i & 68.43 & i & 1.411 & 62.03 & 0.475 & 3.28 & - & - \\
\hline $3 \mathrm{H}-4,76$ & 26.26 & 17.00 & I & 68.41 & 1 & 1.387 & 56.79 & 0.438 & 4.86 & - & - \\
\hline $3 \mathrm{H}-4,92$ & 26.42 & 16.62 & 1 & 72.99 & 1 & 1.448 & 69.33 & 0.532 & 2.09 & - & - \\
\hline $3 \mathrm{H}-4,107$ & 26.57 & 15.89 & i & 74.89 & i & 1.441 & 68.02 & 0.521 & 2.97 & - & - \\
\hline $3 \mathrm{H}-4,122$ & 26.72 & 15.64 & i & 73.50 & i & 1.459 & 71.33 & 0.549 & 3.89 & - & - \\
\hline $3 \mathrm{H}-4,137$ & 26.87 & 11.91 & i & 77.02 & i & 1.475 & 74.09 & 0.574 & 3.76 & - & - \\
\hline $3 \mathrm{H}-5,2$ & 27.02 & 13.01 & I & 76.34 & i & 1.494 & 77.15 & 0.603 & 6.83 & - & - \\
\hline $3 \mathrm{H}-5,17$ & 27.17 & 18.35 & 1 & 71.98 & 2 & 1.440 & 67.81 & 0.520 & 3.14 & - & - \\
\hline $3 \mathrm{H}-5,32$ & 27.32 & 13.44 & i & 79.22 & 2 & 1.464 & 72.19 & 0.557 & 6.21 & - & - \\
\hline $3 \mathrm{H}-5,47$ & 27.47 & 13.47 & 1 & 75.30 & 1 & 1.429 & 65.66 & 0.503 & 8.66 & - & - \\
\hline $3 \mathrm{H}-5,62$ & 27.62 & 15.58 & 1 & 73.43 & I & 1.428 & 65.46 & 0.501 & 4.28 & - & - \\
\hline $3 \mathrm{H}-5,76$ & 27.76 & 18.55 & 1 & 74.17 & 1 & 1.455 & 70.58 & 0.543 & 5.44 & - & - \\
\hline $3 \mathrm{H}-5,92$ & 27.92 & 17.70 & i & 76.83 & i & 1.467 & 72.70 & 0.561 & 5.37 & - & - \\
\hline $3 \mathrm{H}-5,107$ & 28.07 & 13.18 & i & 78.09 & i & 1.485 & 75.70 & 0.589 & 8.08 & - & - \\
\hline $3 \mathrm{H}-5,122$ & 28.22 & 18.26 & i & 74.68 & i & 1.448 & 69.29 & 0.532 & 5.69 & - & - \\
\hline $3 \mathrm{H}-5,137$ & 28.37 & 21.22 & I & 67.66 & i & 1.414 & 62.59 & 0.480 & 3.90 & - & - \\
\hline $3 \mathrm{H}-6.2$ & 28.52 & 25.39 & 1 & 63.16 & 2 & 1.420 & 63.82 & 0.489 & 4.05 & - & - \\
\hline $3 \mathrm{H}-6,17$ & 28.67 & 22.30 & 1 & 67.67 & 1 & 1.406 & 60.89 & 0.467 & 4.12 & - & - \\
\hline $3 \mathrm{H}-6,30$ & 28.82 & 19.18 & I & 72.50 & I & 1.430 & 65.82 & 0.504 & 7.43 & - & - \\
\hline $3 \mathrm{H}-6,47$ & 28.97 & 18.93 & 1 & 71.62 & I & 1.445 & 68.71 & 0.527 & 6.79 & - & - \\
\hline $3 \mathrm{H}-6,65$ & 29.12 & 16.17 & 1 & 75.81 & 1 & 1.476 & 74.20 & 0.575 & 5.38 & - & - \\
\hline $3 \mathrm{H}-6,77$ & 29.27 & 18.11 & i & 73.45 & i & 1.484 & 75.52 & 0.587 & 5.80 & - & - \\
\hline $3 \mathrm{H}-6,92$ & 29.42 & 23.77 & i & 68.03 & i & 1.424 & 64.61 & 0.495 & 5.29 & - & - \\
\hline $3 \mathrm{H}-6,107$ & 29.57 & 27.99 & 2 & 63.08 & i & 1.417 & 63.18 & 0.484 & 4.01 & - & - \\
\hline $3 \mathrm{H}-6,122$ & 29.72 & 24.88 & 1 & 64.08 & i & 1.403 & 60.22 & 0.463 & 6.01 & - & - \\
\hline $3 \mathrm{H}-6,137$ & 29.87 & 30.43 & i & 57.44 & i & 1.380 & 55.08 & 0.427 & 3.23 & - & - \\
\hline $3 \mathrm{H}-7.2$ & 30.02 & 36.73 & 2 & 53.55 & 2 & 1.399 & 59.34 & 0.457 & 3.36 & - & - \\
\hline $3 \mathrm{H}-7,17$ & 30.17 & 22.38 & 1 & 67.81 & 1 & 1.404 & 60.42 & 0.464 & 6.25 & - & - \\
\hline $3 \mathrm{H}-7,30$ & 30.30 & 15.93 & 1 & 73.19 & 2 & 1.425 & 64.79 & 0.497 & 6.87 & - & - \\
\hline $3 \mathrm{H}-7,47$ & 30.47 & 23.07 & 1 & 67.56 & 1 & 1.395 & 58.45 & 0.451 & 8.46 & - & - \\
\hline $3 \mathrm{H}-7,62$ & 30.62 & 27.03 & i & 61.62 & $i$ & 1.396 & 58.67 & 0.452 & 6.07 & - & - \\
\hline \multicolumn{12}{|l|}{ 138-847D- } \\
\hline $4 \mathrm{H}-3,47$ & 29.07 & 28.10 & 3 & 60.12 & 1 & 1.403 & 60.19 & 0.463 & - & - & - \\
\hline $4 \mathrm{H}-3,62$ & 29.22 & 29.87 & 3 & 58.62 & I & 1.404 & 60.40 & 0.464 & - & - & - \\
\hline $4 \mathrm{H}-3,77$ & 29.37 & 25.56 & 3 & 64.98 & i & 1.415 & 62.72 & 0.481 & - & - & - \\
\hline $4 \mathrm{H}-3,92$ & 29.52 & 22.66 & 3 & 53.06 & 1 & 1.370 & 52.70 & 0.412 & - & - & - \\
\hline $4 \mathrm{H}-3,107$ & 29.67 & 24.63 & 3 & 57.93 & i & 1.389 & 57.09 & 0.441 & - & - & - \\
\hline $4 \mathrm{H}-3,122$ & 29.82 & 28.81 & 3 & 58.77 & i & 1.403 & 60.17 & 0.463 & - & - & - \\
\hline $4 \mathrm{H}-3,137$ & 29.97 & 29.01 & 3 & 59.55 & 1 & 1.408 & 61.24 & 0.471 & - & - & - \\
\hline $4 \mathrm{H}-1,32$ & 30.82 & 28.00 & 1 & 62.93 & 1 & 1.393 & 57.97 & 0.447 & 5.03 & - & - \\
\hline $4 \mathrm{H}-1,47$ & 30.97 & 27.24 & I & 62.83 & 1 & 1.369 & 52.43 & 0.411 & 6.46 & - & - \\
\hline $4 \mathrm{H}-1,61$ & 31.11 & 30.99 & 2 & 58.87 & i & 1.348 & 47.28 & 0.378 & 5.26 & - & - \\
\hline $4 \mathrm{H}-1,77$ & 31.27 & 26.62 & 1 & 54.22 & 2 & 1.341 & 45.49 & 0.367 & 5.30 & - & - \\
\hline $4 \mathrm{H}-1,92$ & 31,42 & 23.61 & 1 & 58.98 & 1 & 1.372 & 53.13 & 0.415 & 4.63 & - & - \\
\hline $4 \mathrm{H}-1,107$ & 31.57 & 24.28 & 1 & 63.53 & 2 & 1.381 & 55.23 & 0.429 & 5.36 & - & - \\
\hline $4 \mathrm{H}-1,122$ & 31.72 & 29.14 & i & 57.09 & 1 & 1.365 & 51.45 & 0.404 & 4.74 & - & - \\
\hline $4 \mathrm{H}-1.137$ & 31.87 & 37.01 & 3 & 45.74 & 2 & 1.339 & 44.95 & 0.364 & 3.68 & - & - \\
\hline $\begin{array}{r}38-847 \mathrm{C}- \\
4 \mathrm{H}-2,2\end{array}$ & 32.02 & 31.44 & 3 & 39.59 & 1 & 1.293 & 32.36 & 0.294 & - & - & - \\
\hline
\end{tabular}


Table 2 (continued).

\begin{tabular}{|c|c|c|c|c|c|c|c|c|c|c|c|}
\hline $\begin{array}{l}\text { Core, section, } \\
\text { interval }(\mathrm{cm})\end{array}$ & $\begin{array}{c}\text { ODP } \\
\text { depth } \\
\text { (mbsf) }\end{array}$ & $\begin{array}{l}\text { Opal } \\
(\%)\end{array}$ & $\begin{array}{c}\text { Analyses } \\
\text { (\#) }\end{array}$ & $\mathrm{CaCO}_{3}$ & $\begin{array}{c}\text { Analyses } \\
\text { (\#) }\end{array}$ & $\begin{array}{c}\text { GRAPE } \\
\text { (smoothed and } \\
\text { interpolated) }\end{array}$ & $\begin{array}{c}\text { Estimated } \\
\mathrm{CaCO}_{3}\end{array}$ & $\begin{array}{c}\text { Dry bulk } \\
\text { density } \\
(\mathrm{g} / \mathrm{cm} 3)\end{array}$ & $\begin{array}{c}\text { Weight } \\
>150 \mu \mathrm{m} \\
(\%)\end{array}$ & $\begin{array}{c}\text { Whole } \\
\text { foraminifers } \\
(\%)\end{array}$ & $\begin{array}{c}\text { Radiolarians } \\
>150 \mu \mathrm{m} \\
(\%)\end{array}$ \\
\hline $4 \mathrm{H}-2,17$ & 32.17 & 32.62 & 1 & 49.86 & 1 & 1.331 & 42.85 & 0.352 & - & - & - \\
\hline $4 \mathrm{H}-2,32$ & 32.32 & 31.05 & 1 & 53.79 & 1 & 1.330 & 42.58 & 0.351 & - & - & - \\
\hline $4 \mathrm{H}-2,47$ & 32.47 & 30.48 & $i$ & 52.30 & i & 1.312 & 37.71 & 0.323 & - & - & - \\
\hline $4 \mathrm{H}-2,63.5$ & 32.64 & 27.49 & $i$ & 53.61 & i & 1.357 & 49.47 & 0.392 & - & - & - \\
\hline $4 \mathrm{H}-2,77$ & 32.77 & 26.42 & 1 & 56.24 & 1 & 1.311 & 37.42 & 0.321 & - & - & - \\
\hline $4 \mathrm{H}-2,88$ & 32.88 & 26.61 & 1 & 52.84 & 1 & 1.336 & 44.14 & 0.360 & - & - & - \\
\hline $4 \mathrm{H}-2,107$ & 33.07 & 26.74 & 1 & 58.05 & 1 & 1.383 & 55.64 & 0.432 & - & - & - \\
\hline $4 \mathrm{H}-2,122$ & 33.22 & 28.49 & 1 & 56.50 & i & I. 348 & 47.20 & 0.378 & - & - & - \\
\hline $4 \mathrm{H}-2,137$ & 33.37 & 29.01 & I & 59.22 & 1 & 1.408 & 61.16 & 0.471 & - & - & - \\
\hline $4 \mathrm{H}-3,2$ & 33.52 & 26.20 & 1 & 62.68 & I & 1.371 & 52.83 & 0.414 & - & - & - \\
\hline $4 \mathrm{H}-3,17$ & 33.67 & 18.03 & 1 & 67.80 & I & 1.401 & 59.64 & 0.460 & - & - & - \\
\hline $4 \mathrm{H}-3,32$ & 33.82 & 13.17 & 1 & 72.90 & I & 1.420 & 63.65 & 0.489 & - & - & - \\
\hline $4 \mathrm{H}-3,47$ & 33.97 & 14.73 & i & 72.90 & i & 1.440 & 67.61 & 0.520 & - & - . & - \\
\hline $4 \mathrm{H}-3,61$ & 34.11 & 13.14 & 1 & 72.48 & i & 1.453 & 70.04 & 0.540 & - & - & - \\
\hline $4 \mathrm{H}-3,77$ & 34.27 & 15.75 & 1 & 67.50 & 1 & i. 333 & 43.30 & 0.355 & - & - & - \\
\hline $4 \mathrm{H}-3,92$ & 34.42 & 22.48 & i & 58.83 & i & 1.354 & 48.67 & 0.387 & - & - & - \\
\hline $4 \mathrm{H}-3,107$ & 34.57 & 31.91 & $i$ & 45.12 & i & I. 343 & 45.89 & 0.371 & - & - & - \\
\hline $4 \mathrm{H}-3,122$ & 34.72 & 32.84 & I & 46.37 & 1 & 1.304 & 35.39 & 0.311 & - & - & - \\
\hline $4 \mathrm{H}-3,137$ & 34.87 & 32.58 & 1 & 51.13 & 1 & 1.351 & 47.90 & 0.383 & - & - & - \\
\hline $4 \mathrm{H}-4,2$ & 35.02 & 31.43 & 1 & 48.92 & i & 1.353 & 48.40 & 0.386 & - & - & - \\
\hline $4 \mathrm{H}-4,17$ & 35.17 & 36.17 & i & 48.30 & i & 1.338 & 44.58 & 0.363 & - & - & - \\
\hline $4 \mathrm{H}-4,30$ & 35.30 & 35.40 & 1 & 51.93 & 1 & 1.355 & 48.89 & 0.389 & - & - & - \\
\hline $4 \mathrm{H}-4,47$ & 35.47 & 32.25 & 1 & 50.12 & 1 & 1.362 & 50.60 & 0.400 & - & - & - \\
\hline $4 \mathrm{H}-4,6 \mathrm{I}$ & 35.61 & 27.81 & 1 & 57.09 & 1 & 1.369 & 52.28 & 0.411 & - & - & - \\
\hline $4 \mathrm{H}-4,77$ & 35.77 & 16.89 & $i$ & 69.28 & i & 1.424 & 64.41 & 0.495 & - & - & - \\
\hline $4 \mathrm{H}-4,92$ & 35.92 & 15.64 & 1 & 68.51 & 1 & 1.472 & 73.35 & 0.569 & - & - & - \\
\hline $4 \mathrm{H}-4,107$ & 36.07 & 17.69 & 1 & 67.00 & i & 1.426 & 64.80 & 0.498 & - & - & - \\
\hline $4 \mathrm{H}-4,122$ & 36.22 & 23.47 & I & 58.19 & I & 1.396 & 58.47 & 0.452 & - & - & - \\
\hline $4 \mathrm{H}-4,137$ & 36.37 & 24.67 & 1 & 56.82 & 1 & 1.390 & 57.13 & 0.443 & - & - & - \\
\hline $4 \mathrm{H}-5,2$ & 36.52 & 35.98 & 2 & 41.84 & i & 1.352 & 48.10 & 0.384 & - & - & - \\
\hline $4 \mathrm{H}-5,17$ & 36.67 & 26.03 & 1 & 57.82 & 1 & 1.367 & 51.77 & 0.407 & - & - & - \\
\hline $4 \mathrm{H}-5,30$ & 36.80 & 27.06 & 1 & 59.24 & 1 & 1.408 & 61.06 & 0.471 & - & - & - \\
\hline $4 \mathrm{H}-5,47$ & 36.97 & 22.48 & 1 & 67.93 & 1 & 1.412 & 61.90 & 0.477 & - & - & - \\
\hline $4 \mathrm{H}-5,61$ & 37.11 & 18.40 & 1 & 69.79 & 1 & 1.442 & 67.91 & 0.523 & - & - & - \\
\hline $4 \mathrm{H}-5,77$ & 37.27 & 15.87 & 1 & 70.65 & 1 & 1.442 & 67.90 & 0.523 & - & - & - \\
\hline $4 \mathrm{H}-5,92$ & 37.42 & 19.98 & I & 68.08 & 1 & 1.426 & 64.77 & 0.498 & - & - & - \\
\hline $4 \mathrm{H}-5,107$ & 37.57 & 20.70 & 3 & 64.03 & 1 & 1.406 & 60.61 & 0.467 & - & - & - \\
\hline $4 \mathrm{H}-5,122$ & 37.72 & 27.68 & 1 & 56.08 & 1 & 1.384 & 55.72 & 0.434 & - & - & - \\
\hline $4 \mathrm{H}-5,137$ & 37.87 & 31.77 & 1 & 50.52 & 1 & 1.382 & 55.26 & 0.431 & - & - & - \\
\hline $4 \mathrm{H}-6,2$ & 38.02 & 35.51 & 1 & 48.51 & 1 & 1.365 & 51.24 & 0.404 & - & - & - \\
\hline $4 \mathrm{H}-6,17$ & 38.17 & 30.94 & 1 & 57.03 & i & 1.390 & 57.07 & 0.443 & - & - & - \\
\hline $4 \mathrm{H}-6,29$ & 38.29 & 31,14 & 1 & 56.80 & i & 1.375 & 53.62 & 0.420 & - & - & - \\
\hline $4 \mathrm{H}-6,47$ & 38.47 & 30.75 & 3 & 54.58 & 1 & 1.367 & 51.71 & 0.407 & - & - & - \\
\hline $4 \mathrm{H}-6,64$ & 38.64 & 34.12 & 2 & 53.20 & 1 & 1.380 & 54.77 & 0.427 & - & - & - \\
\hline $4 \mathrm{H}-6,77$ & 38.77 & 23.23 & 2 & 63.61 & 1 & 1.393 & 57.73 & 0.447 & - & - & - \\
\hline $4 \mathrm{H}-6,92$ & 38.92 & 34.08 & 2 & 45.85 & i & 1.397 & 58.61 & 0.454 & - & - & - \\
\hline $4 \mathrm{H}-6,107$ & 39.07 & 22.86 & 1 & 59.44 & 1 & 1.418 & 63.09 & 0.486 & - & - & - \\
\hline $4 \mathrm{H}-6,122$ & 39.22 & 17.80 & 1 & 65.38 & 1 & 1.378 & 54.29 & 0.424 & - & - & - \\
\hline $4 \mathrm{H}-6,137$ & 39.37 & 19.87 & 1 & 61.31 & 1 & 1.313 & 37.73 & 0.324 & - & - & - \\
\hline $4 \mathrm{H}-7,2$ & 39.52 & 44.41 & 2 & 32.20 & 1 & 1.320 & 39.64 & 0.335 & - & - & - \\
\hline $4 \mathrm{H}-7.17$ & 39.67 & 32.77 & 1 & 50.72 & 1 & 1.324 & 40.72 & 0.341 & - & - & - \\
\hline $4 \mathrm{H}-7.32$ & 39.82 & 21.91 & 1 & 59.22 & 1 & 1.412 & 61.82 & 0.477 & - & - & - \\
\hline $4 \mathrm{H}-7,47$ & 39.97 & 21.14 & 1 & 66.13 & 1 & 1.430 & 65.49 & 0.504 & - & - & - \\
\hline \multicolumn{12}{|l|}{ 138-847D- } \\
\hline $5 \mathrm{H}-3.107$ & 39.17 & 18.69 & 3 & 66.20 & 1 & 1.400 & 59.23 & 0.458 & - & - & - \\
\hline $5 \mathrm{H}-3,122$ & 39.32 & 21.00 & 3 & 64.49 & 1 & 1.388 & 56.56 & 0.440 & - & - & - \\
\hline $5 \mathrm{H}-3,137$ & 39.47 & 23.83 & 3 & 63.94 & 1 & 1.402 & 59.66 & 0.461 & - & - & - \\
\hline $5 \mathrm{H}-4,2$ & 39.62 & 25.86 & 3 & 62.68 & 1 & 1.417 & 62.84 & 0.484 & - & - & - \\
\hline $5 \mathrm{H}-4.17$ & 39.77 & 22.75 & 3 & 60.95 & 1 & 1.375 & 53.54 & 0.420 & - & - & - \\
\hline $5 \mathrm{H}-4,32$ & 39.92 & 24.41 & 1 & 58.49 & 1 & 1.348 & 46.94 & 0.378 & - & - & - \\
\hline $5 \mathrm{H}-4.47$ & 40.07 & 35.95 & 1 & 43.72 & 2 & 1.321 & 39.86 & 0.337 & - & - & - \\
\hline $5 \mathrm{H}-4.62$ & 40.22 & 26.39 & 1 & 57.52 & 1 & 1.341 & 45.14 & 0.367 & - & - & - \\
\hline $5 \mathrm{H}-4,77$ & 40.37 & 21.64 & 1 & 66.40 & 1 & 1.410 & 61.35 & 0.474 & - & - & - \\
\hline $5 \mathrm{H}-4,92$ & 40.52 & 16.94 & 1 & 74.55 & 2 & 1.489 & 76.03 & 0.595 & - & - & - \\
\hline $138-847 \mathrm{C}$ & & & & & & & & & & & \\
\hline $5 \mathrm{H}-1.47$ & 40.47 & 10.55 & 1 & 74.20 & 1 & 1.461 & 71.29 & 0.552 & - & - & - \\
\hline $5 \mathrm{H}-1.62$ & 40.62 & 19.24 & 1 & 67.18 & 1 & 1.407 & 60.70 & 0.469 & - & - & - \\
\hline $5 \mathrm{H}-1.77$ & 40.77 & 29.17 & 1 & 50.80 & 1 & 1.368 & 51.84 & 0.409 & - & - & - \\
\hline $5 \mathrm{H}-1,92$ & 40.92 & 25.85 & 1 & 51.42 & 1 & 1.344 & 45.88 & 0.372 & - & - & - \\
\hline $5 \mathrm{H}-1,107$ & 41.07 & 21.87 & 1 & 64.09 & 1 & 1.409 & 61.11 & 0.472 & - & - & - \\
\hline $5 \mathrm{H}-\mathrm{I}, 122$ & 41.22 & 19.59 & 2 & 64.68 & 1 & 1.412 & 61.74 & 0.477 & - & - & - \\
\hline $5 \mathrm{H}-1,137$ & 41.37 & 26.75 & 1 & 58.11 & 1 & 1.400 & 59.16 & 0.458 & - & - & - \\
\hline $5 \mathrm{H}-2,2$ & 41.52 & 27.83 & 1 & 57.10 & 1 & 1.393 & 57.61 & 0.447 & - & - & - \\
\hline $5 \mathrm{H}-2,17$ & 41.67 & 21.17 & 1 & 62.59 & 1 & 1.398 & 58.71 & 0.455 & - & - & - \\
\hline $5 \mathrm{H}-2,28$ & 41.78 & 23.93 & 1 & 54.14 & 1 & 1.360 & 49.86 & 0.397 & - & - & - \\
\hline $5 \mathrm{H}-2,47$ & 41.97 & 31.23 & 1 & 43.55 & i & 1.318 & 38.96 & 0.332 & - & - & - \\
\hline $5 \mathrm{H}-2,64$ & 42.14 & 46.69 & 1 & 30.34 & 1 & 1.295 & 32.49 & 0.297 & - & - & - \\
\hline $5 \mathrm{H}-2,77$ & 42.27 & 49.40 & 1 & 26.97 & 1 & 1.285 & 29.57 & 0.281 & - & - & - \\
\hline $5 \mathrm{H}-2,92$ & 42.42 & 34.81 & 1 & 44.45 & 1 & 1.291 & 31.32 & 0.291 & - & - & - \\
\hline $5 \mathrm{H}-2.107$ & 42.57 & 19.56 & 3 & 57.79 & 1 & 1.348 & 46.84 & 0.378 & - & - & - \\
\hline $5 \mathrm{H}-2,122$ & 42.72 & 23.59 & 1 & 56.58 & 1 & 1.348 & 46.84 & 0.378 & - & - & - \\
\hline $5 \mathrm{H}-2,137$ & 42.87 & 23.52 & i & 59.05 & 1 & 1.387 & 56.21 & 0.438 & - & - & - \\
\hline $5 \mathrm{H}-3.2$ & 43.02 & 19.61 & i & 62.49 & 2 & 1.381 & 54.83 & 0.429 & - & - & - \\
\hline
\end{tabular}


Table 2 (continued).

\begin{tabular}{|c|c|c|c|c|c|c|c|c|c|c|c|}
\hline $\begin{array}{l}\text { Core, section, } \\
\text { interval }(\mathrm{cm})\end{array}$ & $\begin{array}{c}\text { ODP } \\
\text { depth } \\
\text { (mbsf) }\end{array}$ & $\begin{array}{l}\text { Opal } \\
(\%)\end{array}$ & $\begin{array}{c}\text { Analyses } \\
\text { (\#) }\end{array}$ & $\mathrm{CaCO}_{3}$ & $\begin{array}{c}\text { Analyses } \\
\text { (\#) }\end{array}$ & $\begin{array}{c}\text { GRAPE } \\
\text { (smoothed and } \\
\text { interpolated) }\end{array}$ & $\begin{array}{c}\text { Estimated } \\
\mathrm{CaCO}_{3}\end{array}$ & $\begin{array}{c}\text { Dry bulk } \\
\text { density } \\
(\mathrm{g} / \mathrm{cm} 3)\end{array}$ & $\begin{array}{l}\text { Weight } \\
>150 \mu \mathrm{m} \\
(\%)\end{array}$ & $\begin{array}{l}\text { Whole } \\
\text { foraminifers } \\
(\%)\end{array}$ & $\begin{array}{c}\text { Radiolarians } \\
>150 \mu \mathrm{m} \\
(\%)\end{array}$ \\
\hline $5 \mathrm{H}-3,17$ & 43.17 & 20.28 & 1 & 60.86 & 1 & 1.373 & 52.95 & 0.417 & - & - & - \\
\hline $5 \mathrm{H}-3,28$ & 43.28 & 22.64 & i & 59.74 & i & 1.362 & 50.30 & 0.400 & - & - & - \\
\hline $5 \mathrm{H}-3,47$ & 43.47 & 30.50 & 1 & 47.50 & 1 & 1.308 & 36.14 & 0.317 & - & - & - \\
\hline $5 \mathrm{H}-3,62$ & 43.62 & 26.86 & 1 & 53.19 & I & 1.325 & 40.80 & 0.343 & - & - & - \\
\hline $5 \mathrm{H}-3,77$ & 43.77 & 26.32 & 1 & 52.62 & 1 & 1.351 & 47.56 & 0.383 & - & - & - \\
\hline $5 \mathrm{H}-3,92$ & 43,92 & 22.54 & i & 59.86 & i & 1.372 & 52.69 & 0.415 & - & - & - \\
\hline $5 \mathrm{H}-3,107$ & 44.07 & 19.96 & i & 61.34 & i & 1.403 & 59.73 & 0.463 & - & - & - \\
\hline $5 \mathrm{H}-3,122$ & 44.22 & 21.61 & i & 59.83 & i & 1.371 & 52.44 & 0.414 & - & - & - \\
\hline $5 \mathrm{H}-3,137$ & 44.37 & 27.73 & 1 & 46.34 & 1 & 1.333 & 42.90 & 0.355 & - & - & - \\
\hline $5 \mathrm{H}-4.2$ & 44.52 & 30.56 & 1 & 45.93 & 1 & 1.338 & 44.21 & 0.363 & - & - & - \\
\hline $5 \mathrm{H}-4,17$ & 44.67 & 26.76 & 1 & 47.69 & i & 1.297 & 32.97 & 0.300 & - & - & - \\
\hline $5 \mathrm{H}-4,28$ & 44.78 & 22.61 & 1 & 51.04 & 1 & 1.331 & 42.36 & 0.352 & - & - & - \\
\hline $5 \mathrm{H}-4,47$ & 44.97 & 25.57 & 1 & 47.38 & 1 & 1.329 & 41.82 & 0.349 & - & - & - \\
\hline $5 \mathrm{H}-4,62$ & 45.12 & 16.99 & 1 & 61.84 & 1 & 1.388 & 56.37 & 0.440 & - & - & - \\
\hline $5 \mathrm{H}-4,77$ & 45.27 & 14.61 & 2 & 70.19 & i & 1.438 & 66,89 & 0.517 & - & - & - \\
\hline $5 \mathrm{H}-4,92$ & 45.42 & 18.35 & $i$ & 62.06 & i & 1.409 & 60.98 & 0.472 & - & - & - \\
\hline $5 \mathrm{H}-4,107$ & 45.57 & 26.49 & i & 51.03 & i & 1.374 & 53.11 & 0.418 & - & - & - \\
\hline $5 \mathrm{H}-4,122$ & 45.72 & 24.99 & I & 51.05 & 1 & 1.358 & 49.24 & 0.394 & - & - & - \\
\hline $5 \mathrm{H}-4,137$ & 45.87 & 23.37 & i & 50.15 & 2 & 1.336 & 43.64 & 0.360 & - & - & - \\
\hline $5 \mathrm{H}-5,2$ & 46.02 & 31.28 & i & 33.07 & 1 & 1.337 & 43.89 & 0.361 & - & - & - \\
\hline $5 \mathrm{H}-5,17$ & 46.17 & 28.34 & I & 45.06 & 1 & 1.295 & 32.34 & 0.297 & - & - & - \\
\hline $5 \mathrm{H}-5.28$ & 46.28 & 18.68 & 1 & 63.63 & I & 1.404 & 59.88 & 0.464 & - & - & - \\
\hline $5 \mathrm{H}-5,47$ & 46.47 & 18.82 & i & 65.49 & 1 & 1.436 & 66.47 & 0.514 & - & - & - \\
\hline $5 \mathrm{H}-5,62$ & 46.62 & 20.77 & i & 58.62 & i & 1.399 & 58.78 & 0.457 & - & - & - \\
\hline $5 \mathrm{H}-5,77$ & 46.77 & 24.20 & i & 51.77 & i & 1.352 & 47.71 & 0.384 & - & - & - \\
\hline $5 \mathrm{H}-5,92$ & 46.92 & 19.44 & 1 & 56.88 & 1 & 1.372 & 52.59 & 0.415 & - & - & - \\
\hline $5 \mathrm{H}-5,107$ & 47.07 & 21.09 & 1 & 53.93 & 1 & 1.356 & 48.70 & 0.391 & - & - & - \\
\hline $5 \mathrm{H}-5,122$ & 47.22 & 19.16 & i & 61.86 & I & 1.409 & 60.93 & 0.472 & - & - & - \\
\hline $5 \mathrm{H}-5,137$ & 47.37 & 18.34 & i & 63.62 & i & 1.381 & 54.69 & 0.429 & - & - & - \\
\hline $5 \mathrm{H}-6,2$ & 47.52 & 24.12 & i & 55.32 & i & 1.387 & 56.07 & 0.438 & - & - & - \\
\hline $5 \mathrm{H}-6.17$ & 47.67 & 28.43 & 1 & 49.40 & I & 1.339 & 44.35 & 0.364 & - & - & - \\
\hline $5 \mathrm{H}-6,28$ & 47.78 & 27.52 & 1 & 49.12 & 1 & 1.344 & 45.64 & 0.372 & - & - & - \\
\hline $5 \mathrm{H}-6.47$ & 47.97 & 23.41 & 1 & 52.70 & I & 1.380 & 54.44 & 0.427 & - & - & - \\
\hline $5 \mathrm{H}-6.64$ & 48.14 & 23.36 & i & 50.40 & i & 1.351 & 47.41 & 0.383 & - & - & - \\
\hline $5 \mathrm{H}-6,77$ & 48.27 & 23.27 & 2 & 53.50 & I & 1.340 & 44.59 & 0.366 & - & - & - \\
\hline $5 \mathrm{H}-6,92$ & 48.42 & 19.18 & 1 & 57.62 & I & 1.361 & 49.89 & 0.398 & - & - & - \\
\hline $5 \mathrm{H}-6,107$ & 48.57 & 17.50 & i & 65.34 & i & 1.375 & 53.25 & 0.420 & - & - & - \\
\hline $5 \mathrm{H}-6,122$ & 48.72 & 18.14 & i & 61.32 & i & 1.419 & 62.99 & 0.487 & - & - & - \\
\hline $5 \mathrm{H}-6,137$ & 48.87 & 22.93 & i & 56.26 & i & 1.365 & 50.85 & 0.404 & - & - & - \\
\hline $5 \mathrm{H}-7.2$ & 49.02 & 28.95 & 1 & 46,47 & i & 1.364 & 50.60 & 0.403 & - & - & - \\
\hline $5 \mathrm{H}-7,17$ & 49.17 & 31.47 & 1 & 41.28 & 1 & 1.311 & 36.76 & 0.321 & - & - & - \\
\hline $5 \mathrm{H}-7,28$ & 49.28 & 34.66 & i & 37.09 & $i$ & 1.320 & 39.24 & 0.335 & - & - & - \\
\hline $5 \mathrm{H}-7,47$ & 49.47 & 38.06 & 1 & 23.91 & i & 1.299 & 33.36 & 0.303 & - & - & - \\
\hline $5 \mathrm{H}-7,64$ & 49.64 & 39.65 & 1 & 22.54 & 1 & 1.261 & 21.99 & 0.244 & - & - & - \\
\hline \multicolumn{12}{|l|}{ 138-847D- } \\
\hline $6 \mathrm{H}-3,32$ & 47.92 & 36.99 & 2 & 19.28 & 2 & 1.285 & 29.28 & 0.281 & - & - & - \\
\hline $6 \mathrm{H}-3.47$ & 48.07 & 32.01 & 1 & 25.54 & 2 & 1.277 & 26.88 & 0.269 & - & - & - \\
\hline $6 \mathrm{H}-3,62$ & 48.22 & 27.53 & i & 47.83 & I & 1.309 & 36.18 & 0.318 & - & - & - \\
\hline $6 \mathrm{H}-3,77$ & 48.37 & 31.78 & 1 & 39.92 & I & 1.310 & 36.45 & 0.320 & - & - & - \\
\hline $6 \mathrm{H}-3,92$ & 48.52 & 37.43 & i & 30.12 & i & 1.292 & 31.31 & 0.292 & - & - & - \\
\hline $6 \mathrm{H}-3,107$ & 48.67 & 32.81 & 1 & 36.76 & I & 1.302 & 34.18 & 0.308 & - & - & - \\
\hline $6 \mathrm{H}-3,122$ & 48.82 & 26.34 & i & 50.04 & i & 1.349 & 46.82 & 0.380 & - & - & - \\
\hline $6 \mathrm{H}-3,137$ & 48.97 & 26.36 & 1 & 52.26 & 1 & 1.345 & 45.80 & 0.374 & - & - & - \\
\hline $138-847 \mathrm{C}$ - & & & & & & & & & & & \\
\hline $6 \mathrm{H}-1,17$ & 49.67 & 35.42 & 1 & 31.38 & 1 & 1.287 & 29.82 & 0.284 & 1.92 & 79.48 & - \\
\hline $6 \mathrm{H}-1,30$ & 49.80 & 39.42 & 1 & 30.83 & 1 & 1.283 & 28.63 & 0.278 & 1.97 & 81.53 & - \\
\hline $6 \mathrm{H}-1,47$ & 49.97 & 40.88 & 1 & 22.63 & 1 & 1.262 & 22.23 & 0.246 & 2.52 & 82.94 & - \\
\hline $6 \mathrm{H}-1,62$ & 50.12 & 35.43 & i & 22.75 & 2 & 1.278 & 27.12 & 0.271 & 1.71 & 59.69 & - \\
\hline $6 \mathrm{H}-1,77$ & 50.27 & 23.63 & i & 45.53 & 1 & 1.249 & 18.12 & 0.226 & 4.18 & 41.16 & - \\
\hline $6 \mathrm{H}-1,92$ & 50.42 & 20.43 & i & 60,70 & i & 1.375 & 53.15 & 0.420 & 4.86 & 44.25 & - \\
\hline $6 \mathrm{H}-1,107$ & 50.57 & 19.32 & 1 & 64.29 & 1 & 1.409 & 60.80 & 0.472 & 3.31 & 39.94 & - \\
\hline $6 \mathrm{H}-1,122$ & 50.72 & 15.35 & 1 & 66.03 & 1 & 1.381 & 54.55 & 0.429 & 6.37 & 39.11 & - \\
\hline $6 \mathrm{H}-1,137$ & 50.87 & 17.63 & i & 66.93 & 1 & 1.414 & 61.85 & 0.480 & 5.58 & 53.20 & - \\
\hline $6 \mathrm{H}-2,2$ & 51.02 & 26.01 & i & 55.85 & i & 1.383 & 55.00 & 0.432 & 6.13 & 66.07 & - \\
\hline $6 \mathrm{H}-2,17$ & 51.17 & 32.23 & 1 & 44.37 & i & 1.355 & 48.27 & 0.389 & 4.21 & 65.38 & - \\
\hline $6 \mathrm{H}-2,30$ & 51.30 & 32.20 & i & 44.63 & 1 & 1.333 & 42.61 & 0.355 & 3.12 & 68.73 & - \\
\hline $6 \mathrm{H}-2,47$ & 51.47 & 36.08 & i & 38.06 & i & 1.302 & 34.09 & 0.308 & 2.74 & 65.37 & - \\
\hline $6 \mathrm{H}-2,58$ & 51.58 & 39.11 & I & 31.95 & 1 & 1.310 & 36.35 & 0.320 & 2.61 & 55.05 & - \\
\hline $6 \mathrm{H}-2,77$ & 51.77 & 41.22 & i & 24.51 & i & 1.266 & 23.40 & 0.252 & 2.57 & 66.67 & - \\
\hline $6 \mathrm{H}-2,92$ & 51.92 & 30.83 & 1 & 39.57 & 1 & 1.310 & 36.34 & 0.320 & 2.29 & 36.54 & - \\
\hline $6 \mathrm{H}-2,107$ & 52.07 & 31.32 & 1 & 40.46 & 1 & 1.317 & 38.27 & 0.331 & 4.12 & 31.65 & - \\
\hline $6 \mathrm{H}-2,122$ & 52.22 & 38.08 & $\mathrm{i}$ & 27.66 & i & 1.269 & 24.30 & 0.257 & 5.37 & 66.94 & - \\
\hline $6 \mathrm{H}-2,137$ & 52.37 & 38.78 & i & 28.55 & i & 1.293 & 31.47 & 0.294 & 2.54 & 46.05 & - \\
\hline $6 \mathrm{H}-3,2$ & 52.52 & 39.92 & 1 & 27.43 & 1 & 1.289 & 30.29 & 0.288 & 7.01 & 47.93 & - \\
\hline $6 \mathrm{H}-3,17$ & 52.67 & 35.81 & i & 38.01 & i & 1.285 & 29.11 & 0.281 & 4.54 & 56.65 & - \\
\hline $6 \mathrm{H}-3,29$ & 52.79 & 32.07 & i & 43.71 & i & 1.311 & 36.58 & 0.321 & 4.00 & 66.75 & - \\
\hline $6 \mathrm{H}-3,47$ & 52.97 & 36.25 & i & 41.76 & i & 1.327 & 40.95 & 0.346 & 6.85 & 60.61 & - \\
\hline $6 \mathrm{H}-3,62$ & 53.12 & 32.81 & i & 41.51 & 1 & 1.323 & 39.87 & 0.340 & 5.00 & 55.54 & - \\
\hline $6 \mathrm{H}-3,77$ & 53.27 & 42.08 & i & 34.11 & i & 1.306 & 35.16 & 0.314 & 5.20 & 47.57 & - \\
\hline $6 \mathrm{H}-3,92$ & 53.42 & 42.56 & i & 29.50 & i & 1.261 & 21.78 & 0.244 & 4.67 & - & - \\
\hline $6 \mathrm{H}-3,107$ & 53.57 & 40.13 & 3 & 26.85 & 1 & 1.271 & 24.86 & 0.260 & 2.72 & - & - \\
\hline $6 \mathrm{H}-3,122$ & 53.72 & 45.85 & i & 23.02 & 1 & 1.264 & 22.70 & 0.249 & 2.39 & - & - \\
\hline & & 45.94 & 1 & 24.82 & 1 & 1.261 & 21.76 & 0.244 & 3.96 & 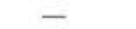 & \\
\hline
\end{tabular}


Table 2 (continued).

\begin{tabular}{|c|c|c|c|c|c|c|c|c|c|c|c|}
\hline $\begin{array}{l}\text { Core, section, } \\
\text { interval }(\mathrm{cm})\end{array}$ & $\begin{array}{l}\text { ODP } \\
\text { depth } \\
\text { (mbsf) }\end{array}$ & $\begin{array}{c}\text { Opal } \\
(\%)\end{array}$ & $\begin{array}{l}\text { Analyses } \\
\text { (\#) }\end{array}$ & $\mathrm{CaCO}_{3}$ & $\begin{array}{c}\text { Analyses } \\
\text { (\#) }\end{array}$ & $\begin{array}{c}\text { GRAPE } \\
\text { (smoothed and } \\
\text { interpolated) }\end{array}$ & $\begin{array}{c}\text { Estimated } \\
\mathrm{CaCO}_{3}\end{array}$ & $\begin{array}{l}\text { Dry bulk } \\
\text { density } \\
(\mathrm{g} / \mathrm{cm} 3)\end{array}$ & $\begin{array}{c}\text { Weight } \\
>150 \mu \mathrm{m} \\
(\%)\end{array}$ & $\begin{array}{c}\text { Whole } \\
\text { foraminifers } \\
(\%)\end{array}$ & $\begin{array}{c}\text { Radiolarians } \\
>150 \mu \mathrm{m} \\
(\%)\end{array}$ \\
\hline $6 \mathrm{H}-4,2$ & 54.02 & 44.88 & 1 & 33.81 & 1 & 1.321 & 39.29 & 0.337 & 3.57 & 56.54 & - \\
\hline $6 \mathrm{H}-4,17$ & 54.17 & 49.37 & $i$ & 27.96 & i & 1.300 & 33.42 & 0.304 & 3.63 & 71.79 & - \\
\hline $6 \mathrm{H}-4,28$ & 54.28 & 48.38 & 1 & 25.48 & 1 & 1.282 & 28.15 & 0.277 & 2.08 & 39.88 & - \\
\hline $6 \mathrm{H}-4,47$ & 54.47 & 35.87 & 2 & 41.33 & 1 & 1.318 & 38.46 & 0.332 & 4.52 & 64.44 & - \\
\hline $6 \mathrm{H}-4,62$ & 54.62 & 46.38 & 1 & 32.28 & 1 & 1.295 & 31.96 & 0.297 & 3.18 & - & - \\
\hline $6 \mathrm{H}-4,77$ & 54.77 & 44.60 & 1 & 32.21 & 2 & 1.311 & 36.51 & 0.321 & 2.97 & - & - \\
\hline $6 \mathrm{H}-4,92$ & 54.92 & 48.89 & $i$ & 19.23 & $\overrightarrow{1}$ & 1.296 & 32.24 & 0.298 & 2.59 & - & - \\
\hline $6 \mathrm{H}-4,107$ & 55.07 & 50.45 & 1 & 22.70 & 1 & 1.285 & 29.01 & 0.281 & 1.71 & - & - \\
\hline $6 \mathrm{H}-4,122$ & 55.22 & 46.46 & 1 & 34.74 & 1 & 1.311 & 36.49 & 0.321 & 3.20 & - & - \\
\hline $6 \mathrm{H}-4,137$ & 55.37 & 35.01 & 1 & 45.78 & I & 1.308 & 35.64 & 0.317 & 3.84 & - & - \\
\hline $6 \mathrm{H}-5,2$ & 55.52 & 28.71 & 1 & 44.56 & 1 & 1.339 & 44.04 & 0.364 & 3.82 & - & - \\
\hline $6 \mathrm{H}-5,17$ & 55.67 & 33.83 & 1 & 41.16 & 1 & 1.313 & 37.03 & 0.324 & 3.18 & - & - \\
\hline $6 \mathrm{H}-5,28$ & 55.78 & 39.24 & i & 36.95 & $i$ & 1.311 & 36.47 & 0.321 & 4.09 & - & - \\
\hline $6 \mathrm{H}-5,47$ & 55.97 & 33.48 & 1 & 43.66 & 1 & 1.331 & 41.91 & 0.352 & 2.64 & - & - \\
\hline $6 \mathrm{H}-5,62$ & 56.12 & 29.16 & 1 & 49.85 & 1 & 1.323 & 39.76 & 0.340 & 3.52 & - & - \\
\hline $6 \mathrm{H}-5,77$ & 56.27 & 25.79 & i & 52.34 & $i$ & 1.352 & 47.34 & 0.384 & 3.63 & - & - \\
\hline $6 \mathrm{H}-5,92$ & 56.42 & 31.00 & i & 43.45 & i & 1.347 & 46.07 & 0.377 & 3.07 & - & - \\
\hline $6 \mathrm{H}-5,107$ & 56.57 & 41.42 & 1 & 32.19 & 1 & 1.310 & 36.16 & 0.320 & 2.96 & - & - \\
\hline $6 \mathrm{H}-5,122$ & 56.72 & 47.87 & 1 & 23.66 & 1 & 1.290 & 30.42 & 0.289 & 2.04 & - & - \\
\hline $6 \mathrm{H}-5,137$ & 56.87 & 38.54 & 1 & 37.83 & 1 & 1.322 & 39.46 & 0.338 & 2.37 & - & - \\
\hline $6 \mathrm{H}-6,2$ & 57.02 & 37.72 & 1 & 37.50 & 1 & 1.317 & 38.09 & 0.331 & 2.22 & - & - \\
\hline $6 \mathrm{H}-6,17$ & 57.17 & 36.30 & 1 & 30.17 & 2 & 1.304 & 34.45 & 0.311 & 2.76 & - & - \\
\hline $6 \mathrm{H}-6,28$ & 57.28 & 35.18 & 1 & 37.55 & 1 & 1.346 & 45.78 & 0.375 & 2.14 & - & - \\
\hline $6 \mathrm{H}-6,47$ & 57.47 & 36.62 & 1 & 36.09 & 1 & 1.316 & 37.79 & 0.329 & 2.16 & - & - \\
\hline $6 \mathrm{H}-6,58$ & 57.58 & 27.30 & 1 & 45.08 & 1 & 1.332 & 42.12 & 0.354 & 2.51 & - & - \\
\hline $6 \mathrm{H}-6,77$ & 57.77 & 33.31 & 1 & 43.92 & 1 & 1.348 & 46.28 & 0.378 & 2.99 & - & - \\
\hline $6 \mathrm{H}-6,92$ & 57.92 & 23.20 & 1 & 55.05 & 1 & 1.369 & 51.48 & 0.411 & 3.18 & - & - \\
\hline $6 \mathrm{H}-6,107$ & 58.07 & 29.55 & 1 & 48.06 & 1 & 1.349 & 46.52 & 0.380 & 3.12 & - & - \\
\hline $6 \mathrm{H}-6,122$ & 58.22 & 31.11 & 1 & 46.77 & 1 & 1.343 & 44.98 & 0.371 & 1.88 & - & - \\
\hline $6 \mathrm{H}-6,137$ & 58.37 & 41.29 & 1 & 36.18 & 1 & 1.342 & 44.71 & 0.369 & 1.90 & - & - \\
\hline $6 \mathrm{H}-7,2$ & 58.52 & 36.92 & 1 & 30.60 & 1 & 1.316 & 37.75 & 0.329 & 1.80 & - & - \\
\hline $6 \mathrm{H}-7,17$ & 58.67 & 47.41 & 2 & 27.11 & 1 & 1.310 & 36.08 & 0.320 & 1.69 & - & - \\
\hline \multicolumn{12}{|l|}{ 138-847D- } \\
\hline $7 \mathrm{H}-3,17$ & 57.27 & 50.42 & 1 & 19.59 & 2 & 1.276 & 26.17 & 0.268 & - & - & - \\
\hline $7 \mathrm{H}-3,32$ & 57.42 & 44.53 & $i$ & 30.20 & I & 1.278 & 26.77 & 0.271 & - & - & - \\
\hline $7 \mathrm{H}-3,47$ & 57.57 & 41.19 & 1 & 33.92 & 1 & 1.291 & 30.62 & 0.291 & - & - & - \\
\hline $7 \mathrm{H}-3,62$ & 57.72 & 38.93 & 1 & 36.92 & 1 & 1.297 & 32.36 & 0.300 & - & - & - \\
\hline $7 \mathrm{H}-3,77$ & 57.87 & 39.53 & 1 & 40.00 & 1 & 1.316 & 37.72 & 0.329 & - & - & - \\
\hline $7 \mathrm{H}-3,92$ & 58.02 & 34.90 & 1 & 45.41 & 1 & 1.315 & 37.44 & 0.328 & - & - & - \\
\hline $7 \mathrm{H}-3,107$ & 58.17 & 33.81 & 2 & 47.28 & I & 1.330 & 41.51 & 0.351 & - & - & - \\
\hline $7 \mathrm{H}-3,122$ & 58.32 & 36.82 & 2 & 44.40 & 1 & 1.318 & 38.26 & 0.332 & - & - & - \\
\hline $7 \mathrm{H}-3,137$ & 58.47 & 34.87 & I & 45.76 & 1 & 1.313 & 36.87 & 0.324 & - & - & - \\
\hline $7 \mathrm{H}-4,2$ & 58.62 & 31.87 & I & 48.09 & 1 & 1.302 & 33.76 & 0.308 & - & - & - \\
\hline $7 \mathrm{H}-4,17$ & 58.77 & 27.63 & 1 & 50.51 & I & 1.355 & 47.96 & 0.389 & - & - & - \\
\hline $7 \mathrm{H}-4,32$ & 58.92 & 21.95 & $i$ & 59.55 & 1 & 1.391 & 56.54 & 0.444 & - & - & - \\
\hline $7 \mathrm{H}-4,47$ & 59.07 & 27.41 & 1 & 55.82 & 1 & 1.359 & 48.95 & 0.395 & - & - & - \\
\hline $7 \mathrm{H}-4,62$ & 59.22 & 22.56 & $i$ & 56.16 & $i$ & 1.356 & 48.19 & 0.391 & - & - & - \\
\hline $7 \mathrm{H}-4,77$ & 59.37 & 23.88 & 2 & 52.37 & 1 & 1.367 & 50.90 & 0.407 & - & - & - \\
\hline $7 \mathrm{H}-4,92$ & 59.52 & 24.13 & 1 & 53.52 & 1 & 1.400 & 58.53 & 0.458 & - & - & - \\
\hline 138-847C- & & & & & & & & & & & \\
\hline $7 \mathrm{H}-1,17$ & 59.17 & 20.12 & 1 & 66.52 & 1 & 1.401 & 58.75 & 0.460 & - & - & - \\
\hline $7 \mathrm{H}-1,30$ & 59.30 & 18.98 & 1 & 66.88 & 1 & 1.448 & 68.35 & 0.532 & - & - & - \\
\hline $7 \mathrm{H}-1.47$ & 59.47 & 23.72 & 1 & 58.37 & 1 & 1.409 & 60.48 & 0.472 & - & - & - \\
\hline $7 \mathrm{H}-1,62$ & 59.62 & 25.88 & $i$ & 59.20 & 1 & 1.388 & 55.82 & 0.440 & - & - & - \\
\hline $7 \mathrm{H}-1,77$ & 59.77 & 26.72 & 1 & 58.67 & $i$ & 1.373 & 52.31 & 0.417 & - & - & - \\
\hline $7 \mathrm{H}-1,92$ & 59.92 & 27.76 & 1 & 55.79 & 1 & 1.391 & 56.49 & 0.444 & - & - & - \\
\hline $7 \mathrm{H}-1,107$ & 60.07 & 30.84 & I & 55.32 & 1 & 1.358 & 48.64 & 0.394 & - & - & - \\
\hline $7 \mathrm{H}-1,122$ & 60.22 & 31.02 & $i$ & 51.94 & $i$ & 1.355 & 47.89 & 0.389 & - & - & - \\
\hline $7 \mathrm{H}-1,137$ & 60.37 & 30.61 & 1 & 53.63 & 1 & 1.360 & 49.13 & 0.397 & - & - & - \\
\hline $7 \mathrm{H}-2,2$ & 60.52 & 32.08 & 1 & 52.13 & 1 & 1.372 & 52.04 & 0.415 & - & - & - \\
\hline $7 \mathrm{H}-2,17$ & 60.67 & 36.48 & 1 & 46.89 & 1 & 1.337 & 43.26 & 0.361 & - & - & - \\
\hline $7 \mathrm{H}-2,30$ & 60.80 & 40.45 & $i$ & 40.27 & $i$ & 1.327 & 40.59 & 0.346 & - & - & - \\
\hline $7 \mathrm{H}-2,47$ & 60.97 & 37.26 & 1 & 42.31 & 1 & 1.328 & 40.86 & 0.347 & - & - & - \\
\hline $7 \mathrm{H}-2,64$ & 61.14 & 40.04 & 1 & 31.97 & $i$ & 1.289 & 29.87 & 0.288 & - & - & - \\
\hline $7 \mathrm{H}-2,77$ & 61.27 & 36.13 & 1 & 38.64 & 1 & 1.318 & 38.13 & 0.332 & - & - & - \\
\hline $7 \mathrm{H}-2,92$ & 61.42 & 35.45 & 1 & 38.33 & 1 & 1.319 & 38.40 & 0.334 & - & - & - \\
\hline $7 \mathrm{H}-2,107$ & 61.57 & 34.76 & 1 & 39.45 & $i$ & 1.327 & 40.57 & 0.346 & - & - & - \\
\hline $7 \mathrm{H}-2.122$ & 61.72 & 37.39 & 1 & 36.69 & 1 & 1.305 & 34.48 & 0.312 & - & - & - \\
\hline $7 \mathrm{H}-2,137$ & 61.87 & 36.83 & i & 30.88 & 1 & 1.313 & 36.72 & 0.324 & - & - & - \\
\hline $7 \mathrm{H}-3,2$ & 62.02 & 34.31 & 1 & 35.30 & 1 & 1.312 & 36.43 & 0.323 & - & - & - \\
\hline $7 \mathrm{H}-3.17$ & 62.17 & 17.06 & 1 & 62.61 & 1 & 1.365 & 50.30 & 0.404 & - & - & - \\
\hline $7 \mathrm{H}-3,30$ & 62.30 & 15.95 & i & 65.91 & $i$ & 1.428 & 64.36 & 0.501 & - & - & - \\
\hline $7 \mathrm{H}-3,47$ & 62.47 & 18.32 & 1 & 58.58 & 1 & 1.383 & 54.57 & 0.432 & - & - & - \\
\hline $7 \mathrm{H}-3,62$ & 62.62 & 20.43 & 1 & 60.47 & 1 & 1.396 & 57.53 & 0.452 & - & - & - \\
\hline $7 \mathrm{H}-3,77$ & 62.77 & 19.92 & 1 & 62.59 & 1 & 1.409 & 60.38 & 0.472 & - & - & - \\
\hline $7 \mathrm{H}-3,92$ & 62.92 & 22.85 & 1 & 48.01 & 1 & 1.352 & 47.04 & 0.384 & - & - & - \\
\hline $7 \mathrm{H}-3,107$ & 63.07 & 25.78 & 1 & 43.91 & $i$ & 1.329 & 41.05 & 0.349 & - & - & - \\
\hline $7 \mathrm{H}-3,122$ & 63.22 & 25.62 & 1 & 50.68 & 1 & 1.398 & 57.96 & 0.455 & - & - & - \\
\hline $7 \mathrm{H}-3.137$ & 63.37 & 13.30 & 1 & 71.89 & 1 & 1.460 & 70.46 & 0.551 & - & - & - \\
\hline $7 \mathrm{H}-4,2$ & 63.52 & 13.30 & 1 & 70.77 & $i$ & 1.457 & 69.91 & 0.546 & - & - & - \\
\hline $7 \mathrm{H}-4,17$ & 63.67 & 14.15 & 1 & 70.40 & 1 & 1.440 & 66.70 & 0.520 & - & - & - \\
\hline $7 \mathrm{H}-4,30$ & 63.80 & 15.11 & 1 & 68.92 & 1 & 1.441 & 66.89 & 0.521 & - & - & - \\
\hline $7 \mathrm{H}-4,47$ & 63.97 & 18.30 & 1 & 65.63 & $i$ & 1.400 & 58.38 & 0.458 & - & - & - \\
\hline
\end{tabular}


Table 2 (continued).

\begin{tabular}{|c|c|c|c|c|c|c|c|c|c|c|c|}
\hline $\begin{array}{l}\text { Core, section, } \\
\text { interval }(\mathrm{cm})\end{array}$ & $\begin{array}{l}\text { ODP } \\
\text { depth } \\
\text { (mbsf) }\end{array}$ & $\begin{array}{l}\text { Opal } \\
(\%)\end{array}$ & $\begin{array}{c}\text { Analyses } \\
\text { (\#) }\end{array}$ & $\mathrm{CaCO}_{3}$ & $\begin{array}{c}\text { Analyses } \\
\text { (\#) }\end{array}$ & $\begin{array}{c}\text { GRAPE } \\
\text { (smoothed and } \\
\text { interpolated) }\end{array}$ & $\begin{array}{c}\text { Estimated } \\
\mathrm{CaCO}_{3}\end{array}$ & $\begin{array}{l}\text { Dry bulk } \\
\text { density } \\
(\mathrm{g} / \mathrm{cm} 3)\end{array}$ & $\begin{array}{l}\text { Weight } \\
>150 \mu \mathrm{m} \\
(\%)\end{array}$ & $\begin{array}{c}\text { Whole } \\
\text { foraminifers } \\
(\%)\end{array}$ & $\begin{array}{c}\text { Radiolarians } \\
>150 \mu \mathrm{m} \\
(\%)\end{array}$ \\
\hline $7 \mathrm{H}-4,62$ & 64.12 & 17.63 & 1 & 62.55 & 1 & 1.407 & 59.91 & 0.469 & - & - & - \\
\hline $7 \mathrm{H}-4,77$ & 64.27 & 17.52 & 1 & 64.45 & 1 & 1.403 & 59.03 & 0.463 & - & - & - \\
\hline $7 \mathrm{H}-4,92$ & 64.42 & 15.93 & 1 & 68.77 & 1 & 1.451 & 68.78 & 0.537 & - & - & - \\
\hline $7 \mathrm{H}-4,107$ & 64.57 & 14.95 & 1 & 71.46 & 1 & 1.482 & 74.26 & 0.584 & - & - & - \\
\hline $7 \mathrm{H}-4,122$ & 64.72 & 13.26 & $i$ & 74.88 & 1 & 1.472 & 72.56 & 0.569 & - & - & - \\
\hline $7 \mathrm{H}-4,137$ & 64.87 & 11.95 & 1 & 73.70 & 1 & 1.450 & 68.58 & 0.535 & - & - & - \\
\hline $7 \mathrm{H}-5,2$ & 65.02 & 13.84 & 1 & 71.80 & 1 & 1.475 & 73.07 & 0.574 & - & - & - \\
\hline $7 \mathrm{H}-5,17$ & 65.17 & 19.47 & 1 & 63.83 & 1 & 1.442 & 67.05 & 0.523 & - & - & - \\
\hline $7 \mathrm{H}-5,30$ & 65.30 & 19.44 & 1 & 61.21 & 1 & 1.414 & 61.37 & 0.480 & - & - & - \\
\hline $7 \mathrm{H}-5,47$ & 65.47 & 13.82 & i & 74.13 & i & 1.464 & 71.13 & 0.557 & - & - & - \\
\hline $7 \mathrm{H}-5,62$ & 65.62 & 15.79 & 1 & 69.18 & 1 & 1.451 & 68.75 & 0.537 & - & - & - \\
\hline $7 \mathrm{H}-5,77$ & 65.77 & 17.71 & 1 & 63.59 & 1 & 1.429 & 64.46 & 0.503 & - & - & - \\
\hline $7 \mathrm{H}-5,92$ & 65.92 & 19.08 & 1 & 63.73 & 1 & 1.423 & 63.23 & 0.494 & - & - & - \\
\hline $7 \mathrm{H}-5,107$ & 66.07 & 17.68 & 1 & 65.55 & $i$ & 1.442 & 67.02 & 0.523 & - & - & - \\
\hline $7 \mathrm{H}-5,122$ & 66.22 & 13.73 & 1 & 68.52 & 1 & 1.458 & 70.03 & 0.547 & - & - & - \\
\hline $7 \mathrm{H}-5,137$ & 66.37 & 16.14 & 1 & 61.89 & 1 & 1.432 & 65.05 & 0.507 & - & - & - \\
\hline $7 \mathrm{H}-6,2$ & 66.52 & 19.71 & 1 & 63.40 & 1 & 1.405 & 59.40 & 0,466 & - & - & - \\
\hline $7 \mathrm{H}-6,17$ & 66.67 & 21.28 & i & 58.99 & i & 1.402 & 58.74 & 0.461 & - & - & - \\
\hline $7 \mathrm{H}-6,30$ & 66.80 & 22.32 & 1 & 56.91 & 1 & 1.375 & 52.56 & 0.420 & - & - & - \\
\hline $7 \mathrm{H}-6,47$ & 66.97 & 20.72 & 1 & 61.59 & 1 & 1.413 & 61.11 & 0.478 & - & - & - \\
\hline $7 \mathrm{H}-6.64$ & 67.14 & 18.88 & 1 & 63.45 & 1 & 1.433 & 65.23 & 0.509 & - & - & - \\
\hline $7 \mathrm{H}-6,77$ & 67.27 & 17.28 & 1 & 64.61 & 1 & 1.439 & 66.41 & 0.518 & - & - & - \\
\hline $7 \mathrm{H}-6,92$ & 67.42 & 17.85 & 1 & 62.44 & 1 & 1.411 & 60.67 & 0.475 & - & - & - \\
\hline 7H-6. 107 & 67.57 & 17.35 & 1 & 64.28 & 1 & 1.410 & 60.45 & 0.474 & - & - & - \\
\hline $7 \mathrm{H}-6,122$ & 67.72 & 14.56 & 1 & 68.74 & 1 & 1.442 & 66.98 & 0.523 & - & - & - \\
\hline $7 \mathrm{H}-6,137$ & 67.87 & 15.55 & 1 & 65.03 & 1 & 1.467 & 71.61 & 0.561 & - & - & - \\
\hline $7 \mathrm{H}-7,2$ & 68.02 & 19.71 & 1 & 61.59 & 1 & 1.416 & 61.72 & 0.483 & - & - & - \\
\hline $7 \mathrm{H}-7,17$ & 68.17 & 20.67 & 1 & 58.15 & 1 & 1.385 & 54.86 & 0.435 & - & - & - \\
\hline $7 \mathrm{H}-7.30$ & 68.30 & 17.92 & 1 & 64.85 & 1 & 1.420 & 62.55 & 0.489 & - & - & - \\
\hline \multicolumn{12}{|l|}{ 138-847D- } \\
\hline $8 \mathrm{H}-3,122$ & 67.82 & 17.32 & I & 67.37 & 1 & 1.416 & 61.71 & 0.483 & - & - & - \\
\hline $8 \mathrm{H}-3,137$ & 67.97 & 26.92 & 1 & 57.28 & 1 & 1.400 & 58.25 & 0.458 & - & - & - \\
\hline $8 \mathrm{H}-4,2$ & 68.12 & 22.23 & 1 & 63.02 & 1 & 1.404 & 59.12 & 0.464 & - & - & - \\
\hline $8 \mathrm{H}-4,17$ & 68.27 & 22.49 & i & 64.21 & 1 & 1.425 & 63.57 & 0.497 & - & - & - \\
\hline $8 \mathrm{H}-4,32$ & 68.42 & 23.61 & 1 & 64.43 & 1 & 1.419 & 62.32 & 0.487 & - & - & - \\
\hline $8 \mathrm{H}-4,47$ & 68.57 & 24.32 & 1 & 61.34 & 1 & 1.401 & 58.45 & 0.460 & - & - & - \\
\hline $8 \mathrm{H}-4,62$ & 68.72 & 22.33 & 1 & 60.98 & 1 & 1.390 & 55.97 & 0.443 & - & - & - \\
\hline $8 \mathrm{H}-4,77$ & 68.87 & 20.32 & 1 & 62.08 & 1 & 1.397 & 57.55 & 0.454 & - & - & - \\
\hline $8 \mathrm{H}-4,92$ & 69.02 & 21.01 & 1 & 63.12 & 1 & 1.425 & 63.54 & 0.497 & - & - & - \\
\hline $8 \mathrm{H}-4,107$ & 69.17 & 16.62 & 1 & 70.33 & 1 & 1.456 & 69.57 & 0.544 & - & - & - \\
\hline $8 \mathrm{H}-4,122$ & 69.32 & 18.06 & 1 & 69.70 & 1 & 1.439 & 66.34 & 0.518 & - & - & - \\
\hline $8 \mathrm{H}-4,137$ & 69.47 & 25.98 & 1 & 58.27 & 1 & 1.396 & 57.31 & 0.452 & - & - & - \\
\hline $8 \mathrm{H}-5,2$ & 69.62 & 25.88 & 1 & 53.94 & 1 & 1.419 & 62.29 & 0.487 & - & - & - \\
\hline $8 \mathrm{H}-5,17$ & 69.77 & 26.71 & 1 & 57.68 & 1 & 1.412 & 60.80 & 0.477 & - & - & - \\
\hline $8 \mathrm{H}-5,32$ & 69.92 & 24.25 & 1 & 54.68 & 1 & 1.416 & 61.65 & 0.483 & - & - & - \\
\hline $138-847 \mathrm{C}$ - & & & & & & & & & & & \\
\hline $8 \mathrm{H}-2,2$ & 70.02 & 25.67 & 1 & 62.18 & 1 & 1.394 & 56.84 & 0.449 & - & - & - \\
\hline $8 \mathrm{H}-2,17$ & 70.17 & 24.05 & 1 & 59.54 & 1 & 1.407 & 59.72 & 0.469 & - & - & - \\
\hline $8 \mathrm{H}-2,32$ & 70.32 & 22.59 & 1 & 64.36 & 1 & 1.458 & 69.92 & 0.547 & - & - & - \\
\hline $8 \mathrm{H}-2,47$ & 70.47 & 24.98 & 1 & 60.11 & 1 & 1.425 & 63.51 & 0.497 & - & - & - \\
\hline $8 \mathrm{H}-2,62$ & 70.62 & 22.24 & 1 & 66.67 & 1 & 1.423 & 63.09 & 0.494 & - & - & - \\
\hline $8 \mathrm{H}-2,78$ & 70.78 & 18.94 & 1 & 68.05 & 1 & 1.451 & 68.61 & 0.537 & - & - & - \\
\hline $8 \mathrm{H}-2,92$ & 70.92 & 20.18 & 1 & 67.49 & 1 & 1.458 & 69.90 & 0.547 & - & - & - \\
\hline $8 \mathrm{H}-2,107$ & 71.07 & 21.72 & 1 & 64.76 & 1 & 1.442 & 66.88 & 0.523 & - & - & - \\
\hline $8 \mathrm{H}-2,122$ & 71.22 & 22.30 & 1 & 66.77 & 1 & 1.421 & 62.66 & 0.491 & - & - & - \\
\hline $8 \mathrm{H}-2,137$ & 71.37 & 19.86 & 1 & 68.42 & 1 & 1.457 & 69.71 & 0.546 & - & - & - \\
\hline $8 \mathrm{H}-3,2$ & 71.52 & 17.14 & 1 & 72.85 & 1 & 1.509 & 78.37 & 0.626 & - & - & - \\
\hline $8 \mathrm{H}-3,17$ & 71.67 & 18.72 & 1 & 72.37 & 1 & 1.486 & 74.75 & 0.591 & - & - & - \\
\hline $8 \mathrm{H}-3,32$ & 71.82 & 20.94 & 1 & 71.43 & 1 & 1.459 & 70.06 & 0.549 & - & - & - \\
\hline $8 \mathrm{H}-3,47$ & 71.97 & 19.95 & 1 & 69.25 & 1 & 1.457 & 69.69 & 0.546 & - & - & - \\
\hline $8 \mathrm{H}-3,62$ & 72.12 & 18.61 & I & 70.07 & 1 & 1.465 & 71.14 & 0.558 & - & - & - \\
\hline $8 \mathrm{H}-3,78$ & 72.28 & 14.59 & 1 & 75.32 & 1 & 1.478 & 73.40 & 0.578 & - & - & - \\
\hline $8 \mathrm{H}-3,92$ & 72.42 & 22.63 & 1 & 68.15 & 1 & 1.445 & 67.42 & 0.527 & - & - & - \\
\hline $8 \mathrm{H}-3,107$ & 72.57 & 23.90 & 1 & 64.97 & 1 & 1.436 & 65.66 & 0.514 & - & - & - \\
\hline $8 \mathrm{H}-3,122$ & 72.72 & 24.39 & 1 & 64.00 & 1 & 1.422 & 62.82 & 0.492 & - & - & - \\
\hline $8 \mathrm{H}-3,137$ & 72.87 & 26.48 & 1 & 61.12 & 1 & 1.405 & 59.20 & 0.466 & - & - & - \\
\hline $8 \mathrm{H}-4.2$ & 73.02 & 27.25 & 1 & 60.32 & 1 & 1.413 & 60.92 & 0.478 & - & - & - \\
\hline $8 \mathrm{H}-4,17$ & 73.17 & 26.49 & 1 & 59.37 & 1 & 1.411 & 60.49 & 0.475 & - & - & - \\
\hline $8 \mathrm{H}-4,32$ & 73.32 & 21.26 & I & 62.54 & 1 & 1.400 & 58.09 & 0.458 & - & - & - \\
\hline $8 \mathrm{H}-4,47$ & 73.47 & 18.43 & 1 & 66.76 & 1 & 1.443 & 67.01 & 0.524 & - & - & - \\
\hline $8 \mathrm{H}-4.62$ & 73.62 & 21.65 & 1 & 65.02 & 1 & 1.446 & 67.58 & 0.529 & - & - & - \\
\hline $8 \mathrm{H}-4,78$ & 73.78 & 19.50 & 1 & 64.04 & 1 & 1.435 & 65.43 & 0.512 & - & - & - \\
\hline $8 \mathrm{H}-4,92$ & 73.92 & 15.82 & 1 & 71.81 & 1 & 1.469 & 71.80 & 0.564 & - & - & - \\
\hline $8 \mathrm{H}-4,107$ & 74.07 & 14.16 & 1 & 74.11 & 1 & 1.502 & 77.25 & 0.615 & - & - & - \\
\hline $8 \mathrm{H}-4,122$ & 74.22 & 14.25 & 1 & 75.72 & 1 & 1.512 & 78.76 & 0.631 & - & - & - \\
\hline $8 \mathrm{H}-4,137$ & 74.37 & 18.90 & 1 & 66.47 & 1 & 1.438 & 66.01 & 0.517 & - & - & - \\
\hline $8 \mathrm{H}-5,2$ & 74.52 & 21.73 & I & 61.77 & I & 1.443 & 66.98 & 0.524 & - & - & - \\
\hline $8 \mathrm{H}-5,17$ & 74.67 & 20.67 & 1 & 64.79 & 1 & 1.414 & 61.09 & 0.480 & - & - & - \\
\hline $8 \mathrm{H}-5,32$ & 74.82 & 19.99 & I & 66.40 & I & 1.445 & 67.36 & 0.527 & - & - & - \\
\hline $8 \mathrm{H}-5.47$ & 74.97 & 21.82 & 1 & 64.94 & 1 & 1.447 & 67.74 & 0.531 & - & - & - \\
\hline $8 \mathrm{H}-5,62$ & 75.12 & 18.41 & 1 & 66.85 & 1 & 1.455 & 69.24 & 0.543 & - & - & - \\
\hline $8 \mathrm{H}-5.78$ & 75.28 & 18.48 & 1 & 67.24 & 1 & 1.444 & 67.16 & 0.526 & - & - & - \\
\hline $8 \mathrm{H}-5,92$ & 75.42 & 16.00 & 1 & 68.34 & 1 & 1.437 & 65.79 & 0.515 & - & - & - \\
\hline
\end{tabular}


Table 2 (continued).

\begin{tabular}{|c|c|c|c|c|c|c|c|c|c|c|c|}
\hline $\begin{array}{l}\text { Core, section, } \\
\text { interval }(\mathrm{cm})\end{array}$ & $\begin{array}{c}\text { ODP } \\
\text { depth } \\
\text { (mbsf) }\end{array}$ & $\begin{array}{l}\text { Opal } \\
(\%)\end{array}$ & $\begin{array}{c}\text { Analyses } \\
(\#)\end{array}$ & $\mathrm{CaCO}_{3}$ & $\begin{array}{c}\text { Analyses } \\
(\#)\end{array}$ & $\begin{array}{c}\text { GRAPE } \\
\text { (smoothed and } \\
\text { interpolated) }\end{array}$ & $\begin{array}{c}\text { Estimated } \\
\mathrm{CaCO}_{3}\end{array}$ & $\begin{array}{l}\text { Dry bulk } \\
\text { density } \\
(\mathrm{g} / \mathrm{cm} 3)\end{array}$ & $\begin{array}{l}\text { Weight } \\
>150 \mu \mathrm{m} \\
(\%)\end{array}$ & $\begin{array}{c}\text { Whole } \\
\text { foraminifers } \\
(\%)\end{array}$ & $\begin{array}{c}\text { Radiolarians } \\
>150 \mu \mathrm{m} \\
(\%)\end{array}$ \\
\hline $8 \mathrm{H}-5,107$ & 75.57 & 18.10 & 1 & 67.86 & I & 1.434 & 65.19 & 0.511 & - & - & - \\
\hline $8 \mathrm{H}-5,122$ & 75.72 & 24.06 & 1 & 58.17 & 1 & 1.394 & 56.67 & 0.449 & - & - & - \\
\hline $8 \mathrm{H}-5,137$ & 75.87 & 29.67 & 3 & 49.85 & i & 1.353 & 46.84 & 0.386 & - & - & - \\
\hline $8 \mathrm{H}-6,2$ & 76.02 & 35.46 & i & 41.41 & i & 1.359 & 48.34 & 0.395 & - & - & - \\
\hline $8 \mathrm{H}-6,17$ & 76.17 & 15.81 & i & 69.45 & i & 1.443 & 66.94 & 0.524 & - & - & - \\
\hline $8 \mathrm{H}-6,32$ & 76.32 & 13.01 & i & 72.63 & i & 1.474 & 72.62 & 0.572 & - & - & - \\
\hline $8 \mathrm{H}-6,47$ & 76.47 & 14.15 & i & 71.77 & i & 1.450 & 68.27 & 0.535 & - & - & - \\
\hline $8 \mathrm{H}-6,62$ & 76.62 & 14.96 & i & 69.53 & i & 1.435 & 65.36 & 0.512 & - & - & - \\
\hline $8 \mathrm{H}-6,78$ & 76.78 & 14.99 & i & 71.32 & I & 1.481 & 73.80 & 0.583 & - & - & - \\
\hline $8 \mathrm{H}-6,92$ & 76.92 & 15.45 & 1 & 71.62 & i & 1.458 & 69.75 & 0.547 & - & - & - \\
\hline \multicolumn{12}{|l|}{ 138-847D- } \\
\hline $9 \mathrm{H}-3,32$ & 76.42 & 14.86 & 1 & 68.39 & 1 & 1.449 & 68.07 & 0.534 & - & - & - \\
\hline $9 \mathrm{H}-3,47$ & 76.57 & 15.34 & i & 71.27 & i & 1.423 & 62.91 & 0.494 & - & - & - \\
\hline $9 \mathrm{H}-3,62$ & 76.72 & 19.72 & 1 & 66.59 & 1 & 1.416 & 61.44 & 0.483 & - & - & - \\
\hline $9 \mathrm{H}-3,77$ & 76.87 & 22.59 & i & 61.90 & i & 1.385 & 54.56 & 0.435 & - & - & - \\
\hline $9 \mathrm{H}-3,92$ & 77.02 & 16.05 & i & 73.94 & i & 1.443 & 66.90 & 0.524 & - & - & - \\
\hline $9 \mathrm{H}-3,107$ & 77.17 & 12.48 & 1 & 74.56 & 1 & 1.473 & 72.41 & 0.571 & - & - & - \\
\hline $9 \mathrm{H}-3,122$ & 77.32 & 13.85 & i & 75.72 & $i$ & 1.444 & 67.09 & 0.526 & - & - & - \\
\hline $9 \mathrm{H}-3,137$ & 77.47 & 17.49 & i & 69.19 & i & 1.448 & 67.85 & 0.532 & - & - & - \\
\hline $9 \mathrm{H}-4,2$ & 77.62 & 22.28 & i & 61.85 & $i$ & 1.440 & 66.30 & 0.520 & - & - & - \\
\hline $9 \mathrm{H}-4,17$ & 77.77 & 30.25 & i & 54.64 & i & 1.394 & 56.59 & 0.449 & - & - & - \\
\hline $9 \mathrm{H}-4.32$ & 77.92 & 18.48 & i & 71.00 & i & 1.398 & 57.49 & 0.455 & - & - & - \\
\hline $9 \mathrm{H}-4,47$ & 78.07 & 14.79 & i & 71.78 & $i$ & 1.458 & 69.71 & 0.547 & - & - & - \\
\hline $9 \mathrm{H}-4,62$ & 78.22 & 15.69 & $i$ & 68.72 & 1 & 1.459 & 69.89 & 0.549 & - & - & - \\
\hline $9 \mathrm{H}-4,77$ & 78.37 & 15.26 & 1 & 74.40 & i & 1.457 & 69.52 & 0.546 & - & - & - \\
\hline $9 \mathrm{H}-4,92$ & 78.52 & 12.02 & 1 & 78.99 & 1 & 1.490 & 75.24 & 0.597 & - & - & - \\
\hline $9 \mathrm{H}-4,107$ & 78.67 & 12.07 & i & 71.87 & $i$ & 1.460 & 70.06 & 0.551 & - & - & - \\
\hline $9 \mathrm{H}-4,122$ & 78.82 & 19.04 & $i$ & 60.84 & 1 & 1.407 & 59.45 & 0.469 & - & - & - \\
\hline $9 \mathrm{H}-4,137$ & 78.97 & 25.76 & i & 57.40 & i & 1.385 & 54.49 & 0.435 & - & - & - \\
\hline $9 \mathrm{H}-5.2$ & 79.12 & 24.84 & i & 58.01 & i & 1.402 & 58.34 & 0.461 & - & - & - \\
\hline \multicolumn{12}{|l|}{$138-847 \mathrm{C}-$} \\
\hline $9 \mathrm{H}-5,17$ & 79.27 & 18.91 & 2 & 66.75 & 1 & 1. 444 & 67.04 & 0.526 & - & - & - \\
\hline $9 \mathrm{H}-\mathrm{I}, 32$ & 78.32 & 24.25 & 1 & 59.75 & 1 & 1.407 & 59.43 & 0.469 & - & - & - \\
\hline $9 \mathrm{H}-1,47$ & 78.47 & 15.40 & i & 66.61 & i & 1.448 & 67.79 & 0.532 & - & - & - \\
\hline $9 \mathrm{H}-1,62$ & 78.62 & 14.03 & $i$ & 70.30 & i & 1.486 & 74.56 & 0.591 & - & - & - \\
\hline $9 \mathrm{H}-1,77$ & 78.77 & 13.90 & i & 69.57 & i & 1.473 & 72.35 & 0.571 & - & - & - \\
\hline $9 \mathrm{H}-1,92$ & 78.92 & 14.16 & 1 & 71.20 & i & 1.468 & 71.46 & 0.563 & - & - & - \\
\hline $9 \mathrm{H}-1,107$ & 79.07 & 17.17 & i & 65.83 & i & 1.459 & 69.84 & 0.549 & - & - & - \\
\hline $9 \mathrm{H}-1,122$ & 79.22 & 20.35 & i & 62.95 & 1 & 1.431 & 64.44 & 0.506 & - & - & - \\
\hline $9 \mathrm{H}-\mathrm{I}, 137$ & 79.37 & 17.26 & $i$ & 63.71 & 1 & 1.442 & 66.61 & 0.523 & - & - & - \\
\hline $9 \mathrm{H}-2,2$ & 79.52 & 12.86 & i & 76.21 & i & 1.522 & 80.07 & 0.646 & - & - & - \\
\hline $9 \mathrm{H}-2,17$ & 79.67 & 11.98 & 1 & 76.42 & i & 1.513 & 78.76 & 0.632 & - & - & - \\
\hline $9 \mathrm{H}-2,32$ & 79.82 & 14.62 & i & 67.30 & $i$ & 1.473 & 72.32 & 0.571 & - & - & - \\
\hline $9 \mathrm{H}-2,47$ & 79.97 & 17.56 & i & 70.93 & i & 1.477 & 73.01 & 0.577 & - & - & - \\
\hline $9 \mathrm{H}-2,62$ & 80.12 & 23.66 & i & 62.78 & 1 & 1.437 & 65.61 & 0.515 & - & - & - \\
\hline $9 \mathrm{H}-2,77$ & 80.27 & 37.48 & 2 & 46.00 & 1 & 1.362 & 48.89 & 0.400 & - & - & - \\
\hline $9 \mathrm{H}-2,92$ & 80.42 & 31.31 & $i$ & 52.54 & i & 1.400 & 57.82 & 0.458 & - & - & - \\
\hline $9 \mathrm{H}-2,107$ & 80.57 & 33.38 & i & 54.02 & 1 & 1.392 & 56.01 & 0.446 & - & - & - \\
\hline $9 \mathrm{H}-2,122$ & 80.72 & 33.61 & i & 54.22 & i & 1.396 & 56.92 & 0.452 & - & - & - \\
\hline $9 \mathrm{H}-2,137$ & 80.87 & 26.55 & 1 & 61.12 & 1 & 1.445 & 67.16 & 0.527 & - & - & - \\
\hline $9 \mathrm{H}-3,2$ & 81.02 & 15.65 & 1 & 71.50 & i & 1.489 & 75.00 & 0.595 & - & - & - \\
\hline $9 \mathrm{H}-3,17$ & 81.17 & 13.53 & i & 74.45 & i & 1.510 & 78.28 & 0.627 & - & - & - \\
\hline $9 \mathrm{H}-3,32$ & 81.32 & 11.62 & 1 & 77.71 & i & 1.526 & 80.59 & 0.652 & - & - & - \\
\hline $9 \mathrm{H}-3,47$ & 81.47 & 11.99 & i & 76.35 & $i$ & 1.505 & 77.52 & 0.620 & - & - & - \\
\hline $9 \mathrm{H}-3,62$ & 81.62 & 11.16 & 1 & 77.05 & 1 & 1.489 & 74.98 & 0.595 & - & - & - \\
\hline $9 \mathrm{H}-3,77$ & 81.77 & 12.58 & 1 & 74.18 & i & 1.500 & 76.74 & 0.612 & - & - & - \\
\hline $9 \mathrm{H}-3,92$ & 81.92 & 13.67 & $i$ & 70.17 & $i$ & 1.487 & 74.65 & 0.592 & - & - & - \\
\hline $9 \mathrm{H}-3,107$ & 82.07 & 9.92 & 1 & 74.19 & 2 & 1.531 & 81.27 & 0.660 & - & - & - \\
\hline $9 \mathrm{H}-3,122$ & 82.22 & 10.82 & i & 75.07 & $i$ & 1.526 & 80.58 & 0.652 & - & - & - \\
\hline $9 \mathrm{H}-3,137$ & 82.37 & 8.68 & 1 & 75.33 & 1 & 1.520 & 79.73 & 0.643 & - & - & - \\
\hline $9 \mathrm{H}-4,2$ & 82.52 & 8.47 & i & 76.94 & i & 1.580 & 87.13 & 0.735 & - & - & - \\
\hline $9 \mathrm{H}-4,17$ & 82.67 & 8.20 & 1 & 77.57 & i & 1.508 & 77.95 & 0.624 & - & - & - \\
\hline $9 \mathrm{H}-4,32$ & 82,82 & 9.13 & i & 78.18 & 1 & 1.537 & 82.06 & 0.669 & - & - & - \\
\hline $9 \mathrm{H}-4,47$ & 82.97 & 11.57 & 1 & 73.01 & 1 & 1.515 & 78.99 & 0.635 & - & - & - \\
\hline $9 \mathrm{H}-4,62$ & 83.12 & 16.16 & 1 & 65.62 & 1 & 1.450 & 68.05 & 0.535 & - & - & - \\
\hline $9 \mathrm{H}-4,77$ & 83.27 & 17.82 & i & 62.36 & i & 1.432 & 64.53 & 0.507 & - & - & - \\
\hline $9 \mathrm{H}-4,92$ & 83.42 & 9.85 & 1 & 76.19 & 1 & 1.496 & 76.07 & 0.606 & - & - & - \\
\hline $9 \mathrm{H}-4,107$ & 83.57 & 9.45 & 1 & 78.41 & i & 1.527 & 80.69 & 0.654 & - & - & - \\
\hline $9 \mathrm{H}-4,122$ & 83.72 & 9.08 & 1 & 79.17 & 1 & 1.526 & 80.55 & 0.652 & - & - & - \\
\hline $9 \mathrm{H}-4,137$ & 83.87 & 10.20 & i & 78.06 & i & 1.531 & 81.23 & 0.660 & - & - & - \\
\hline $9 \mathrm{H}-5,2$ & 84.02 & 10.37 & i & 77.16 & i & 1.548 & 83.45 & 0.686 & - & - & - \\
\hline $9 \mathrm{H}-5,17$ & 84.17 & 10.60 & i & 74.24 & i & 1.509 & 78.07 & 0.626 & - & - & - \\
\hline $9 \mathrm{H}-5,32$ & 84.32 & 11.96 & 2 & 76.37 & $\mathrm{i}$ & 1.504 & 77.31 & 0.618 & - & - & - \\
\hline $9 \mathrm{H}-5,47$ & 84.47 & 13.34 & 1 & 73.13 & 1 & 1.503 & 77.15 & 0.617 & - & - & - \\
\hline $9 \mathrm{H}-5,62$ & 84.62 & 16.02 & 1 & 69.24 & 1 & 1.459 & 69.70 & 0.549 & - & - & - \\
\hline $9 \mathrm{H}-5,77$ & 84.77 & 15.40 & $i$ & 72.04 & $i$ & 1.502 & 76.99 & 0.615 & - & - & - \\
\hline $9 \mathrm{H}-5,92$ & 84.92 & 12.22 & 1 & 75.59 & i & 1.521 & 79.82 & 0.644 & - & - & - \\
\hline $9 \mathrm{H}-5,107$ & 85.07 & 13.19 & i & 71.61 & $\mathrm{i}$ & 1.500 & 76.67 & 0.612 & - & - & - \\
\hline $9 \mathrm{H}-5,122$ & 85.22 & 15.11 & I & 68.83 & 1 & 1.460 & 69.87 & 0.551 & - & - & - \\
\hline $9 \mathrm{H}-5,137$ & 85.37 & 17.59 & i & 64.86 & i & 1.462 & 70.23 & 0.554 & - & - & - \\
\hline $9 \mathrm{H}-6,2$ & 85.52 & 17.25 & i & 68.79 & i & 1.474 & 72.36 & 0.572 & - & - & - \\
\hline
\end{tabular}


Table 2 (continued).

\begin{tabular}{|c|c|c|c|c|c|c|c|c|c|c|c|}
\hline $\begin{array}{l}\text { Core, section, } \\
\text { interval }(\mathrm{cm})\end{array}$ & $\begin{array}{l}\text { ODP } \\
\text { depth } \\
\text { (mbsf) }\end{array}$ & $\begin{array}{l}\text { Opal } \\
(\%)\end{array}$ & $\begin{array}{c}\text { Analyses } \\
\text { (\#) }\end{array}$ & $\mathrm{CaCO}_{3}$ & $\begin{array}{c}\text { Analyses } \\
\text { (\#) }\end{array}$ & $\begin{array}{c}\text { GRAPE } \\
\text { (smoothed and } \\
\text { interpolated) }\end{array}$ & $\begin{array}{l}\text { Estimated } \\
\mathrm{CaCO}_{3}\end{array}$ & $\begin{array}{l}\text { Dry bulk } \\
\text { density } \\
(\mathrm{g} / \mathrm{cm} 3)\end{array}$ & $\begin{array}{c}\text { Weight } \\
>150 \mu \mathrm{m} \\
(\%)\end{array}$ & $\begin{array}{c}\text { Whole } \\
\text { foraminifers } \\
(\%)\end{array}$ & $\begin{array}{c}\text { Radiolarians } \\
>150 \mu \mathrm{m} \\
(\%)\end{array}$ \\
\hline $9 \mathrm{H}-6,17$ & 85.67 & 17.09 & 1 & 66.19 & 1 & 1.479 & 73.22 & 0.580 & - & - & - \\
\hline $9 \mathrm{H}-6,32$ & 85.82 & 15.71 & 1 & 70.82 & i & 1.460 & 69.85 & 0.551 & - & - & - \\
\hline $9 \mathrm{H}-6,47$ & 85.97 & 15.58 & i & 71.30 & 1 & 1.467 & 71.11 & 0.561 & - & - & - \\
\hline $9 \mathrm{H}-6,62$ & 86.12 & 17.75 & $i$ & 68.59 & i & 1.449 & 67.79 & 0.534 & - & - & - \\
\hline $9 \mathrm{H}-6,77$ & 86.27 & 17.09 & 1 & 68.89 & 1 & 1.476 & 72.69 & 0.575 & - & - & - \\
\hline $9 \mathrm{H}-6,92$ & 86.42 & 13.12 & 1 & 74.58 & 1 & 1.514 & 78.77 & 0.634 & - & - & - \\
\hline $9 \mathrm{H}-6,107$ & 86.57 & 13.52 & $i$ & 73.28 & $i$ & 1.509 & 78.02 & 0.626 & - & - & - \\
\hline $9 \mathrm{H}-6,122$ & 86.72 & 17.14 & 1 & 69.94 & 1 & 1.477 & 72.85 & 0.577 & - & - & - \\
\hline $9 \mathrm{H}-6,137$ & 86.87 & 20.90 & 1 & 63.20 & 1 & 1.436 & 65.23 & 0.514 & - & - & - \\
\hline $9 \mathrm{H}-7,2$ & 87.02 & 18.30 & 1 & 68.79 & 1 & 1.480 & 73.36 & 0.581 & - & - & - \\
\hline $9 \mathrm{H}-7,17$ & 87.17 & 17.69 & $i$ & 67.01 & i & 1.459 & 69.63 & 0.549 & - & - & - \\
\hline $9 \mathrm{H}-7,32$ & 87.32 & 15.78 & I & 74.09 & 1 & 1.487 & 74.52 & 0.592 & - & - & - \\
\hline $9 \mathrm{H}-7,48$ & 87.48 & 16,72 & 1 & 64.71 & 1 & 1.432 & 64.41 & 0.507 & - & - & - \\
\hline $9 \mathrm{H}-7,62$ & 87.62 & 17.44 & 1 & 61.51 & 1 & 1.425 & 62.98 & 0.497 & - & - & - \\
\hline $9 \mathrm{H}-7.77$ & 87.77 & 15.26 & 1 & 68.05 & 1 & 1.450 & 67.93 & 0.535 & - & - & - \\
\hline
\end{tabular}

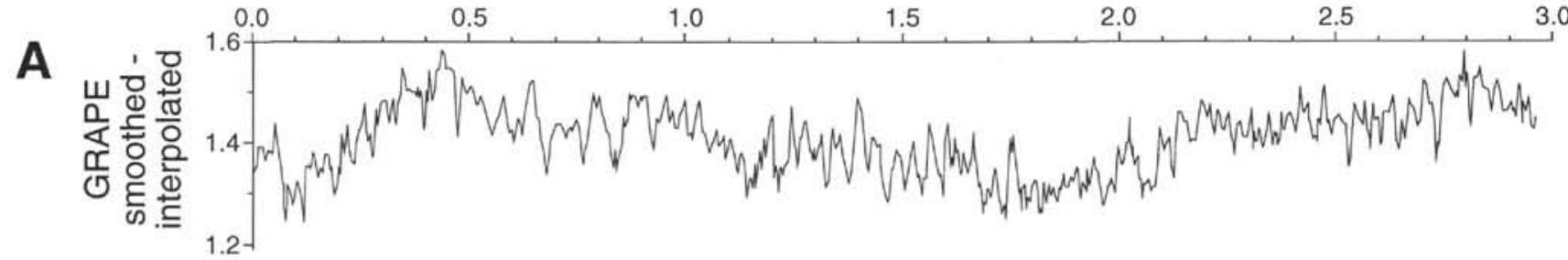

B

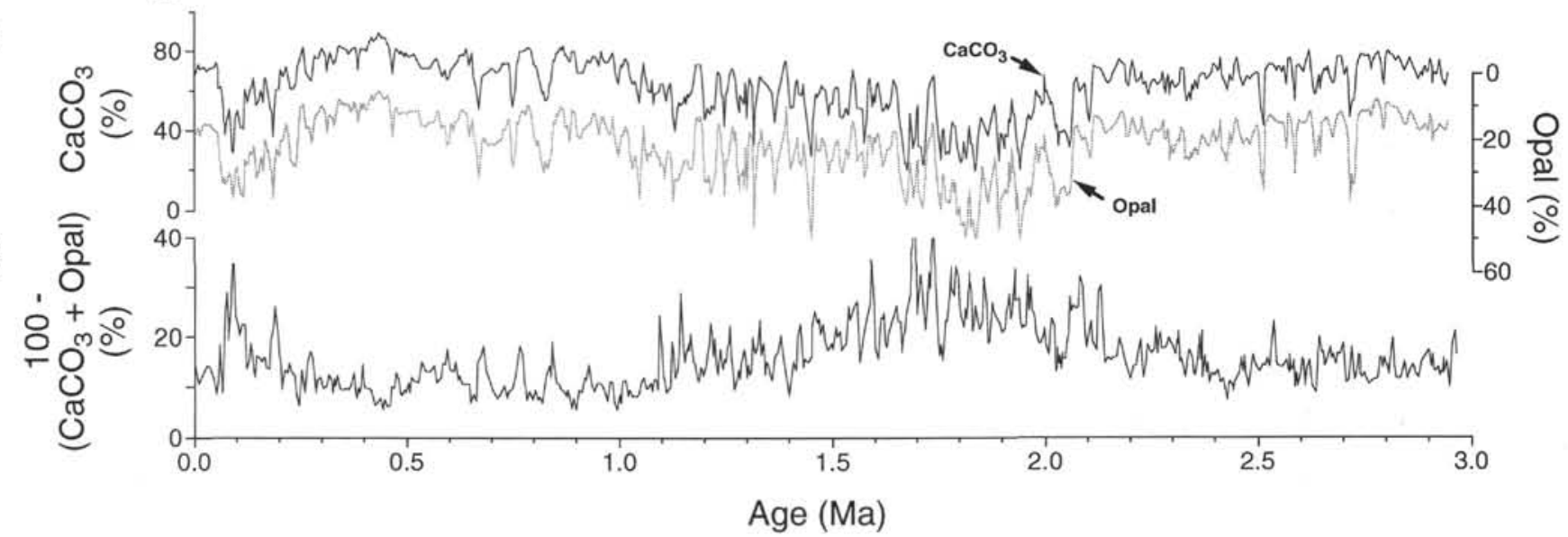

Figure 7. A. Time series for the past $3 \mathrm{~m} . \mathrm{y}$. of GRAPE density data smoothed with a nine-point Gaussian filter and sampled at depths corresponding to samples used in this study. B. \% CaCO 3 and \%opal. C. \%Lithogenic estimated from $\left[100-\left(\mathrm{CaCO}_{3}+\right.\right.$ opal $\left.)\right]$. Note that the opal scale has been inverted. The ages were derived from the GRAPE-based age model of Shackleton et al. (this volume).

estimating calcium carbonate concentrations from the GRAPE density values in nearby piston cores and DSDP sites. This empirical relationship was applied to Leg 138 sites in the study of spatial and temporal variability of carbonate by Hagelberg et al. (this volume). Here, we apply the empirical relationship of Mayer (1991) to the smoothed GRAPE data to estimate carbonate concentrations for the samples analyzed in this study (Table 2). The smoothed data were used because the raw GRAPE data have substantial high-frequency variability, which induces a greater scatter in the estimated $\mathrm{CaCO}_{3}$ concentrations. The estimated carbonate stratigraphy matches the pattern of measured carbonate rather well ( $r=0.93$; Figs. 9A-9B), and the mean of the absolute value of the difference of the measured and estimated values is $4.5 \%$. Some large differences do occur, especially on rapid transitions. The GRAPE data were interpolated to obtain values corresponding to samples used in this study, and this interpolation may contribute to the offsets. GRAPE density and carbonate data had a different sample spacing, and linear interpolation between the adjacent $\sim 2-\mathrm{cm}$-spaced GRAPE measurements was performed to obtain values for comparing with the carbonate measurements. In addition, GRAPE measurements were performed on cores before they were split, whereas sampling for the chemical measurements was performed after they had been split.
Depth offsets resulting from core curation may also be responsible for these large differences.

The differences of the estimated and measured carbonate values were compared to the opal and calcium carbonate concentration data to determine whether the differences can be attributed to either of these components. The estimate of carbonate from GRAPE data assumes that much of the variability results from changes in the relative proportions and packing characteristics of carbonate and opal (Herbert and Mayer, 1991). Should carbonate or opal components at Site 847 have different characteristics from those sites to the west, where the empirical relationships were developed and first applied, the offsets might correlate with either carbonate or opal concentrations. However, no correlation can be seen between the residuals and either carbonate or opal concentrations (Figs. 9C-9D).

Because of its relatively close proximity to South America, Site 847 is likely to have more terrigenous influx than sites to the west. The influx of substantial amounts of this third component is likely to induce errors in the predictions. We used $\left[100-\left(\mathrm{CaCO}_{3}+\right.\right.$ opal $\left.)\right]$ as a measure of the detrital influx (Fig. 7C). The magnitude of the terrigenous values estimated from this method is twice that of estimates based on a sequential leach (S. Hovan, pers. comm., 1992). As 
Table 3. Composite depth reconstruction for Site 847.

\begin{tabular}{|c|c|c|c|c|c|}
\hline $\begin{array}{c}\text { Patch } \\
\text { no. }\end{array}$ & $\begin{array}{l}\text { Core, section, } \\
\text { interval }(\mathrm{cm})\end{array}$ & $\begin{array}{c}\text { ODP } \\
\text { depth } \\
\text { (mbsf) }\end{array}$ & $\begin{array}{l}\text { Interval } \\
\text { length } \\
\text { (m) }\end{array}$ & $\begin{array}{l}\text { Shipboard } \\
\text { composite } \\
\text { (mcd) }\end{array}$ & $\begin{array}{l}\text { Composite } \\
\text { this study } \\
\text { (Bmed) }\end{array}$ \\
\hline \multirow[t]{2}{*}{1} & $138-847 \mathrm{~B}-1 \mathrm{H}-1,0$ to $138-847 \mathrm{~B}-1 \mathrm{H}-3,47$ & $0.00-3.47$ & 3.47 & $0.00-3.47$ & $0.00-3.47$ \\
\hline & $138-847 \mathrm{C}-1 \mathrm{H}-1,122$ to $138-847 \mathrm{C}-1 \mathrm{H}-7,47$ & $3.22-11.47$ & 8.25 & $3.62-11.87$ & $3.50-11.75$ \\
\hline \multirow[t]{2}{*}{2} & 138-847D-2H-3, 62 to $138-847 \mathrm{D}-2 \mathrm{H}-4,32$ & $10.22-11.42$ & 1.20 & $12.02-13.22$ & $11.76-12.96$ \\
\hline & $138-847 \mathrm{C}-2 \mathrm{H}-\mathrm{I}, 17$ to $138-847 \mathrm{C}-2 \mathrm{H}-7,30$ & $11.67-20.80$ & 9.13 & $13.35-22.48$ & $13.08-22.21$ \\
\hline \multirow[t]{2}{*}{3} & 138-847D-3H-3, 62 to 138-847D-3H-3, 137 & $19.72-20.47$ & 0.75 & $22.72-23.47$ & $22.38-23.13$ \\
\hline & $138-847 \mathrm{C}-3 \mathrm{H}-\mathrm{I}, 17$ to $138-847 \mathrm{C}-3 \mathrm{H}-7,62$ & $21.17-30.62$ & 9.45 & $23.57-33.02$ & $23.31-32.76$ \\
\hline \multirow[t]{2}{*}{4} & 138-847D-4H-3, 47 to $138-847 \mathrm{D}-4 \mathrm{H}-3,137$ & $29.07-29.97$ & 0.90 & $33.07-33.97$ & $32.86-33.76$ \\
\hline & $138-847 \mathrm{C}-4 \mathrm{H}-1,32$ to $138-847 \mathrm{C}-4 \mathrm{H}-7,47$ & $30.82-39.97$ & 9.15 & $34.12-43.27$ & $33.94-43.09$ \\
\hline \multirow[t]{2}{*}{5} & 138-847D-5H-3, 107 to 138-847D-5H-4, 92 & $39.17-40.52$ & 1.35 & $43.37-44.72$ & $43.17-44.52$ \\
\hline & $138-847 \mathrm{C}-5 \mathrm{H}-1,47$ to $138-847 \mathrm{C}-5 \mathrm{H}-7.64$ & $40.47-49.64$ & 9.17 & $44.97-54.14$ & $44.62-53.79$ \\
\hline \multirow[t]{2}{*}{6} & $138-847 \mathrm{D}-6 \mathrm{H}-3,32$ to $138-847 \mathrm{D}-6 \mathrm{H}-3,137$ & $47.92-48.97$ & 1.05 & $54.12-55.17$ & $53.89-54.94$ \\
\hline & $138-847 \mathrm{C}-6 \mathrm{H}-1,17$ to $138-847 \mathrm{C}-6 \mathrm{H}-7,17$ & $49.67-58.67$ & 9.00 & $55.37-64.37$ & $54.95-63.95$ \\
\hline \multirow[t]{2}{*}{7} & $138-847 \mathrm{D}-7 \mathrm{H}-3,17$ to $138-847 \mathrm{D}-7 \mathrm{H}-4,92$ & $57.27-59.52$ & 2.25 & $64.47-66.72$ & $64.05-66.30$ \\
\hline & $138-847 \mathrm{C}-7 \mathrm{H}-1.17$ to $138-847 \mathrm{C}-7 \mathrm{H}-7,30$ & $59.17-68.30$ & 9.13 & $66.97-76.10$ & $66.32-75.45$ \\
\hline \multirow[t]{2}{*}{8} & 138-847D-8H-3, 122 to $138-847 \mathrm{D}-8 \mathrm{H}-5,32$ & $67.82-69.92$ & 2.10 & $76.12-78.22$ & $75.55-77.65$ \\
\hline & $138-847 \mathrm{C}-8 \mathrm{H}-2,2$ to $138-847 \mathrm{C}-8 \mathrm{H}-6,92$ & $70.02-76.92$ & 6,90 & $78.22-85.12$ & $77.75-84.65$ \\
\hline \multirow[t]{2}{*}{9} & 138-847D-9H-3, 32 to $138-847 \mathrm{D}-9 \mathrm{H}-5,17$ & $76.42-79.27$ & 2.85 & $85.12-87.97$ & $84.85-87.70$ \\
\hline & $138-847 \mathrm{C}-9 \mathrm{H}-1,32$ to $138-847 \mathrm{C}-9 \mathrm{H}-7,77$ & $78.32-87.77$ & 9.45 & $88.22-97.67$ & $87.87-97.32$ \\
\hline
\end{tabular}

Table 4. GRAPE-based age model from Shackleton et al. (this volume).

\begin{tabular}{|c|c|c|c|c|c|}
\hline $\begin{array}{c}\text { Composite } \\
\text { depth } \\
\text { (rmcd) }\end{array}$ & $\begin{array}{c}\text { Composite } \\
\text { depth } \\
\text { (Bmcd) }\end{array}$ & $\begin{array}{l}\text { Age } \\
\text { (Ma) }\end{array}$ & $\begin{array}{l}\text { Composite } \\
\text { depth } \\
\text { (rmcd) }\end{array}$ & $\begin{array}{l}\text { Composite } \\
\text { depth } \\
\text { (Bmcd) }\end{array}$ & $\begin{array}{l}\text { Age } \\
(\mathrm{Ma})\end{array}$ \\
\hline 0.000 & 0.000 & 0.000 & 55.870 & 55.450 & 1.750 \\
\hline 1.110 & 1.110 & 0.056 & 57.650 & 57.240 & 1.811 \\
\hline 2.730 & 2.730 & 0.082 & 58.790 & 58.370 & 1.832 \\
\hline 3.470 & 3.470 & 0.103 & 61.250 & 60.830 & 1.875 \\
\hline 4.110 & 4.060 & 0.126 & 61.930 & 61.510 & 1.904 \\
\hline 7.650 & 7.530 & 0.218 & 63.710 & 63.290 & 1.947 \\
\hline 8.830 & 8.600 & 0.262 & 64.670 & 64.220 & 1.958 \\
\hline 9.790 & 9.515 & 0.290 & 66.150 & 65.730 & 2.003 \\
\hline 10.290 & 10.000 & 0.311 & 66.830 & 66.420 & 2.023 \\
\hline 11.130 & 10.700 & 0.333 & 68.150 & 67.640 & 2.042 \\
\hline 11.810 & 11.520 & 0.354 & 68.590 & 68.010 & 2.052 \\
\hline 12.230 & 12.000 & 0.372 & 70.070 & 69.420 & 2.097 \\
\hline 13.770 & 13.510 & 0.408 & 70.550 & 69.900 & 2.118 \\
\hline 15.190 & 14.920 & 0.462 & 70.830 & 70.180 & 2.129 \\
\hline 16.050 & 15.780 & 0.484 & 71.170 & 70.520 & 2.140 \\
\hline 17.450 & 17.175 & 0.528 & 73.330 & 72.670 & 2.211 \\
\hline 18.230 & 17.960 & 0.577 & 74.030 & 73.380 & 2.233 \\
\hline 20.330 & 20.060 & 0.648 & 74.990 & 74.340 & 2.255 \\
\hline 21.470 & 21.130 & 0.692 & 75.510 & 74.860 & 2.278 \\
\hline 21.710 & 21.330 & 0.712 & 76.130 & 75.550 & 2.305 \\
\hline 22.690 & 22.350 & 0.749 & 77.430 & 76.850 & 2.348 \\
\hline 24.290 & 24.075 & 0.787 & 78.910 & 78.470 & 2.390 \\
\hline 25.150 & 24.890 & 0.824 & 79.690 & 79.285 & 2.422 \\
\hline 28.150 & 27.890 & 0.908 & 80.390 & 80.040 & 2.438 \\
\hline 29.610 & 29.350 & 0.978 & 82.390 & 81.920 & 2.477 \\
\hline 30.430 & 30.170 & 1.000 & 83.570 & 83.100 & 2.521 \\
\hline 31.530 & 31.270 & 1.050 & 84.490 & 84.070 & 2.547 \\
\hline 32.670 & 32.340 & 1.072 & 84.850 & 84.540 & 2.569 \\
\hline 37.510 & 37.265 & 1.205 & 85.910 & 85.640 & 2.592 \\
\hline 38.150 & 37.790 & 1.215 & 86.770 & 86.490 & 2.614 \\
\hline 39.090 & 38.940 & 1.243 & 87.250 & 86.990 & 2.637 \\
\hline 39,770 & 39.670 & 1.272 & 88.830 & 88.460 & 2.683 \\
\hline 40.510 & 40.270 & 1.283 & 89.470 & 89.110 & 2.707 \\
\hline 41.070 & 40.915 & 1.307 & 89.890 & 89.495 & 2.729 \\
\hline 41.730 & 41.480 & 1.327 & 91.110 & 90.760 & 2.754 \\
\hline 42.410 & 42.230 & 1.337 & 91.470 & 91.120 & 2.776 \\
\hline 43.810 & 43.610 & 1.379 & 91.790 & 91.440 & 2.786 \\
\hline 44.750 & 44.545 & 1.400 & 92.450 & 92.100 & 2.798 \\
\hline 45.570 & 45.325 & 1.431 & 93.930 & 93.580 & 2.835 \\
\hline 47.550 & 47.190 & 1.493 & 94.830 & 94.480 & 2.876 \\
\hline 48.590 & 48.240 & 1.528 & 95.410 & 95.060 & 2.904 \\
\hline 49.830 & 49.480 & 1.567 & 96.330 & 95.980 & 2.926 \\
\hline 50.890 & 50.540 & 1.606 & 96.970 & 96.660 & 2.937 \\
\hline 53.090 & 52.850 & 1.697 & 97.230 & 96.855 & 2.949 \\
\hline 55.150 & 54.900 & 1.718 & 97.750 & 97.370 & 2.969 \\
\hline
\end{tabular}

shown in the Appendix, a strong base $(\mathrm{NaOH})$ used in the sequential leach might remove silica from the clays, thus causing one to underestimate the terrigenous portion of the sediments. In addition, incomplete digestion of radiolarians and the accumulation of other sedimentary components, such as oxyhydroxides, salts, and organics, might contribute to some of this difference. These added influxes are likely to shift the concentrations to lower values, but the pattern of change probably would remain the same. A comparison of our esti-

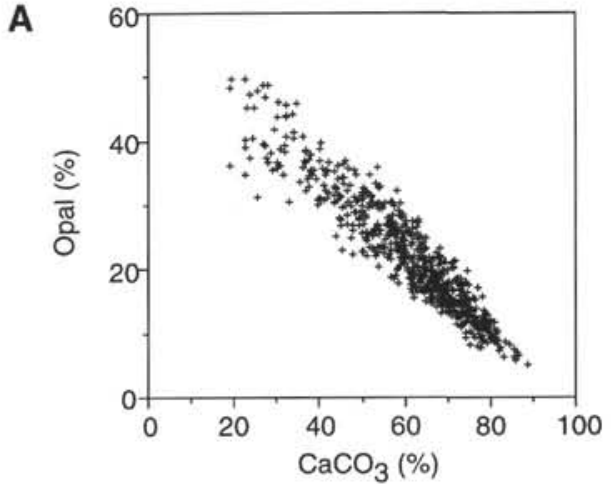

B

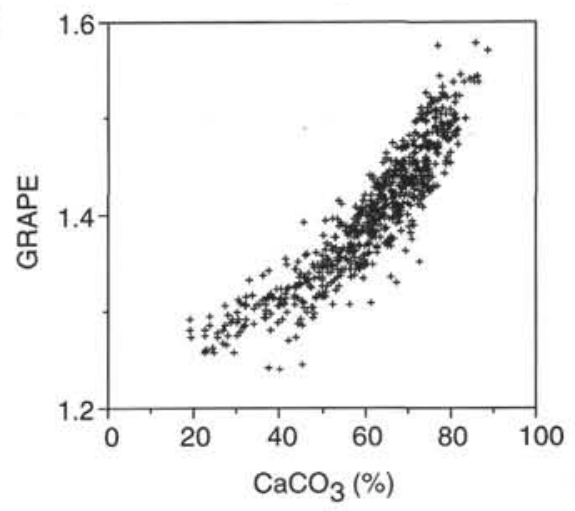

C

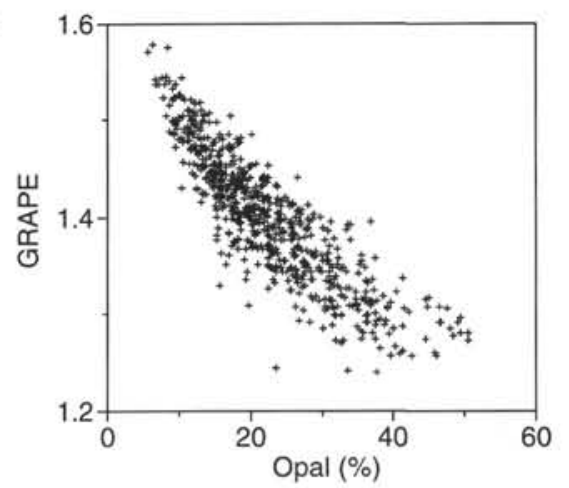

Figure 8. Scatter plots of (A) $\% \mathrm{CaCO}_{3}$ and \%opal, (B) $\% \mathrm{CaCO}_{3}$ and GRAPE, and (C) \%opal and GRAPE data given in Table 2. The opal and carbonate data are highly correlated $(r=-0.94)$. A comparison of GRAPE data to the chemical data reveals a curvilinear relationship, with a large scatter in the high opal range. 
A

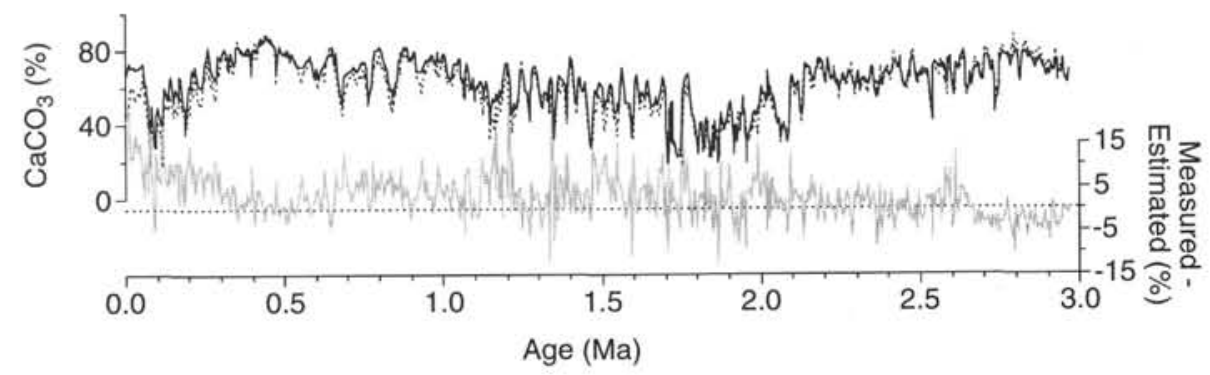

B

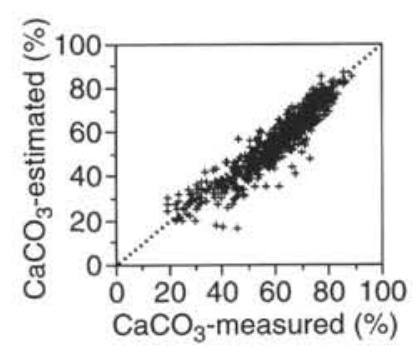

D

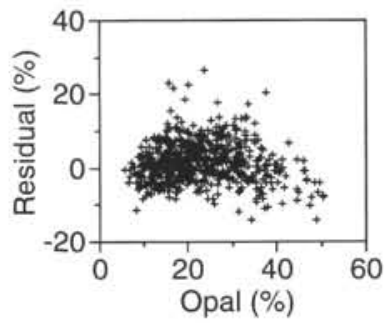

C

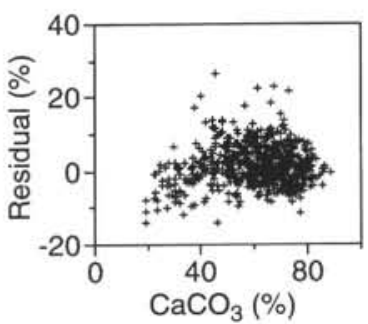

E

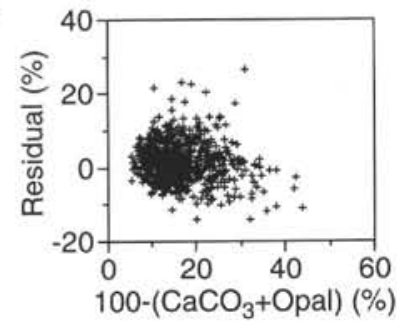

Figure 9. A. Time series of measured $\% \mathrm{CaCO}_{3}$ and values estimated from GRAPE density data using the equations of Mayer (1991) for Cores $1 \mathrm{H}$ to $9 \mathrm{H}$ from Site 847. Also shown is a time series of the difference between measured and estimated values. The largest differences occur on the transitions between high and low values of $\% \mathrm{CaCO}_{3}$. B. Scatter plot of measured vs. estimated $\% \mathrm{CaCO}_{3}$ values. The 1:1 line is shown. C-E. Scatter plots of difference between the measured and estimated $\% \mathrm{CaCO}_{3}$ vs. $\% \mathrm{CaCO}_{3}, \%$ opal, and \%lithogenic as estimated from $\left[100-\left(\mathrm{CaCO}_{3}+\right.\right.$ opal $\left.)\right]$.

mate of the detrital component to the residuals of the carbonate predictions also reveals no correlation (Fig. 9E), and the differences exhibit a random scatter, which may reflect the limits of estimating carbonate from GRAPE data.

\section{Biogenic Sedimentation (0-1 Ma)}

The cyclic changes in carbonate and opal sedimentation in the equatorial Pacific Ocean have been the subject of numerous studies since they were first reported by Arrhenius (1952). During the late Pleistocene, the cycles in central equatorial Pacific locations closely followed the waxing and waning of the ice sheets, implying a response of the equatorial Pacific Ocean to global climatic forcing (Broecker, 1971; Luz and Shackleton, 1975; Pisias and Rea, 1988; Farrell and Prell, 1989). The cyclic changes at Site 847 exhibit some similarities, but also some distinct differences, compared with deeper sites farther to the west. The carbonate concentration changes do exhibit a general pattern of carbonate-rich intervals during glacial periods and carbonate-poor sediments associated with interglacial periods (Fig. 10). However, the amplitude of the signal is less pronounced at Site 847 compared with that at the central equatorial Pacific sites studied by Farrell and Prell (1989). This is to be expected because Site 847 is at or near the top of the sediment lysocline for this area (Berger et al., 1976) and carbonate dissolution, which is a major controlling factor on the amplitude of the central Pacific cycles, should have played a smaller role in altering the carbonate influx to Site 847. The low-carbonate interval during the last major interglacial period $(0.08-0.12 \mathrm{Ma})$ is relatively more pronounced in Site 847 sediments than in sediments from central equatorial Pacific sites. This same event was observed in DSDP Site 572 (Farrell and Prell, 1991), also located near the top of the sediment lysocline for this region (Berger et al., 1976). Dilution by noncarbonate material such as opal may have played a role in the structure of the concentration record. By examining the data in terms of MARs, the effect of dilution should be ameliorated.

The structure in the time series of the MARs is a function of the sedimentation rates and, therefore, the age model used. In Figure 10, we show the time series and spectral characteristics $\delta^{18} \mathrm{O}$ of $G$. sacculifer, GRAPE, $\% \mathrm{CaCO}_{3}$, and $\mathrm{CaCO}_{3} \mathrm{MAR}$ records for the past $1 \mathrm{~m}$.y. using the isotope and GRAPE-based age models. The GRAPE-based chronology assumes that the concentration changes in the equatorial Pacific exhibit the same periodic nature as the Northern Hemisphere insolation changes (Shackleton et al., this volume). The close match between the time series and spectral characteristics of concentration data and oxygen isotopes using the two independent age models implies that the assumption concerning the periodic nature of the concentration changes is generally valid, especially in the low-frequency range (Fig. 10). However, temporal offsets are apparent which will 


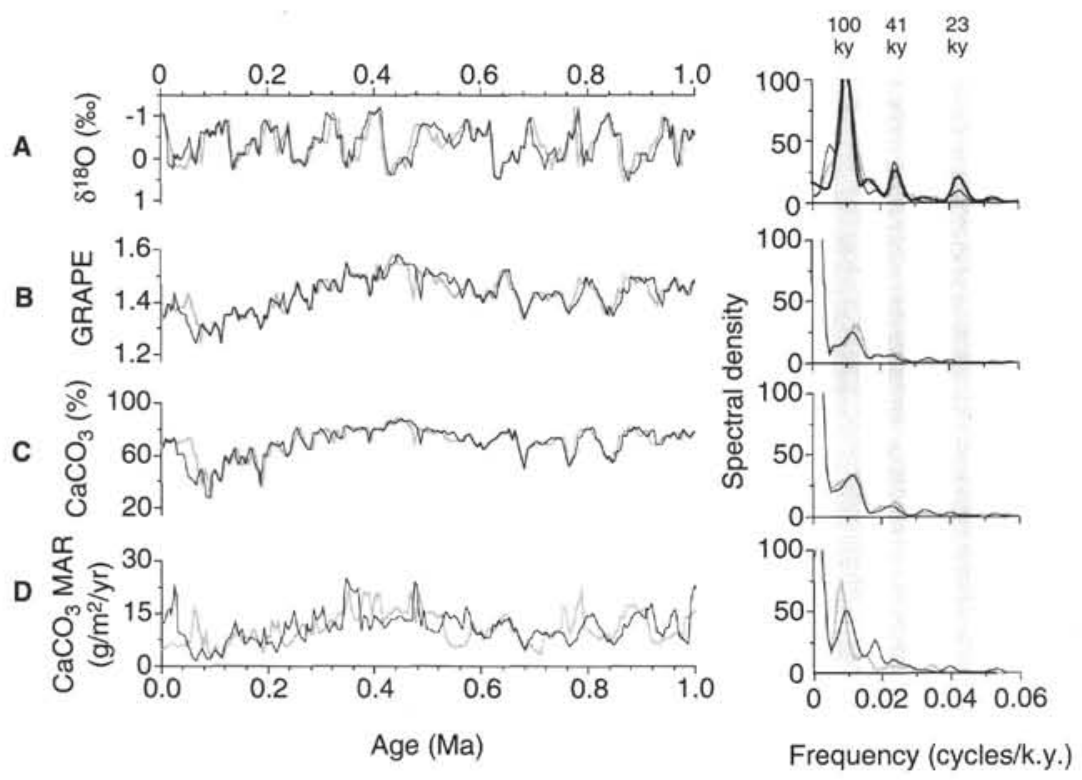

Figure 10. Time series and spectra for (A) $\delta^{18} \mathrm{O}$ of G. sacculifer, (B) GRAPE-smoothed and interpolated, (C) $\% \mathrm{CaCO}_{3}$, and (D) $\mathrm{CaCO}_{3} \mathrm{MAR}$ for the interval from 0 to $1 \mathrm{Ma}$. The ages are based on the G. sacculifer age model of Farrell et al. (this volume) (solid line) and the GRAPE-based, orbitally tuned model of Shackleton et al. (this volume) (thick dotted line). The spectra, plotted as spectral density vs. frequency, were calculated using $\Delta t=4 \mathrm{k} . y ., 90$ lags, and a bandwidth of 0.004 . The dashed line in Figure 10A is the spectra of the SPECMAP stacked isotope time series for the past 0.63 m.y.; 70 lags were used for this analysis. The shaded bands in each spectra depict the 100-, 41-, and 23-k.y. bands associated with variations in the Earth's orbital parameters.

have an impact on the phase estimates between the Site 847 time series using the GRAPE-based age models and records of global climate change (e.g., Imbrie et al., 1992). In addition, these temporal offsets will result in differences in MARs that rely on sedimentation rates derived from these age models (Fig. 10D). The $\mathrm{CaCO}_{3} \mathrm{MAR}$ time series based on the two chronologies are substantially different in the higher frequencies, but the same general trends in the data are apparent in both records (Fig. 10D). The $\mathrm{CaCO}_{3}$ MAR time series using the oxygen isotope-based chronology is strikingly similar to that in the GRAPE and $\% \mathrm{CaCO}_{3}$ records. For the past 1 m.y., the records exhibit a significant near- 100 k.y. periodicity as well as an increase in $\mathrm{CaCO}_{3} \mathrm{MAR}$ between 0.3 and $0.6 \mathrm{Ma}$. The last interglacial period (0.08- $0.12 \mathrm{Ma}$ ) was characterized by low $\mathrm{CaCO}_{3} \mathrm{MAR}$, followed by a substantial increase to high values during the last glacial episode. We examined foraminifer preservation data and the relative influx of siliceous material to identify the cause of these large changes.

The accumulation of $\mathrm{CaCO}_{3}$ should reflect the balance between processes of production and dissolution. The data on the percentage of whole planktonic foraminifers, relative to fragments, provide a relative index of the extent to which dissolution of carbonate has altered the record (Thunell, 1976). The values spanning the last interglacial period range from $47 \%$ to $72 \%$, but show no distinct correlation of high fragmentation during low accumulation (Fig. 11B). In fact, the relative low $\mathrm{CaCO}_{3}$ MARs near 0.07 and $0.09 \mathrm{Ma}$ are associated with a foraminifer assemblage having the same preservation state as during the carbonaterich glacial period (see also McKenna et al, this volume). During this interglacial time interval, the proportion of radiolarians, which reflects the siliceous component of the sediments, relative to foraminifers in the $>150-\mu \mathrm{m}$ fraction is larger than in the following glacial, although the overall accumulation of opal is not greater (Fig. 11C). Taken together, these data imply that the changes are related to the carbonate component and not the opal, and that carbonate production and not dissolution is responsible for the changes in $\mathrm{CaCO}_{3} \mathrm{MAR}$. The production of carbonate was lower during the last interglacial, resulting in a relative increase in the siliceous component of the plankton.

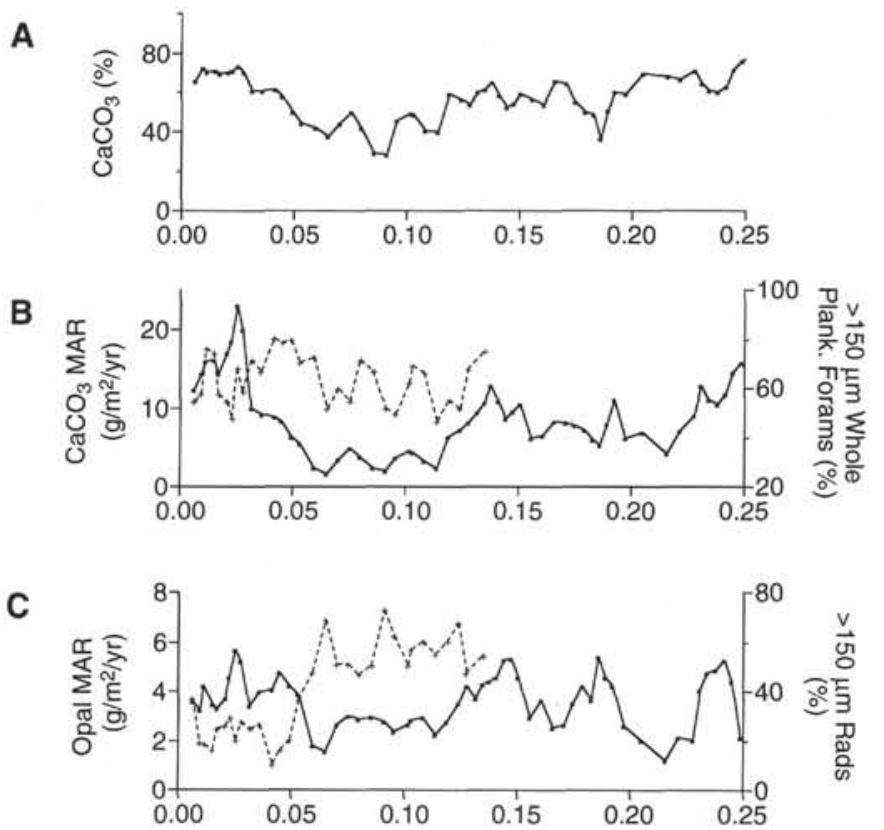

Figure 11. Time series for the past $0.5 \mathrm{~m} . \mathrm{y}$. of Site 847 carbonate and opal data. A. $\% \mathrm{CaCO}_{3}$. B. $\mathrm{CaCO}_{3} \mathrm{MAR}$ (solid line) and percentage of whole planktonic foraminifers $>150 \mu \mathrm{m}$ (dashed line). C. Opal MAR (solid line) and percentage of radiolarians $>150 \mu \mathrm{m}$ relative to foraminifers (dashed line). The ages are based on the oxygen isotope chronostratigraphy.

\section{Biogenic Sedimentation (0-3 Ma)}

Time series of carbonate concentration and $\mathrm{CaCO}_{3}$ and opal MAR for the past 3 m.y. are shown in Figure 12 . The pattern of $\% \mathrm{CaCO}_{3}$ for Site 847 is similar to that observed at DSDP Site 572 , almost $20^{\circ}$ to the west (Farrell and Prell, 1991). For this analysis, we used the age 


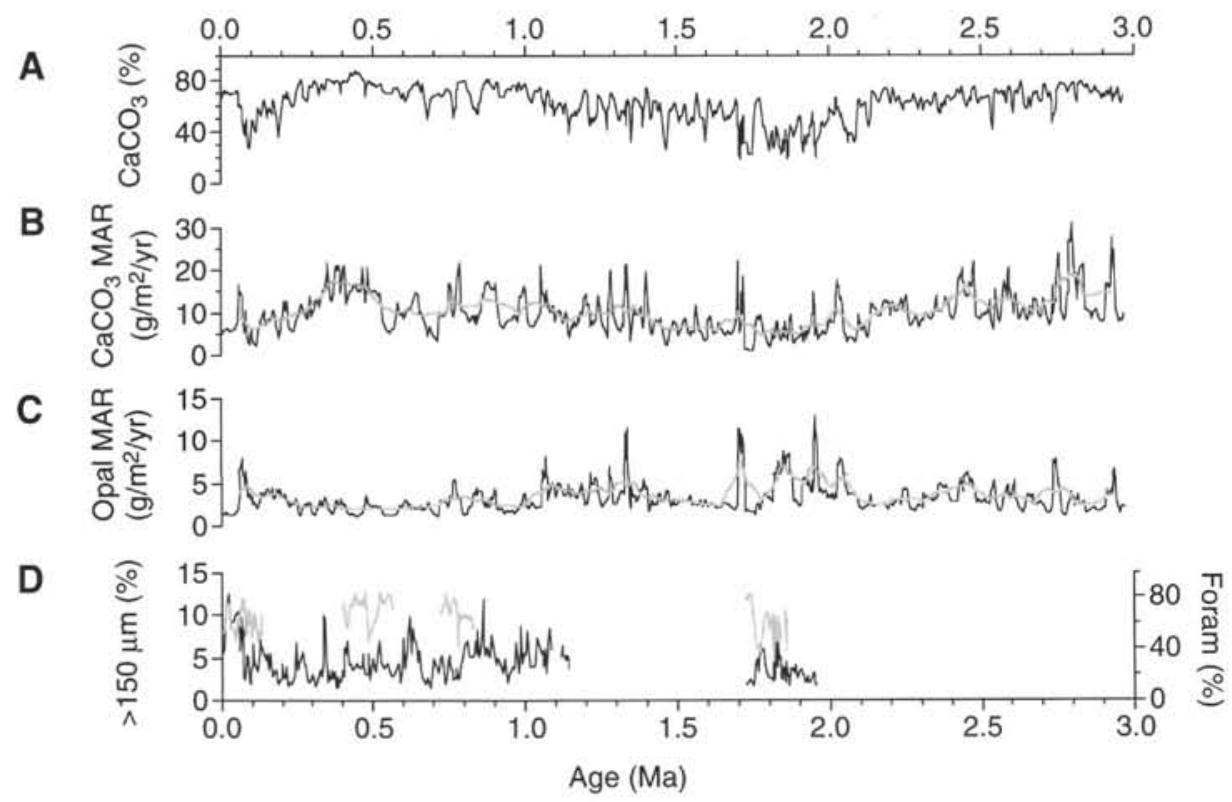

Figure 12. Time series of (A) $\% \mathrm{CaCO}_{3},(\mathbf{B}) \mathrm{CaCO}_{3} \mathrm{MAR}$, (C) opal MAR, and (D) weight $>150 \mu \mathrm{m}$ (solid) and $\%$ foraminifers (thick dotted) for Site 847 spanning the past 3 m.y. The GRAPE-based age model from Shackleton et al. (this volume) was used to provide the chronostratigraphic framework for these time series. The thick dotted lines in Figures 12B and 12C are 20-point running means that capture the major trends in the data.
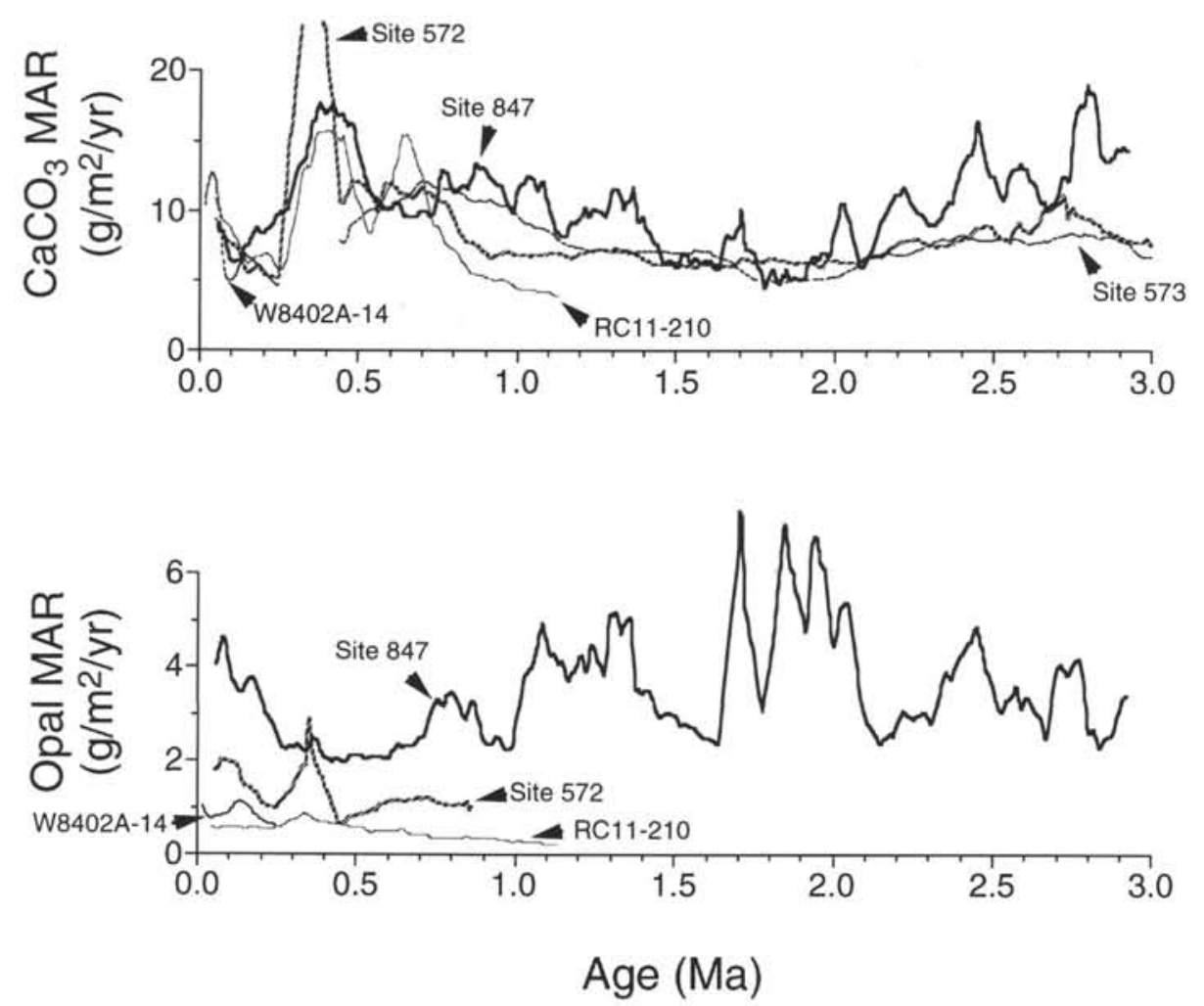

Figure 13. Time series of $\mathrm{CaCO}_{3}$ and opal MARs for sites forming a transect along the equator from $140^{\circ}$ to $95^{\circ} \mathrm{W}$. The plots are 20-point running means of the data for each site to capture the major trends over the past 3 m.y. DSDP Site 573 (thin broken line), Core RC11-210 (thin dotted line), and Core W8402A-14 (thin dashed line) are all located near $140^{\circ} \mathrm{W}$; DSDP Site 572 (thick dotted line) is in the middle near $114^{\circ} \mathrm{W}$; and ODP Site 847 (thick solid line) is in the east near $95^{\circ} \mathrm{W}$ (see text for details). 
model based on tuning the GRAPE data to the summer insolation changes. Therefore, as shown in the previous section, the timing of the higher frequency MAR maxima and minima are suspect, but the longterm trends generally are valid. A 20-point moving average is used to depict the trends in the MAR data for the past 3 m.y. (Fig. 12). High rates of $\mathrm{CaCO}_{3}$ MAR near $2.8 \mathrm{Ma}$ are followed by a distinct minima near $1.8 \mathrm{Ma}$. $\mathrm{CaCO}_{3}$ MAR increases to a maxima near $0.4 \mathrm{Ma}$, followed by a significant decrease in production during the last interglacial period. We used preliminary preservation data to evaluate the relative influence of dissolution on the alteration of the record of carbonate production. In carbonate-rich sediments, where dissolution causes fragmentation of foraminifer tests into smaller particles, coarse fraction data $(>150-\mu \mathrm{m} w t \%)$ have been used as an index of dissolution (Peterson and Prell, 1985). The coarse fraction data from Site 847 (Fig. 12) do not exhibit a correspondence with either the high-frequency or broad trends in the $\mathrm{CaCO}_{3} \mathrm{MAR}$ time series. We concluded that either the coarse fraction is not a reliable index of dissolution for the Site 847 samples or that production is responsible for the structure of the $\mathrm{CaCO}_{3}$ MAR time series. Additional dissolution indexes, such as whole planktonic foraminifers (\%), help to resolve this question. The preliminary data from this index show little correspondence with either the $>150$ $\mu \mathrm{m}(\%)$ or MAR data (Fig. 12). Percentages of whole planktonic foraminifers have a similar range and magnitude within intervals of high and low $\mathrm{CaCO}_{3}$ MAR. Intervals within the low accumulation events of the last interglacial and near $1.8 \mathrm{Ma}$ have relatively high whole-to-fragment proportions, which implies that the minimum in accumulation is related to production rather than dissolution. However, Farrell and Prell (1991) suggested dissolution as the cause of these events at sites farther to the west. Although the data from Site 847 suggest that production controls the long-term changes in carbonate accumulation, additional work using more reliable dissolution indexes will be needed to decipher the cause of these events. Intervals of opal accumulation maxima at times of low $\mathrm{CaCO}_{3}$ MAR (i.e., 1.7-2.0 Ma and 1.1-1.3 Ma) imply that production was dominated by siliceous organisms during these periods, especially because only a small proportion $(\sim 10 \%)$ of the opal rain is presently preserved at these sites (Dymond and Lyle, 1985; Archer et al., 1993).

\section{Equatorial Transect}

A recent compilation of surface primary productivity from the equatorial Pacific Ocean shows a two- to threefold increase in primary production along the equatorial Pacific transect from $140^{\circ}$ to $95^{\circ} \mathrm{W}$ (Berger, 1989). Such a gradient should be reflected in the biogenic components of the underlying sediments. Lyle (1992) showed that the composition of surface sediments in the eastern Pacific does reflect the complex surface productivity gradients in this region near the coast of Central and South America. To evaluate the long-term changes in carbonate and opal productivity along the equator, we selected three sites along an equatorial transect from $140^{\circ}$ to $95^{\circ} \mathrm{W}$ (Fig. 1). Site 847 provides the eastern anchor of the transect. A combination of data from Cores W8402A-14 ( $0^{\circ} 57^{\prime} \mathrm{N}, 138^{\circ} 57^{\prime} \mathrm{W}, 4287 \mathrm{~m}$ water depth), RC11$210\left(1^{\circ} 49^{\prime} \mathrm{N}, 140^{\circ} 03^{\prime} \mathrm{W}, 4420 \mathrm{~m}\right.$ water depth), and DSDP Site 573 $\left(0^{\circ} 30^{\prime} \mathrm{N}, 133^{\circ} 19^{\prime} \mathrm{W}, 4301 \mathrm{~m}\right.$ water depth) are used to quantify the biogenic sedimentation at the western location. The Core RC11-210 data are from Rea et al. (1991), the Core W8402A-14 data are from Lyle et al. (1988), and the DSDP Site 573 carbonate data are from Farrell and Prell (1991). DSDP Site $572\left(1^{\circ} 26^{\prime} \mathrm{N}, 113^{\circ} 51^{\prime} \mathrm{W}, 3903 \mathrm{~m}\right.$ water depth), midway between these sites, completes the transect with carbonate data from Farrell and Prell (1991) and opal data generated as part of this study. All age models were adjusted to be consistent with the orbitally tuned, GRAPE-based chronostratigraphy of Shackleton et al. (this volume). To capture the major trends in the carbonate and opal accumulation rate data for comparisons made in this study, we used a 20-point running mean of each time series (Fig. 13).

The west-to-east gradient in carbonate accumulation is small, especially between 1.5 and $2.0 \mathrm{Ma}$ and during the past 0.5 m.y. One

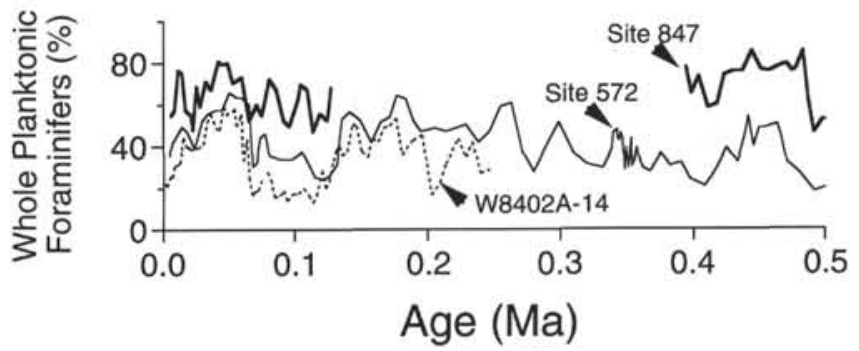

Figure 14. Time series of whole planktonic foraminifers $(\%)$ relative to fragments for the $>150-\mu \mathrm{m}$ fraction of Site W8402A-14 (dashed line, $\sim 139^{\circ} \mathrm{W}$, $4287 \mathrm{~m}$ water depth; Murray, unpubl. data), DSDP Site 572 (thin solid line, $\sim 114^{\circ} \mathrm{W}, 3903 \mathrm{~m}$ water depth; Min-Te Chen, unpubl. data), and Site 847 (thick solid line, $\sim 95^{\circ} \mathrm{W}, 3334 \mathrm{~m}$ water depth; this study).

possibility is that dissolution of carbonate at the eastern sites has altered any preexisting gradient in the flux to the seafloor. Whole planktonic foraminifer (\%) data was used to evaluate the dissolution gradient along the transect. Data for the past $0.5 \mathrm{~m}$.y. show that the carbonate assemblage is better preserved in the eastern Site 847 compared with those in the deeper Sites W8402A-14 and DSDP 572 located farther west (Fig. 14); thus, dissolution cannot account for the small gradient. In fact, these data imply that the initial flux of carbonate before dissolution probably was greater in the central Pacific sites compared with that in the eastern sites. Although the surface gradient in production is not reflected by calcareous plankton, our preliminary data from the opal component show that opal accumulation does exhibit a strong west-to-east gradient during the past 1 m.y. (Fig. 13). Opal accumulation at Site 847 in the east is as much as four to five times the rate of the western Sites W8402A-14 and RC11-210. This factor is greater than the modern productivity gradient. Should opal production track the primary production, the longitudinal opal gradient would highlight the nonlinear relationship between production and preservation of opal in marine sediments, as noted by Broecker and Peng (1982).

\section{CONCLUSIONS}

Sedimentation during the past 3 m.y. at Site 847 , beneath the high productivity region of the equatorial divergence, reflects long-term variations in the production of carbonate- and opal-secreting microplankton in this region. The cyclic changes in carbonate and opal components and their respective packing characteristics cause the density changes observed in the GRAPE WBD records generated by the Leg 138 shipboard scientists. Estimates of $\mathrm{CaCO}_{3}(\%)$ from the GRAPE data provide reliable values, with a mean difference of $4.5 \%$. This difference appears to be random and probably reflects limitation of the GRAPE-based estimates.

A comparison of times series of the $\delta^{18} \mathrm{O}$ of $G$. sacculifer, GRAPE, $\% \mathrm{CaCO}_{3}$, and $\mathrm{CaCO}_{3} \mathrm{MAR}$ for the last $1 \mathrm{~m}$.y. using age models based on oxygen isotope stratigraphy and tuning the GRAPE cycles to summer insolation at $65^{\circ} \mathrm{N}$ reveals that long-term changes are the same with the different models but that high-frequency orbital changes are different. This is especially true for the $\mathrm{CaCO}_{3} \mathrm{MAR}$ time series, where glacial to interglacial changes are age-model dependent.

An evaluation of distinct low-carbonate events during the last interglacial period and near 1.8 Ma suggests that production, not dissolution, is responsible for these changes. The relative increase in opal accumulation within these intervals implies that flux of material to the seafloor was attributed largely to siliceous microplankton, rather than calcareous organisms.

The long-term changes in carbonate and opal accumulation along a transect from the central to eastern equatorial Pacific during the past $3 \mathrm{~m}$.y. show little gradient in carbonate accumulation, even though the carbonate component is better preserved in the eastern site. However, 
a steep gradient in opal accumulation that is greater than the modern productivity changes across the region can be seen. These data highlight the nonlinear relationship between opal production and accumulation, where high rain rates have enhanced preservation.

\section{ACKNOWLEDGMENTS}

This project could not have been completed without the help of the world-class Brown sailors (Mike Zani, Site 847 carbonate and opal; Brian Doyle and Guy Adema, Site 572 opal) who spent numerous hours in the laboratory before the winds picked up. Mel Waldorf helped with sample grinding and Site 847 carbonate analyses before moving on to his pollen studies. We are grateful to Carmen Cors, April Martin, Jenna Cook, and Jane Donnelly for their help in preparation and counts of the faunal samples. Thanks to Min-Te Chen for providing unpublished fragmentation data for Site 572, Terri Hagelberg for providing revised composite depths for the Site 847 GRAPE data, and Nick Shackleton for distributing his orbitally tuned GRAPE-based age model. Technical support for this project was obtained by grants from JOI/USSAC (TAMRF-20528) and NSF/ATM (ATM-8812589).

\section{REFERENCES $*$}

Archer, D., Lyle, M., Rodgers, K., and Froelich, P., 1993. What controls opal preservation in tropical deep-sea sediments? Paleoceanography, 8:7-21.

Archer, D.E., 1991a. Equatorial Pacific calcite preservation cycles: production or dissolution? Paleoceanography, 6:561-571. 17050 .

Arrhenius, G., 1952. Sediment cores from the East Pacific. In Pettersson, H. (Ed.), Rep. Swed. Deep-Sea Exped. 1947-48, 5:1-227.

Berger, W.H., 1989. Global maps of ocean productivity. In Berger, W.H., Smetacek, V.S., and Wefer, G. (Eds.), Productivity of the Oceans: Present and Past: New York (Wiley), 429-455.

Berger, W.H., Adelseck, C.G, and Mayer, L., 1976. Distribution of carbonate in surface sediments of the Pacific Ocean. J. Geophys. Res., 81:2617-2627.

Broecker, W.S., 1971. Calcite accumulation rates and glacial to interglacial changes in oceanic mixing. In Turekian, K.K. (Ed.), The Late Cenozoic Glacial Ages: New Haven, CT (Yale Univ. Press), 239-265.

Broecker, W.S., and Peng, T.-H., 1982. Tracers in the Sea: Palisades, NY (Lamont-Doherty Geological Observatory).

Chavez, F.P., and Barber, R.T., 1987. An estimate of new production in the equatorial Pacific. Deep-Sea Res. Part A, 34:1229-1243.

Clemens, S., Prell, W., Murray, D., Shimmield, G.B., and Weedon, G., 1991. Forcing mechanisms of the Indian Ocean monsoon. Nature, 353:720-725.

Dymond, J., and Lyle, M., 1985. Flux comparisons between sediments and sediment traps in the eastern tropical Pacific: implications for atmospheric $\mathrm{CO}_{2}$ variations during the Pleistocene. Limnol. Oceanogr., 30:699-712.

Farrell, J.W., 1991. Late Neogene palaeoceanography of the central equatorial Pacific: evidence from carbonate preservation and stable isotopes [Ph.D. thesis]. Brown Univ., Providence, RI.

Farrell, J.W., and Janecek, T.R., 1991. Late Neogene paleoceanography and paleoclimatology of the northeast Indian Ocean (Site 758). In Weissel, J., Peirce, J., Taylor, E., Alt, J., et al., Proc. ODP, Sci. Results, 121: College Station, TX (Ocean Drilling Program), 297-358.

Farrell, J.W., and Prell, W.L., 1989. Climatic change and $\mathrm{CaCO}_{3}$ preservation: an 800,000 year bathymetric reconstruction from the central equatorial Pacific Ocean. Paleoceanography, 4:447-466.

1991. Pacific $\mathrm{CaCO}_{3}$ preservation and $\delta^{18} \mathrm{O}$ since $4 \mathrm{Ma}$ : paleoceanic and paleoclimatic implications. Paleoceanography, 6:485-498.

Hagelberg, T., Shackleton, N., Pisias, N., and Shipboard Scientific Party, 1992. Development of composite depth sections for Sites 844 through 854 . In Mayer, L., Pisias, N., Janecek, T., et al., Proc. ODP, Init. Repts., 138 (Pt. 1): College Station, TX (Ocean Drilling Program), 79-85.

Herbert, T.D., and Mayer, L.A., 1991. Long climatic time series from sediment physical property measurements. J. Sediment. Petrol., 61:1089-1108.

Imbrie, J., Boyle, E.A., Clemens, S.C., Duffy, A., Howard, W.R., Kukla, G., Kutzbach, J., Martinson, D.G., McIntyre, A., Mix, A.C., Molfino, B., Morley, J.J., Peterson, L.C., Pisias, N.G., Prell, W.L., Raymo, M.E., Shackleton, N.J., and Toggweiler, J.R., 1992. On the structure and origin of major glaciation cycles, 1. Linear responses to Milankovitch forcing. Paleoceanography, 7:701-738.
Jones, G.A., and Kaiteris, P., 1983. A vacuum-gasometric technique for rapid and precise analysis of calcium carbonate in sediments and soils. $J$. Sediment. Petrol., 53:655-660.

Keigwin, L., 1982. Isotopic paleoceanography of the Caribbean and East Pacific: role of Panama Uplift in late Neogene time. Science, 217:350-353.

Leinen, M., Cwienk, D., Heath, G.R., Biscaye, P.E., Kolla, V., Thiede, J., and Dauphin, J.P., 1986. Distribution of biogenic silica and quartz in recent deep-sea sediments. Geology, 14:199-203.

Luz, B., and Shackleton, N.J., 1975. $\mathrm{CaCO}_{3}$ solution in the tropical East Pacific during the past 130,000 years. In Sliter, W.V., Bé, A.W., and Berger, W.H (Eds.), Dissolution of Deep-Sea Carbonates. Spec. Publ. Cushman Found. Foraminiferal Res., 13:142-150.

Lyle, M., 1992. Composition maps of surface sediments of the eastern tropical Pacific Ocean. In Mayer, L., Pisias, N., Janecek, T., et al., Proc. ODP, Init. Repts., 138 (Pt. 1): College Station, TX (Ocean Drilling Program), 101115.

Lyle, M., Murray, D.W., Finney, B.P., Dymond, J., Robbins, J.M., and Brooksforce, K., 1988. The record of Late Pleistocene biogenic sedimentation in the eastern tropical Pacific Ocean. Paleoceanography, 3:39-59.

Mayer, L., Pisias, N., Janecek, T., et al., 1992. Proc. ODP, Init. Repts., 138 (Pts. 1 and 2): College Station, TX (Ocean Drilling Program).

Mayer, L.A., 1991. Extraction of high-resolution carbonate data for paleoclimate reconstruction. Nature, 352:148-150.

Mortlock, R.A., and Froelich, P.N., 1989. A simple method for the rapid determination of biogenic opal in pelagic marine sediments. Deep-Sea Res. Part A, 36:1415-1426.

Murray, D.W., and Prell, W.L., 1991. Pliocene to Pleistocene variations in calcium carbonate, organic carbon, and opal on the Owen Ridge, northern Arabian Sea. In Prell, W.L., Niitsuma, N., et al., Proc. ODP, Sci. Results, 117: College Station, TX (Ocean Drilling Program), 343-363.

Murray, R.W., and Leinen, M., 1993. Chemical transport to the seafloor of the equatorial Pacific Ocean across a latitudinal transect at $135^{\circ} \mathrm{W}$ : tracking sedimentary major, trace, and rare earth element fluxes at the Equator and the Intertropical Convergence Zone. Geochim. Cosmochim. Acta. 57:4141-4163.

Peterson, L.C., and Prell, W.L., 1985. Carbonate dissolution in Recent sediments of the eastern equatorial Indian Ocean: preservation patterns and carbonate loss above the lysocline. Mar. Geol., 64:259-290.

Pisias, N.G., and Rea, D.K., 1988. Late Pleistocene paleoclimatology of the central Equatorial Pacific: sea surface response to the southeast trade winds. Paleoceanography, 3:21-37.

Rea, D.K., Pisias, N.G., and Newberry, T., 1991. Late Pleistocene paleoclimatology of the central Equatorial Pacific: flux patterns of biogenic sediments. Paleoceanography, 6:227-244.

Robinson, S.G., 1990. Applications for whole-core magnetic susceptibility measurements of deep-sea sediments: Leg 115 results. In Duncan, R.A., Backman, J., Peterson, L.C., et al., Proc. ODP, Sci. Results, 115: College Station, TX (Ocean Drilling Program), 737-771.

Ruddiman, W.F., Raymo, M.E., Martinson, D.G., Clement, B.M., and Backman, J., 1989. Pleistocene evolution: Northern Hemisphere ice sheets and North Atlantic Ocean. Paleoceanography, 4:353-412.

Shackleton, N.J., and Shipboard Scientific Party, 1992. Sedimentation rates: toward a GRAPE density stratigraphy for Leg 138 carbonate sections. In Mayer, L., Pisias, N., Janecek, T., et al., Proc. ODP, Init. Repts., 138 (Pt. 1): College Station, TX (Ocean Drilling Program), 87-91.

Shipboard Scientific Party, 1992. Site 847. In Mayer, L., Pisias, N., Janecek, T., et al., Proc. ODP, Init. Repts., 138 (Pt. 1): College Station, TX (Ocean Drilling Program), 335-393.

Takahashi, K., and Honjo, S., 1983. Radiolarian skeletons: size, weight, sinking speed, and residence time in tropical pelagic oceans. Deep-Sea Res. Part A, 30:543-568.

Thunell, R.C., 1976. Optimum indices of calcium carbonate dissolution in deep-sea sediments. Geology, 4:525-528.

\footnotetext{
Abbreviations for names of organizations and publication titles in ODP reference lists follow the style given in Chemical Abstracts Service Source Index (published by American Chemical Society).
}

\section{Date of initial receipt: 9 June 1993 \\ Date of acceptance: 17 May 1994}

Ms 138SR-122 


\section{APPENDIX}

\section{Tests of the Opal Leach on Selected Site 847 Samples}

In this study, we used a $2 \mathrm{M} \mathrm{Na}_{2} \mathrm{CO}_{3}$ leach of a ground material to quantify the biogenic opal portion of the sample. This leach was made on a dried sample after removal of the carbonate and organic fractions. S. Hovan (U. of Michigan) used a sequential leach to quantify the lithogenic portion of Leg 138 samples. His values were significantly lower than our estimates based on $\left[100-\left(\mathrm{CaCO}_{3}\right.\right.$ + opal)], and he suggested that incomplete digestion of opal with our method was part of the discrepancy. Examination of the residual material after a 6-hr leach in an $85^{\circ} \mathrm{C}$ hot water bath revealed that some samples contained relatively undigested radiolarian parts. To quantify this additional opal material not digested by the primary leach and possibly to resolve the discrepancy noted by Hovan, additional digestions were made using a stronger base $(2 \mathrm{~N} \mathrm{NaOH})$ for $5 \mathrm{hr}$ and then $8 \mathrm{hr}$ more to ensure complete digestion of the opal. The results are presented in the Appendix Table and Appendix Figure.

The duplicate treatments of the standard opal leach were performed on 9 samples of high to low carbonate and opal concentrations (18 total in the test analyşis). The test samples were taken from the remaining one-third of the $5-\mathrm{cm}^{3}$ samples of bulk sediment and were not ground to be consistent with the treatment of samples by Hovan. After $6 \mathrm{hr}$ in the hot water bath, the eight treated samples were centrifuged, $15 \mathrm{~mL}$ of the supernatant was removed for analysis, and then most of the remaining portion of the supernatant was removed without disturbing the solid material on the bottom of the centrifuge tube. These residuals of the leach were rinsed 3 times with hot distilled and deionized water (DDW). After the final rinse, one of the duplicates of each sample was sieved at $38 \mu \mathrm{m}$ to separate the coarse biogenic from fine silt and clay materials. This provided a $<38 \mu \mathrm{m}, \mathrm{a}>38 \mu \mathrm{m}$, and a total portion for each sample (Appendix Table). The unsieved sample was analyzed and compared to the combination of the $<38$ and $>38 \mu \mathrm{m}$ portions to ensure that none of the sample had been lost during the sieving process. All residuals were dried to remove remaining rinse water. Then $17.5 \mathrm{~mL}$ of $2 \mathrm{~N} \mathrm{NaOH}$ was added, and the centrifuge tubes were placed in a hot water bath for $5 \mathrm{hr}$, with stirring at hourly intervals. The tubes then were centrifuged and $15 \mathrm{~mL}$ of the supernatant was removed for analysis. The leach residuals of the split and unsplit portions of the first four samples in Appendix Table were rinsed three times with hot DDW. Again, the residuals of these four samples were dried and treated with $17.5 \mathrm{~mL}$ of $2 \mathrm{~N} \mathrm{NaOH}$. The tubes were placed in a hot water bath for $8 \mathrm{hr}$, centrifuged, and $15 \mathrm{~mL}$ was removed for analysis. The residuals in all 12 tubes were examined under a microscope; no parts of the siliceous organisms were observed.

In all samples, more than $75 \%$ of the total silica leached was removed by the first treatment (Appendix Table and Appendix Figure). Of the additional silica removed with subsequent leaches, most came from the $<38-\mu \mathrm{m}$ fraction. This suggests that radiolarian parts did not contribute to much additional opal. The highest silica values in supernatants using the $\mathrm{NaOH}$ leach are from the $<38-\mu \mathrm{m}$ portion of low-carbonate samples (Appendix Table). We suggest that this silica was leached from clays and was not of biogenic origin. Therefore, although additional silica was leached from all samples, the source of most of this silica probably is the clays, not the biogenic opal. As far as opal from undigested radiolarians is concerned, the contribution is likely to be small. A total of $0.51 \%$ Si was leached from the $>3-\mu \mathrm{m}$ fraction of Sample 138-847C$1 \mathrm{H}-3,92 \mathrm{~cm}$. The original weight of the sample was $42.92 \mathrm{mg}$, and this amount of silicon represents $\sim 0.55 \mathrm{mg}$ of opal $(2.5 \times 0.219 \mathrm{mg} \mathrm{Si}$-opal $=0.548 \mathrm{mg}$ of opal). Takahashi and Honjo (1983) estimated that a typical radiolarian from Quaternary sediments weighed $0.136 \mu \mathrm{g}$. Thus, a total of 4000 radiolarians might account for the silica removed in the 13-hr $\mathrm{NaOH}$ leach of Sample $138-847 \mathrm{C}-1 \mathrm{H}-3,92 \mathrm{~cm}$. Visually, this is a large number, but it constitutes only about $4 \%$ of the total opal in the sample. However, this could be part of the difference between the terrigenous amount estimated from $[100-(\mathrm{CaCO} 3+$ opal)] and the sequential leach of Hovan (this volume). 
Appendix Table

Opal Extraction Tests

\begin{tabular}{|c|c|c|c|c|c|c|c|c|c|c|c|c|}
\hline $\begin{array}{l}\text { Core, section, } \\
\text { interval }(\mathrm{cm})\end{array}$ & $\begin{array}{l}\text { Weight } \\
\text { (mg) }\end{array}$ & $\begin{array}{l}\mathrm{Si}^{\mathrm{a}} \\
(\%)\end{array}$ & $\begin{array}{c}\text { Mean } \\
\mathrm{Si} \\
(\%)\end{array}$ & Split $^{b}$ & $\begin{array}{l}\mathrm{Si}^{\mathrm{e}} \\
(\%)\end{array}$ & $\begin{array}{c}\text { Mean } \\
\mathrm{Si} \\
(\%)\end{array}$ & Split & $\begin{array}{l}\mathrm{Si}^{\mathrm{U}} \\
(\%)\end{array}$ & $\begin{array}{c}\text { Mean } \\
\mathrm{Si} \\
(\%)\end{array}$ & $\begin{array}{l}\mathrm{Si}^{e} \\
(\%)\end{array}$ & $\begin{array}{l}\mathrm{Si}^{\mathrm{f}} \\
(\%)\end{array}$ & $\begin{array}{c}\mathrm{CaCO}_{3 \mathrm{~g}} \\
(\%)\end{array}$ \\
\hline 138-847C-1H-3, 92 & $\begin{array}{l}48.97 \\
42.92\end{array}$ & $\begin{array}{l}11.41 \\
11.97\end{array}$ & $\begin{array}{l}11.41 \\
11.97\end{array}$ & $\begin{array}{l}\text { Total } \\
<38 \mu \mathrm{m} \\
>38 \mu \mathrm{m}\end{array}$ & $\begin{array}{l}1.28 \\
1.24 \\
1.12 \\
1.03 \\
0.11 \\
\text { ND }\end{array}$ & $\begin{array}{l}1.26 \\
1.08 \\
0.11\end{array}$ & $\begin{array}{l}\text { Total } \\
<38 \mu \mathrm{m} \\
>38 \mu \mathrm{m}\end{array}$ & $\begin{array}{l}0.30 \\
0.31 \\
0.12 \\
0.11 \\
0.39 \\
0.41\end{array}$ & $\begin{array}{l}0.31 \\
0.12 \\
0.40\end{array}$ & $\begin{array}{l}12.98 \\
13.68\end{array}$ & 11.83 & 49.85 \\
\hline $138-847 \mathrm{C}-1 \mathrm{H}-4,137$ & $\begin{array}{l}45.34 \\
45.33\end{array}$ & $\begin{array}{l}10.24 \\
10.07\end{array}$ & $\begin{array}{l}10.24 \\
10.07\end{array}$ & $\begin{array}{l}\text { Total } \\
<38 \mu \mathrm{m} \\
>38 \mu \mathrm{m}\end{array}$ & $\begin{array}{l}0.78 \\
0.74 \\
0.53 \\
0.44 \\
0.17 \\
0.11\end{array}$ & $\begin{array}{l}0.76 \\
0.49 \\
0.14\end{array}$ & $\begin{array}{l}\text { Total } \\
<38 \mu \mathrm{m} \\
>38 \mu \mathrm{m}\end{array}$ & $\begin{array}{l}0.08 \\
0.09 \\
0.03 \\
\text { ND } \\
0.00 \\
0.00\end{array}$ & $\begin{array}{l}0.09 \\
0.03 \\
0.00\end{array}$ & $\begin{array}{l}11.09 \\
10.72\end{array}$ & 11.34 & 63.02 \\
\hline $138-847 \mathrm{C}-1 \mathrm{H}-5,2$ & $\begin{array}{l}46.90 \\
49.49\end{array}$ & $\begin{array}{l}6.08 \\
6.02\end{array}$ & $\begin{array}{l}6.08 \\
6.02\end{array}$ & $\begin{array}{l}\text { Total } \\
<38 \mu \mathrm{m} \\
>38 \mu \mathrm{m}\end{array}$ & $\begin{array}{l}0.64 \\
0.54 \\
0.56 \\
0.46 \\
0.18 \\
0.10\end{array}$ & $\begin{array}{l}0.59 \\
0.51 \\
0.14\end{array}$ & $\begin{array}{l}\text { Total } \\
<38 \mu \mathrm{m} \\
>38 \mu \mathrm{m}\end{array}$ & $\begin{array}{l}0.07 \\
0.05 \\
0.04 \\
0.04 \\
0.01 \\
0.00\end{array}$ & $\begin{array}{l}0.06 \\
0.04 \\
0.00\end{array}$ & $\begin{array}{l}6.73 \\
6.71\end{array}$ & 8.80 & 71.66 \\
\hline $138-847 \mathrm{C}-9 \mathrm{H}-3,135$ & $\begin{array}{l}47.50 \\
63.23\end{array}$ & $\begin{array}{l}3.95 \\
3.88\end{array}$ & $\begin{array}{l}3.95 \\
3.88\end{array}$ & $\begin{array}{l}\text { Total } \\
<38 \mu \mathrm{m} \\
>38 \mu \mathrm{m}\end{array}$ & $\begin{array}{l}1.04 \\
0.95 \\
0.63 \\
0.57 \\
0.28 \\
\text { ND }\end{array}$ & $\begin{array}{l}0.99 \\
0.60 \\
0.28\end{array}$ & $\begin{array}{l}\text { Total } \\
<38 \mu \mathrm{m} \\
>38 \mu \mathrm{m}\end{array}$ & $\begin{array}{l}0.15 \\
0.15 \\
0.06 \\
\text { ND } \\
0.00 \\
0.01\end{array}$ & $\begin{array}{l}0.15 \\
0.06 \\
0.00\end{array}$ & $\begin{array}{l}5.09 \\
4.82\end{array}$ & 3.47 & 75.33 \\
\hline 138-847B-1H-3, 17 & $\begin{array}{l}49.30 \\
53.76\end{array}$ & $\begin{array}{l}14.15 \\
14.11 \\
13.65 \\
13.69\end{array}$ & $\begin{array}{l}14.13 \\
13.67\end{array}$ & $\begin{array}{l}\text { Total } \\
<38 \mu \mathrm{m} \\
>38 \mu \mathrm{m}\end{array}$ & $\begin{array}{l}2.02 \\
2.00 \\
1.84 \\
1.84 \\
0.07 \\
0.07\end{array}$ & $\begin{array}{l}2.01 \\
1.84 \\
0.07\end{array}$ & & & & $\begin{array}{l}16.14 \\
15.58\end{array}$ & 14.97 & 28.14 \\
\hline $138-847 \mathrm{C}-6 \mathrm{H}-1,47$ & $\begin{array}{l}50.72 \\
50.66\end{array}$ & $\begin{array}{l}17.52 \\
17.46 \\
17.71 \\
17.81\end{array}$ & $\begin{array}{l}17.49 \\
17.76\end{array}$ & $\begin{array}{l}\text { Total } \\
<38 \mu \mathrm{m} \\
>38 \mu \mathrm{m}\end{array}$ & $\begin{array}{l}3.46 \\
3.46 \\
2.67 \\
2.67 \\
0.81 \\
0.74\end{array}$ & $\begin{array}{l}3.46 \\
2.67 \\
0.78\end{array}$ & & & & $\begin{array}{l}20.95 \\
21.21\end{array}$ & 16.35 & 22.63 \\
\hline $138-847 \mathrm{C}-6 \mathrm{H}-1,62$ & $\begin{array}{l}50.44 \\
50.27\end{array}$ & $\begin{array}{l}14.26 \\
14.30 \\
14.56 \\
14.19\end{array}$ & $\begin{array}{l}14.28 \\
14.38\end{array}$ & $\begin{array}{l}\text { Total } \\
<38 \mu \mathrm{m} \\
>38 \mu \mathrm{m}\end{array}$ & $\begin{array}{l}3.78 \\
3.76 \\
2.93 \\
2.88 \\
0.55 \\
0.55\end{array}$ & $\begin{array}{l}3.77 \\
2.91 \\
0.55\end{array}$ & & & & $\begin{array}{l}18.05 \\
17.83\end{array}$ & 14.17 & 22.75 \\
\hline 138-847D-6H-3, 32 & $\begin{array}{l}50.27 \\
52.88\end{array}$ & $\begin{array}{c}15.48 \\
\text { ND } \\
15.44 \\
15.43\end{array}$ & $\begin{array}{l}15.48 \\
15.44\end{array}$ & $\begin{array}{l}\text { Total } \\
<38 \mu \mathrm{m} \\
>38 \mu \mathrm{m}\end{array}$ & $\begin{array}{l}3.03 \\
3.03 \\
1.97 \\
1.97 \\
0.72 \\
0.72\end{array}$ & $\begin{array}{l}3.03 \\
1.97 \\
0.72\end{array}$ & & & & $\begin{array}{l}18.51 \\
18.13\end{array}$ & 14.80 & 19.28 \\
\hline 138-847D-6H-3, 47 & $\begin{array}{l}50.42 \\
50.65\end{array}$ & $\begin{array}{l}16.02 \\
15.96 \\
17.21 \\
17.23\end{array}$ & $\begin{array}{l}15.99 \\
17.22\end{array}$ & $\begin{array}{l}\text { Total } \\
<38 \mu \mathrm{m} \\
>38 \mu \mathrm{m}\end{array}$ & $\begin{array}{l}3.38 \\
3.38 \\
2.78 \\
2.78 \\
0.62 \\
0.62\end{array}$ & $\begin{array}{l}3.38 \\
2.78 \\
0.62\end{array}$ & & & & $\begin{array}{l}19.37 \\
20.62\end{array}$ & 12.80 & 25.54 \\
\hline
\end{tabular}

"Si concentrations for samples treated with the hot $\mathrm{Na}_{2} \mathrm{CO}_{3}$ leach described in the "Methods" section (this chapter), except that the samples were not ground. All samples were run in duplicate. $\mathrm{ND}=$ no data.

bResiduals after the first leach were rinsed three times with hot DDW, and one of the residuals for each sample was sieved at $38 \mu \mathrm{m}$. Total is the unsplit duplicate.

c $S$ i concentrations for samples treated with $17.5 \mathrm{ml}$ of $2 \mathrm{~N} \mathrm{NaOH}$ and placed in a hot water bath for $5 \mathrm{hr}$.

dSi concentrations for four of the samples and the respective splits that were rinsed, dried, and treated with $17.5 \mathrm{ml}$ of $2 \mathrm{~N} \mathrm{NaOH}$ and placed in a hot water bath for $8 \mathrm{hr}$.

eSum of mean Si concentrations from the sequential extractions for each sample. The sum for samples that were split includes analyses of both the less than and greater than $38-\mu \mathrm{m}$ portions.

'Si concentrations from Table 2

${ }^{-} \mathrm{CaCO}_{3}$ concentrations from Table 2. 
Sample 138-847C $1 \mathrm{H}-3,92 \mathrm{~cm}$

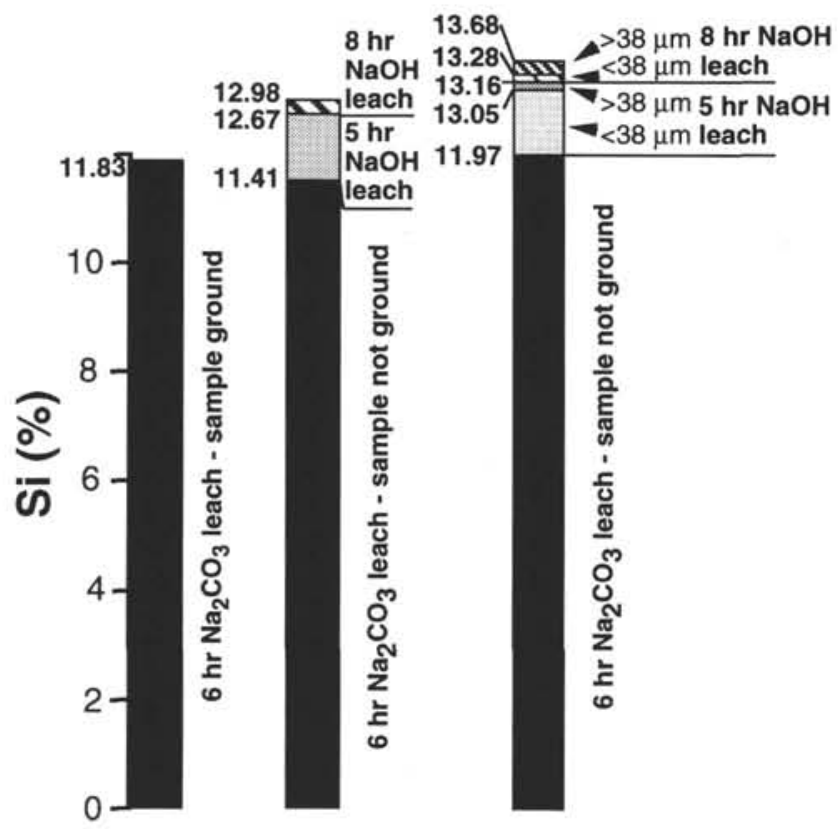

B

Sample

138-847C $1 \mathrm{H}-4,137 \mathrm{~cm}$
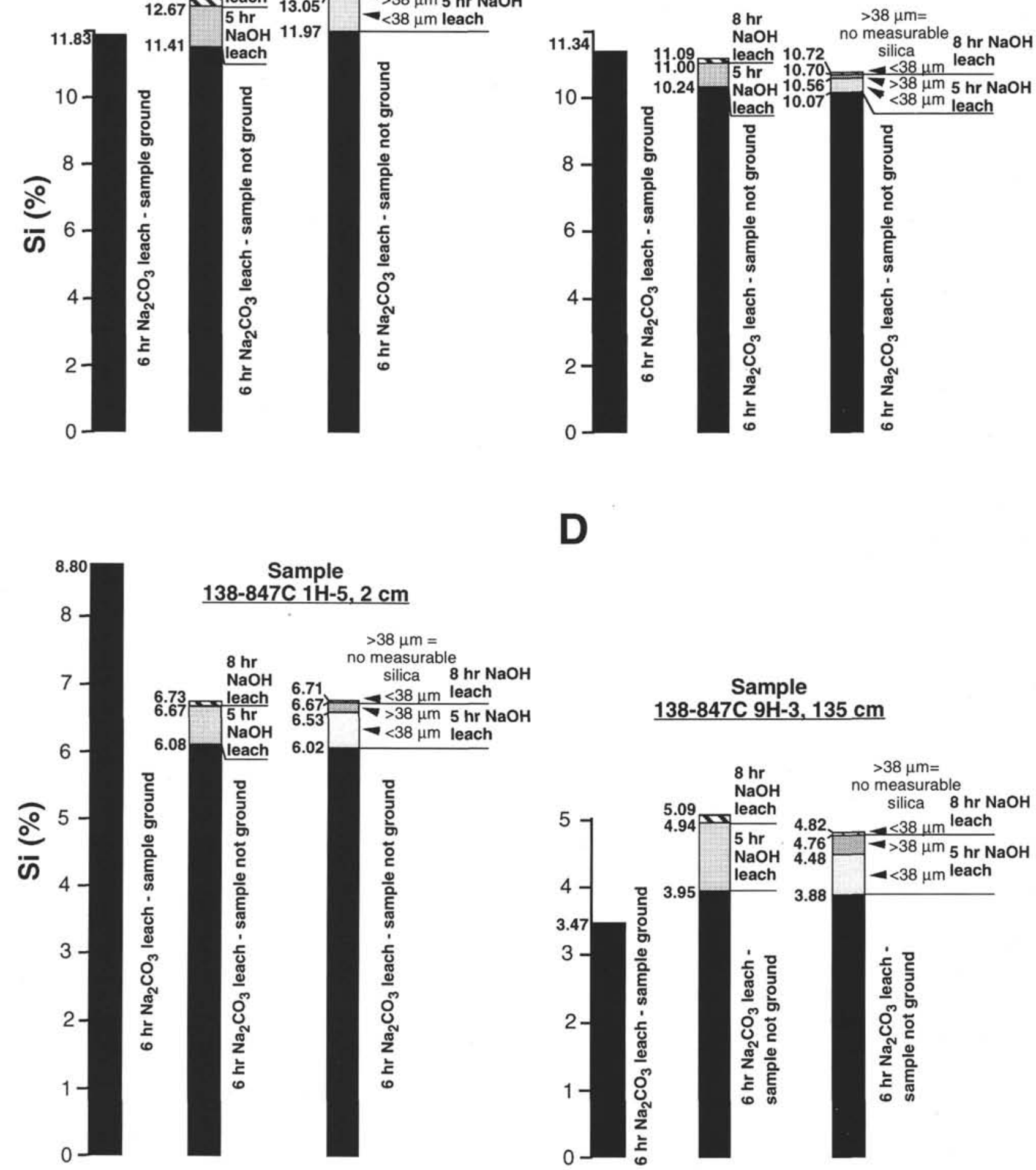

D

Appendix Figure. Stacked histograms of silicon concentrations in test samples (A-D) of the opal leach from carbonate-rich and -poor intervals. Each sample was analyzed in triplicate; one portion was ground and treated using the standard technique. These values are from data listed in Table 2 of this chapter and plotted on the left. Two additional portions were not ground and first treated with a 6-hr $\mathrm{Na}_{2} \mathrm{CO}_{3}$ leach (solid black bars), then were treated with a 5-hr $\mathrm{NaOH}$ leach (dotted patterns), and finally with an 8-hr NaOH leach (stripped patterns). The center plots in all samples are results without sieving the material, whereas the plot on the right is from residuals that were sieved at $38 \mu \mathrm{m}$ after the first $\mathrm{Na}_{2} \mathrm{CO}_{3}$ leach. Most of the additional silicon removed from the solids was from the fine fraction. The large difference in silicon percentages between the ground and unground portions of Sample $138-847 \mathrm{C}-1 \mathrm{H}-5,2 \mathrm{~cm}$, is attributed to sediment inhomogeneities. 